\title{
Novel Semi-Implicit Locally Conservative Galerkin SILCG Solvers: Applications to One Dimensional Human Circulation, Heat Transfer and Ageing Problems
}

\author{
Hasan, Hayder M.
}

How to cite:

Hasan, Hayder M. (2018) Novel Semi-Implicit Locally Conservative Galerkin SILCG Solvers: Applications to One Dimensional Human Circulation, Heat Transfer and Ageing Problems. Doctoral thesis, Swansea University. http://cronfa.swan.ac.uk/Record/cronfa48065

Use policy:

This item is brought to you by Swansea University. Any person downloading material is agreeing to abide by the terms of the repository licence: copies of full text items may be used or reproduced in any format or medium, without prior permission for personal research or study, educational or non-commercial purposes only. The copyright for any work remains with the original author unless otherwise specified. The full-text must not be sold in any format or medium without the formal permission of the copyright holder. Permission for multiple reproductions should be obtained from the original author.

Authors are personally responsible for adhering to copyright and publisher restrictions when uploading content to the repository.

Please link to the metadata record in the Swansea University repository, Cronfa (link given in the citation reference above.) 


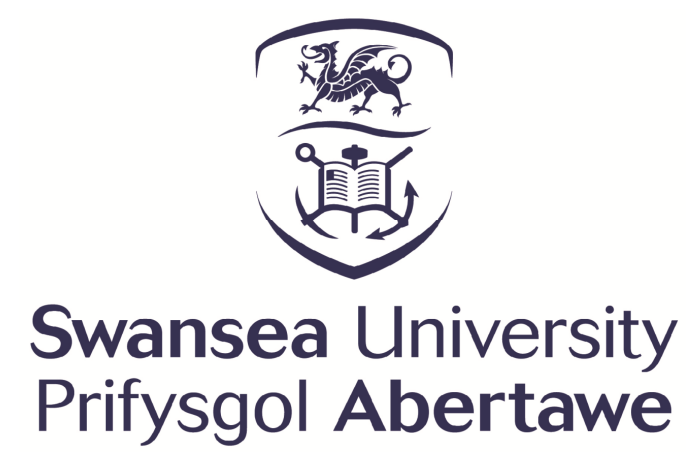

\title{
Novel Semi-Implicit Locally Conservative Galerkin SILCG Solvers: Applications to One Dimensional Human Circulation, Heat Transfer and Ageing Problems
}

\author{
by \\ Hayder Mohammed Hasan
}

Thesis submitted to Swansea University in fulfilment of the requirements for the Degree of Doctor of Philosophy

College of Engineering, Swansea University, UK 2018 


\section{Abstract}

In this work, three novel, locally conservative Galerkin methods in their semi-implicit form are developed for one dimensional blood flow and heat transfer modelling in a human arterial network. The study is also extended for predicting aging effects on the circulation function with an associated thermoregulatory mechanism. These semi-implicit discretizations are the second order Taylor expansion (SILCG-TE) method, the streamline upwind Petrov-Galerkin (SILCG-SUPG) procedure and the backward in time and central in space (SILCG-BTCS) method.

In the well established fully explicit locally conservative Galerkin method (LCG), enforcement of the flux continuity condition at the element interfaces allows solving the discretized system of equations at the element level. For problems with a large number of degrees of freedoms, this offers a significant advantage over the standard continuous Galerkin (CG) procedure. The original explicit LCG method is used for assessing the accuracy of the proposed new methods.

First, mass and momentum equations are solved in the whole system of 91 arterial segments by using the proposed techniques. Results demonstrate that the proposed SILCG methods are stable and as accurate as the explicit LCG method. Among the three methods proposed, the SILCG-BTCS method requires considerably lower number of iterations per element, and thus requires the lowest amount of CPU time. On the other hand, the SILCG-TE and SILCG-SUPG methods are stable and accurate for larger time step sizes due to the presence of the stabilization terms from Taylor expansion based approach and 
streamline upwind Petrov-Galerkin method.

Second, the three approaches are extended to the heat problem by solving the energy equation and combining with blood flow. Here, the interaction between temperature and flexible vessels is tackled. Again, the outcomes show that the new techniques provide desirable accuracy and stability similar to the flow. As SILCG-BTCS is the simplest and fastest one for flow and the same conclusions are derived for heat transfer. Similarly, SLICG-TE and SILCG-SUPG methods still admit higher time steps.

Finally, ageing effect is considered on both flow and heat in a human body. The methods presented previously are adapted by changing the necessary parameters related to ageing. The results obtained confirm the ability of the proposed methods for predicting the changes that occur with age such as the changes in blood pressure, flow and heat transfer. Significant consequences of ageing are highlighted. 


\section{Declaration}

This work has not previously been accepted in substance for any degree and is not being concurrently submitted in candidature for any degree.

Signed (candidate) Date

\section{Statement 1}

This thesis is the result of my own investigations, except where otherwise stated. Other sources are acknowledged by footnotes giving explicit references. A bibliography is appended.

Signed (candidate)

Date

\section{Statement 2}

I hereby give consent for my thesis, if accepted, to be available online in the University's Open Access repository and for inter-library loan, and for the title and summary to be made available to outside organizations.

Signed $\ldots \ldots \ldots \ldots \ldots \ldots \ldots$ (candidate) Date 


\section{Dedication}

To My Family,

for their love, patience and encouragement. 


\section{Acknowledgements}

I would like to express my deepest gratitude to my supervisor Prof. Perumal Nithiarasu for his endless support, encouragement, patience and for sharing the knowledge that has contributed to my research. I am also grateful to my second supervisor Dr. Igor Sazonov.

I would like to thank the Ministry of higher education and scientific research in Iraq and its representative in the London the Iraqi cultural attaché/London for their support and for giving me this opportunity to join the scholarships program. Thanks also go to Shatra Technical Institute and Southern Technical University/Iraq for all support during study time.

My great thanks go to my friend Dr. Alberto Coccarelli for the valuable discussions and sharing knowledge during my study. Also, sincere thanks go to my colleague Mr. Jason Carson for sharing useful information about study aspects. I also would like to thank everyone in College of Engineering at Swansea University for providing all help and facilities making everything run smoothly.

Great thanks go to my father who passed away at beginning of my Ph.D. journey. As I was in a different country, I did not say goodbye to you, here I deeply thank you. My sincere thanks go to my mother who is always my candle that lights up my life during dark times. I also thank my wife Mrs. Huda Saleh Mahdi for all support, help and valuable advice in different aspects. Special thanks go Mr. Hayder Saleh Mahdi for the help and support in my home country. Great thanks go to my brother Azzam for the support and help in the home country. Also, many thanks go to my children, Ali, Fatimah and Hussein for the joy and happiness you have brought to my life. 


\section{Contents}

1 Introduction 1

1.1 Numerical methods for cardiovascular system . . . . . . . . . . 2

1.1.1 Flow modelling of human circulation . . . . . . . . . 2

1.1.2 Modelling of heat transfer in a human body . . . . . . . . . . . 4

1.1.3 Developing an efficient model for ageing . . . . . . . . . 6

1.2 Work objectives . . . . . . . . . . . . . . . . . . 12

2 Modelling of blood flow in human circulatory system 13

2.1 Introduction . . . . . . . . . . . . . . . . . . . 14

2.2 Mathematical formulation of the problem . . . . . . . . . . 15

2.2.1 Governing equations . . . . . . . . . . . . 15

2.2.2 Numerical schemes . . . . . . . . . . . . . . . 16

2.2.3 Solution procedure . . . . . . . . . . . . . 21

2.2.4 Network boundary conditions . . . . . . . . . . . 23

2.3 Results and discussions . . . . . . . . . . . . . . . . . 28

2.3.1 Wave-forms along the network . . . . . . . . . . 30

2.3.2 Spatial convergence ................. 33

2.3.3 Computational efficiency . . . . . . . . . . . . . . 39

2.4 Conclusions . . . . . . . . . . . . . . . . . . . . 39 
3 Modelling energy transport within human body 41

3.1 Introduction . . . . . . . . . . . . . . . . . . . 42

3.2 Mathematical formulations . . . . . . . . . . . . . . . 42

3.2.1 Numerical methods . . . . . . . . . . . . . . . . . . 44

3.2 .2 Solution algorithm $\ldots \ldots \ldots 4 \ldots$

3.2 .3 Solid discretization . . . . . . . . . . . . . . . . 47

3.2.4 Initial and bondary conditions . . . . . . . . . . . . . 48

3.2.5 Arterial tree-solid tissues architecture . . . . . . . . . . . . 49

3.2 .6 Thermoregulatory System _ . . . . . . . . . . . . . . 51

3.2.7 Coupling blood with solid systems . . . . . . . . . . . . . 54

3.3 Results and discussion $\ldots \ldots \ldots \ldots \ldots$

3.4 Conclusions . . . . . . . . . . . . . . . . . 61

4 Modelling ageing effects

4.1 Introduction . . . . . . . . . . . . . . . . . . 70

4.2 Modelling ageing effects on the human body . . . . . . . . . . . 70

$4.2 .1 \quad$ Arterial system . . . . . . . . . . . . . . 70

4.2 .2 Solid system . . . . . . . . . . . . . . 76

4.2.3 Regulatory system .................. 79

4.3 Results and discussion $\ldots \ldots \ldots \ldots$. . . . . . . . . . . . 84

4.3.1 Human circulation results . . . . . . . . . . . . 85

4.3.2 Tissues heat transfer results f . . . . . . . . . . . 87

4.4 Conclusions . . . . . . . . . . . . . . . . . . . . . . 89

5 Conclusions and future works $\quad 93$

5.1 Introduction . . . . . . . . . . . . . . . . . . . . . . . 94

5.2 Conclusions . . . . . . . . . . . . . . . . . . . . 94

5.3 Future investigations . . . . . . . . . . . . . 96 


\section{List of Figures}

2.1 One-dimensional elements in CG (A) and LCG methods (B). . . . . . . 20

2.2 Input pressure wave. . . . . . . . . . . . . . . . . . 24

2.3 A typical arterial segment with inlet and outlet boundaries. . . . . . . . 26

2.4 Different types of arterial tree discontinuities. . . . . . . . . . . . . 27

2.5 Systemic circulation model. . . . . . . . . . . . . . . . . . . . . . 29

2.6 Pressure variation in different arterial segments. . . . . . . . . . . 31

2.7 Flow variation in various segments. . . . . . . . . . . . . . 32

2.8 Number of iterations to number of elements ratio for for all three approaches. Jacobian matrix is calculated at every iteration. . . . . . . . 35

2.9 Number of iterations to number of elements ratio for for all three approaches. Jacobian matrix is calculated at every time step. . . . . . . . 36

2.10 Mesh convergence of SILCG-TE method. . . . . . . . . . . . . . . . 37

2.11 Pressure and flow variations in the ascending aorta obtained by the new methods against the original LCG using a fine mesh. . . . . . . . . . 38

3.1 Schematic representation of arterial segment. . . . . . . . . . . 43

3.2 Manikin configuration of main arterial network with tissues (14 cylinders) in human body. . . . . . . . . . . . . . . . . . . . 50

3.3 Coupling of an artery with tissue (represented by cylinders) . . . . . . 56 
3.4 Tympanic, rectal temperatures and heat losses at different external condi-

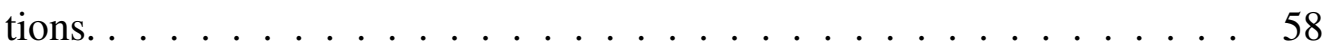

3.5 Core and skin temperature variations in a bare body under hot exposure. . 59

3.6 Core temperature variations in a bare body under cold exposure. . . . . . 60

3.7 Blood temperature distributions in three locations and under various outside conditions. . . . . . . . . . . . . . . . . 63

3.8 Number of iterations to number of elements ratio for for all three approaches. Jacobian matrix is calculated at every iteration, temperature loop is considered only. . . . . . . . . . . . . . . . . . . . . 64

3.9 Number of iterations to number of elements ratio for for all three approaches. Jacobian matrix is calculated at every iteration, total cost is estimated as iterations/no. of elements ratio of flow+the one from the temperature loop shown in previous figure. . . . . . . . . . . . . 65

3.10 Number of iterations to number of elements ratio for for all three approaches. Jacobian matrix is calculated at every time step (not every iteration), total cost is estimated as iterations/no. of elements ratio of flow+the one from the temperature loop. . . . . . . . . . . . . . 66

3.11 Blood velocity variation in various locations along the arterial tree. . . . 67

3.12 Vessel cross-section area variation in different locations along the arterial

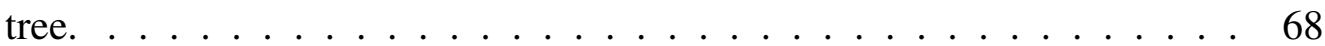

4.1 Input pressure wave for different ages. . . . . . . . . . . . . . . 76

4.2 Elastance curve for different ages. . . . . . . . . . . . . . 85

4.3 Pressure waveforms at ascending aorta artery for different ages. Reference data are taken from $[1] . \ldots \ldots$. . . . . . . . . . . . 87

4.4 Pressure waveforms at brachial artery for different ages. Reference data are taken from $[1] . \ldots \ldots \ldots \ldots$ 
4.5 Systolic and diastolic pressure at ascending aorta and brachial artery for different ages. Experimental measurements are taken from [2, 3]. . . . . . 89

4.6 Pressure propagation indicators for different ages. Experimental measuements are taken from $[3] \ldots \ldots$. . . . . . . . . . . . . . . . 90

4.7 Core temperature evolution in time for different modelling assumptions. Literature results are taken from [4] (core temperature is assumed to be $37.3{ }^{\circ} \mathrm{C}$ at the beginning of the test). . . . . . . . . . . . 91

4.8 Mean skin temperature evolution in time for different modelling assumptions. Experimental results are taken from [5] (skin temperature is assumed to be $34.4{ }^{\circ} \mathrm{C}$ at the beginning of the test). . . . . . . . . . . 92 


\section{List of Tables}

2.1 Time steps considered and related CFL, $l_{e}=0.25 \mathrm{~cm} \ldots \ldots \ldots 31$

2.2 Percentage $R M S E_{p}$ and $R M S E_{F}$ for all cases shown in Figures 2.6 and 2.7. 34

2.3 Averaged percentage $R M S E_{p}$ and $R M S E_{F}$ for all values shown in Table 2.2 for each method. . . . . . . . . . . . . . . . . . 37

2.4 Computational speed CPU time per cardiac cycle, $l_{e}=0.25 \mathrm{~cm}$. . . . . 39

3.1 Tissue physical properties $[6] \ldots \ldots \ldots \ldots$. . . . . . . . . 51

3.2 Computational speed CPU time per cardiac cycle, $l_{e}=0.25 \mathrm{~cm}$. . . . 61

4.1 Age-dependent fitting coefficients of Eq.(4.2), taken from [1]. . . . . . . 71

4.2 Set of equations used for modelling terminals variations. . . . . . . . . 73

4.3 LV reference data for each age decade. Values for $p_{E D}$ were obtained by readapting the age-relationship extrapolated from [7] to a reference value $4.5 \mathrm{mmHg}$ at $35 \mathrm{yr} . \ldots \ldots \ldots 77 \ldots \ldots \ldots$

4.4 Set of extrapolated equations used for modelling tissue variations. . . . . 79

4.5 Body parts sweating coefficients. Such parameters are obtained by readapating the values from [4] for the current body solid architecture. The coefficients for the arm/forearm and the thig/leg are obtained dividing respectively, the sum of the coefficients for arms and hands, and the sum of coefficients for legs and feet by 4. . . . . . . . . . . . . . . . 80

4.6 Decline of the thermal sensitivity $\Delta T_{\text {skin,dec }}$ and $\Delta T_{\text {core,dec }}$ with age, from [4]. 81 
4.7 Body parts cutaneous perfusion coefficients. Such parameters are obtained by re-adapating the values from [8] for the current body solid architecture. It is important to note that the coefficients for the neck and shoulder are assumed to be equal respectively to the coefficents for the

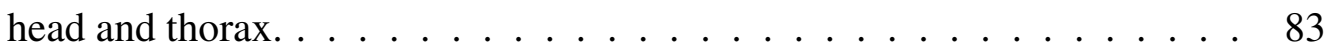

4.8 Body parts sweating coefficients. These parameters are obtained by readapting the values from [9] for the current body solid architecture. Head and shoulders include, respectively, the face and the back. . . . . . . . . . 84 


\section{Nomenclature}

\section{Latin letters}

A

$A_{0}$

$A_{\text {ext }}$

$A_{\text {in }}$

C

$\mathrm{CO}$

$c_{\max }$

$c_{P}$

$\mathbf{D}_{e T}$

E

$\mathbf{F}$

F

f

$f_{c l}$

$\mathbf{f}_{\Gamma e}$

H

$h$

HR

$h_{\text {ext }}$
Vessel cross-sectional area

Vessel stress free cross-sectional area

External surface area

Internal surface area

Convection matrix

Cardiac output

Maximum intrinsic wave speed

Specific heat at constant pressure of fluid

Element matrix for temperature diffusion in fluid

Young's modulus

Convective and Taylor Galerkin fluxes

flow

External force vector

Clothing surface factor

Boundary terms at element boundaries

Matrix of convection term

Tube wall thickness

Heart Rate

External heat transfer coefficient 


\begin{tabular}{|c|c|}
\hline$h_{\text {in }}$ & Internal heat transfer coefficient \\
\hline$h_{\text {rad }}$ & Radiative heat transfer coefficient \\
\hline$G$ & Flux for energy conservation equation \\
\hline I & Identity matrix \\
\hline $\mathbf{J}$ & Jacobian matrix \\
\hline $\mathbf{K}_{e}$ & $\begin{array}{l}\text { Element matrix for convection and Taylor Galerkin } \\
\text { terms }\end{array}$ \\
\hline $\mathbf{K}_{e T}$ & $\begin{array}{l}\text { Element matrix for temperature convection and Tay- } \\
\text { lor Galerkin terms }\end{array}$ \\
\hline$k_{t}$ & Wall thermal conductivity \\
\hline$L$ & Tube length \\
\hline $\mathbf{L}_{e}$ & Element matrix of source terms \\
\hline $\mathbf{L}_{e T}$ & Element matrix of temperature source terms \\
\hline $\mathbf{L}$ & Eigenmatrix \\
\hline$l_{e}$ & Element length \\
\hline $\mathbf{M}$ & Mass matrix \\
\hline $\mathbf{M}_{e}$ & Element mass matrix \\
\hline $\mathbf{M}_{e T}$ & Element mass matrix for temperature \\
\hline$\dot{m}_{\text {skin }}$ & Skin perfusion flow \\
\hline$\dot{m}_{\text {swea }}$ & Sweating flow \\
\hline $\mathbf{N}$ & Shape function vector \\
\hline$N$ & Shape function \\
\hline$p$ & Pressure \\
\hline$p_{\text {ext }}$ & External pressure \\
\hline$p_{\text {in }}$ & Input pressure value \\
\hline$p_{\text {init }}$ & Initial input pressure \\
\hline$p_{\text {out }}$ & Ambient water vapour pressure \\
\hline
\end{tabular}




\begin{tabular}{|c|c|}
\hline$p_{\text {skin }}$ & Skin water vapour pressure \\
\hline$Q$ & Flow rate \\
\hline$Q_{\text {shiv }}$ & Shivering power \\
\hline$q_{b r e}$ & Breathing heat flux \\
\hline$q_{\text {con }, \text { out }}$ & Convective heat flux \\
\hline$q_{m}$ & Metabolic heat production \\
\hline$q_{\text {rad }}$ & Radiative heat flux \\
\hline$q_{\text {swe }}$ & Evaporative heat flux \\
\hline $\mathbf{R}$ & Residual \\
\hline$R_{A V}$ & Aortic valve transmission coefficient \\
\hline$R_{R}$ & Arterial reflection coefficient \\
\hline$R_{\text {swe }, c l}$ & Clothing sweating resistance \\
\hline$r$ & Radial coordinate \\
\hline r.h. & Relative humidity \\
\hline$r_{e x t}$ & Vessel external radius \\
\hline$r_{i n}$ & Vessel internal radius \\
\hline $\mathbf{S}$ & Source vector \\
\hline$T$ & Fluid temperature \\
\hline$T_{\text {core }}$ & Core temperature \\
\hline$T_{\text {out }}$ & Outside environment temperature \\
\hline$T_{\text {shiv }}$ & Shivering temperature threshold \\
\hline$T_{\text {skin }}$ & Mean skin temperature \\
\hline$T_{\text {swe }}$ & Sweating temperature threshold \\
\hline$T_{t}$ & Tissue temperature \\
\hline$T_{t, i n}$ & Inner wall temperature \\
\hline $\mathbf{U}$ & Primitive variable vector of area and \\
\hline
\end{tabular}


$\tilde{\mathbf{U}}$

$u$

$u_{\text {in }}$

$\dot{W}_{\text {shear }}$

$\dot{W}_{\text {wall }}$

$w_{f}, w_{b}, w_{t e m p}$

$w_{\text {skin }}$

$x$

\section{Greek letters}

$\alpha$

$\alpha_{f}$

$\beta$

$\Delta t$

$\theta$

$\Lambda$

$\lambda_{f}, \lambda_{b}$

$\lambda_{\text {temp }}$

$\mu$

$v_{\text {air }}$

$\xi$

$\rho$

$\rho_{t}$

$\sigma$

$\dot{\Phi}_{\text {cond }}$

$\dot{\Phi}_{\text {conv }}$
Approximated primitive variable vector of area and velocity

Fluid velocity

Inlet velocity

Loss due to viscous effect

Work by wall forces

Characteristic variables

Skin wetness

Current coordinates

An optimal value for Petrov-Galerkin method Fluid thermal diffusivity

Vessel elastic parameter

Time step

Time integration parameters

Eigenvalues

Forward and backward characteristic speeds

Characteristic speed for temperature

Fluid dynamic viscosity

Air velocity

Ageing parameter

Fluid density

Tissue density

Poisson's ratio

Heat diffusion flux

Heat convection flux 
Perfusion rate

$\Omega$

Reference spatial domain 


\section{Abbreviations}

AV

CFL

LCG

LV

RMSE

SILCG

SILCG-BTCS

SILCG-SUPG

SILCG-TE
Aortic valve

Courant Friedrichs Lewy condition

Locally conservative Galerkin method

Left ventricle

Root mean square relative error

Semi-implicit locally conservative Galerkin method Semi-implicit locally conservative Galerkin method following to backward in time and central in space discrete form

Semi-implicit locally conservative Galerkin method depending on streamline upwind Petrov-Galerkin technique

Semi-implicit locally conservative Galerkin method based on Taylor expansion approach 
Chapter 1

\section{Introduction}




\subsection{Numerical methods for cardiovascular system}

Modelling of various physical aspects in elastic tubes and within cardiovascular system has become an attractive field due to the impacts on human health. Cardiovascular modelling is challenging due to the complexity of the computational domain such as branch locations and deformable nature of the blood vessels. These difficulties are reflected in solving continuity and Navier-Stokes equations through the entire domain with appropriate initial and boundary conditions. Moreover, the predicted velocity impacts energy transport characteristics. So, developing accurate numerical approaches is vital for better understanding of flow and heat transfer characteristics in a circulatory system.

There are several numerical methods available in the literature. Three dimensional approaches have been used when more information is required in specific locations. On the other hand, one dimensional simulations have been widely used to capture true physiological phenomenon. As the computational cost is reasonable, these models have been the interest of many researchers. A brief overview of the previous works is given below.

\subsubsection{Flow modelling of human circulation}

One dimensional flow modelling is a popular approach for computing arterial flow in the human systemic circulation $[10,11]$. These methods represent a very useful tool for studying clinical problems, such as detection of aneurysms $[12,13,14,15,16]$, arterial stenosis $[17,18]$, fractional flow reserve $[19,20]$ and other problems of societal and practical relevance like modelling of heat transfer in a human body [6]. In the last three decades a large number of one dimensional models has been proposed for predicting the blood pressure and flow along the expanded arterial trees. In some specific cases with simplified assumptions such as axisymmetric velocity profiles, the solution has been calculated in an analytical way by means of a Fourier technique [21]. This method has been adopted in other studies [22, 23, 24], and has also been combined with one di- 
mensional electrical analogy [25]. However, such approaches have not accounted for non-linear effects $[26,27,28]$. The non-linear governing equations have been solved using the Finite Difference method [29, 30, 31], system of characteristics [32], Galerkin Least Squares [33], Discontinuous Galerkin methods [34, 35, 28] and Finite Volume methods $[36,37]$. Recently, much effort has been also made to analyze blood flow in anatomically very detailed networks [38]. With regard to the numerical strategy, there is no clear evidence exists in the literature on the most accurate and the fastest suitable method for dealing with one dimensional blood flow modelling. The algorithms available in the literature have also been generally classified according to the time integration scheme adopted. In a fully explicit scheme, the time step must be selected in such a way that the CFL condition is satisfied. This is also the case for prescribing the required boundary conditions $[39,12]$. It was demonstrated in [40] that some explicit methods may represent, in terms of accuracy, a valuable alternative to implicit schemes. Implicit methods have become very popular as they do not have a restriction on the time step. However, the accuracy of the transient solution may be compromised if a significantly larger time step than the one allowed by Courant Friedrichs Lewy CFL condition is combined with a low order time discretization. CFL number is given as [40]

$$
\mathrm{CFL}=\frac{\lambda \Delta t}{\Omega_{e}},
$$

where $\lambda$ is the maximum absolute velocity, $\Delta t$ and $\Omega_{e}$ are respectively the time and spatial steps. In implicit methods, difficulties related to their implementation may arise, due to the high non-linearity of the governing equations. The design of the iterative numerical procedure for computing the solution is crucial, as it may lead to inefficient schemes [41]. In addition, with an implicit method, a realistic representation of the complex boundary conditions encountered in systemic circulation problems is often difficult. For example, the use of the system of characteristics (Riemann method) for prescribing the boundary conditions may be computationally expensive as unconditional stability may be compromised. The alternative is to include such boundary conditions into the implicit formulation 
(matrix) along with the main variables. This, however, may add extra costs to the solution process. Some authors have developed mixed time discretization methods between explicit and implicit schemes in which the main domain has been solved explicitly whereas the bifurcation points have been treated implicitly to ensure a strong coupling of the solution $[42,43]$. Another valuable method has been proposed in [44], where the domain decomposition does not impose any stability restriction on the time step size. In large scale problems, implicit schemes lead to the assembly of large global matrices, leading to potential memory storage issues and reduction of computational efficiency. This may also be associated with the poor convergence of Newton method for systems with a large number of degrees of freedoms [45]. Such issue has been the driving motivation behind the development of quasi-Newton methods, such as Broyden's method. For avoiding an excessive computational cost, some of these methods approximate the Jacobian matrix of the system by means of the finite difference method.

\subsubsection{Modelling of heat transfer in a human body}

Heat transfer in a human body is an essential consideration for studying a variety of circumstances, such as human thermal comfort inside a building [46]. The temperature controlled surgeries is another important field that has been utilized for a long time in cryosurgery for cancer treatment $[47,48,49]$. It is known that developing a robust thermal model may help to understand heat-induced illnesses in various exposure circumstances. So, many models have been developed for predicting heat transfer characteristics inside a human body. The simplified approaches developed in the past treat the human body as a single point with temperature changes permitted in time only. However, three dimensional models predict temperature changes in a human body with better accuracy but it would be expensive [6]. Between different methods, segmental models can be a reasonable choice which accounts for different body layers and shows a good understanding of heat transfer in a human body [6]. The thermal interaction between a human body and 
the environment involves two mechanisms, i) simultaneous heat transfer including convection, radiation, evaporation, conduction and respiration, ii) body self-regulation such as vasodilation, vasoconstriction, shivering and sweating [50].

One of the early works shown by [51] where a two-node approach was considered. As the name suggests, it calculates core and skin temperatures depending on the nodes. This is a good method over the existing lumped ones but it performs poorly for obtaining detailed temperatures distribution. Therefore, a multi-nodes approach introduced by [52, $53,54,55]$ to overcome drawbacks of the previous one. Here, the body is simulated as several cylinders representing arms, hands, legs, feet and trunk. Obviously, this is a sophisticated model but the main restriction is that the blood is assumed to be isothermal. Body division into cylinders is the main attractive approach due to the simplicity and reasonable accuracy $[9,56]$. The cylinders also consist of multilayers representing bone, muscle, viscera, fat, skin, brain and lung. The choice of these layers depends on what part of the body is represented by a cylinder. The thermoregulatory system is also established for achieving better representation of the bioheat transfer term. Others like [57] developed more accurate models by including more details such clothes. However, the major issue of previous works is they do not account for the convective heat that occurs between blood and artery walls. The weak points of most of the previous works are the improper convection between tissue and blood vessels, and the ignoring the pulsatile velocity field which comes as a result of heart function. So, the works developed by $[6,58]$ represent an improvement over the existing works by considering blood effect on heat transfer in the body in addition to the segmented approach.

With respect to the numerical methods, bioheat problem has similar difficulties that previously rose in the case of flow. So, the complexity of the arterial network and the related boundary conditions at the inlet, outlet and junctions is the same here along with discretization procedure for the governing equations. Therefore, we attempt to develop three algorithms that are able to resolve some numerical difficulties that usually arise with 
the iterative Newton solver for linear and non-linear problems like for instance the large matrices and slow convergence. This will be detailed later.

\subsubsection{Developing an efficient model for ageing}

For the elderly human population, sudden environmental changes may represent a serious threat to their lives and thus requires taking immediate and appropriate actions [59]. This is due to the fact that ageing lowers thermal resistance and leads to a less efficient thermoregulatory system. Ageing is a process that can be defined as an intrinsic deterioration of the homoeostatic capabilities of an organism, leading to a constantly increasing risk of death [60]. The accumulation of defective mithocondria represents one of the most accredited factors causing ageing $[61,62,63,64]$. This is because the free radicals produced in oxidation reactions are able to damage several kinds of biologically relevant components such as mitochondrial DNA, lipids, and proteins. Mitochondrial DNA mutations may affect tremendously the cellular survival since this DNA produces enzymes necessary for the oxidative phosphorylation. At the tissue level, such irreversible cellular degradation leads to changes in the organs functionalities, tissues physical properties and volumes. Ageing effects on the human body may also be influenced by the lifestyle and the environmental conditions which the individuals are exposed to. Due to a less efficient immune system, elderly individuals are more vulnerable to pathogens (A pathogen or infectious agent is a biological agent that causes disease or illness to its host) than young adults. Furthermore, ageing affects also the capacity of the body to react to changes in either internal or external conditions, such as physical workload, food digestion, and thermal stress exposure. The energy balance of the body depends on different physiological and anatomical components such as cardiac function, tissue volumes and thermal properties, metabolic production, and may be extremely sensitive to any of these factors variation. Elderly people generally present significant alterations of such components with respect to the younger individuals, which may involve completely different thermal 
responses for the same external conditions. The cardiovascular system undergoes different changes with age, leading to a significant increase in pressure amplitude along the arterial tree. The pulsating nature of arterial blood load originates fatigue and fracture processes of elastin lamellae in large elastic arteries, causing a progressive vessel wall stiffening and a consequent dilation with age. Such decrease in elasticity is associated to an increase in pulsatile wave velocity, and thus for a stiffer arterial tube network reflections return back earlier to the heart. This reduction in compliance is especially valid for large elastic arteries [65, 66]. The study was done by [67] also highlighted the modulation role of ageing especially in arteries belonging to the aortic cross-sectional area. Although the link between ageing and cardiac function adaptation needs still further elucidations, it is widely accepted that the cardiac compensation of such extra load occurs without a significant increase in ventricular wall stress but with a left ventricle wall thickening, which can be associated to a form of concentric hypertrophy [68]. This leads, with age, to an aortic systolic pressure rise and a diastolic pressure fall. For a more exhaustive description of the ageing vascular changes see reference works $[69,70,71]$. These changes in the arterial flow may lead to a different heat exchange by convection with the surrounding tissues. Ageing has also profound effects on the functional and structural properties of the tissues. A valuable study given by [72] provided a key contribution to the topic, by formulating a set of generalized equations for calculating body density with age. However, for modelling purposes, specific studies on tissue components allow characterizing more accurately such ageing effects. The most significant tissue changes are reported in the following. Body tissues are subjected to gradual age-related changes in volume. Although these volume variations are generally non-linear with age, the lack of experimental data makes generally necessary describing such relationships via linear regression techniques. With advancing age the muscle mass starts significant reduction, along with a decrease in elasticity, strength, and functionality. Ageing may also lead to sarcopenia which is defined as a progressive decline in skeletal muscle mass primarily due to age- 
ing, but it may have others causes such as muscle disuse, inadequate nutrition, cachexia (loss of weight, muscle atrophy, fatigue, weakness and significant loss of appetite) [73]. The study of [74] reported some descriptive anthropometric data for elderly people and found out that the decline in body mass index (BMI) paralleled the progressive weight reduction. Older people (age $>50 \mathrm{yr}$ ) generally present subcutaneous fat reduction, which is generally compensated by a slight increase in visceral fat. Across the lifespan, also the bone tissue microstructure is subjected to several changes, some of which may lead to disease conditions, such as osteoporosis. As reported in [75], bone density variations with age depend strongly on the gender. Both cortical and cancellous components contribute to differences in age-related changes in bone tissue $[76,77]$. Others like [78] reported, for both genders, the density variation with age of a mid-thoracic vertebral trabecular bone. It is reported that such density decline occurring for the trabecular component is much higher than the decrease occurring for the global bone. Ageing also affects the basal metabolic rate (BMR), which is defined as the energy requested by the body for guaranteeing homeostasis at rest under thermoneutrality conditions and fasting. As reported in [79], this rate tends to decline at older ages. From a case study of 300 healthy men, the study of [80] concluded a curvilinear relationship between the rest metabolic rate (RMR) and age. Also, [81] drew inferences from data sets of 2 different studies (Oxford $\mathrm{BMR}$ and $\mathrm{FAO} / \mathrm{WHO} / \mathrm{UNU} \mathrm{BMR}$ ) reported in [82] for 2 different weight categories (65 kg and $75 \mathrm{~kg}$ ). For the group $75 \mathrm{~kg}$, both studies showed a similar decrease in BMR with age at an almost constant rate $\approx-0.38 \mathrm{~W} / \mathrm{yr}$. However, these reduction rates extrapolated are only indicative of such approach does not account for age-related body weight changes. It is indeed widely accepted that body weight increases with age till reaching a plateau during middle age, thereafter a gradual reduction occurs, which is inline with tissue thinning. For healthy older people, the body core temperature under thermoneutral conditions does not significantly differ from the one in younger people. An investigation carried out by [83] reported that, with respect to young adults, elderly people under heat 
stress conditions typically respond with attenuated sweating and decreased vasoconstriction. This is accompanied by reduced cardiac outputs and smaller redistributions of blood flow from the splanchnic and renal circulations. Ageing affects also the response to cold stress conditions, involving a reduced peripheral vasoconstriction and shivering energy production. The difference in thermal response between different age groups becomes obviously more evident whether the environmental exposure is extreme, like in case of heat waves or hypothermic conditions. However, due to their limited sensory perception, elderly people are at risk also when they are exposed to mild environmental conditions for long times. When the subject is exposed to a hot stress environment, the body temperature increase is contrasted by the action of the thermoregulatory system, which tries to keep the core temperature within the thermoneutrality range $\left(\sim 36.8-37.5^{\circ} C\right)$ by using different mechanisms such as sweating and vasodilation. If the regulatory mechanisms are not able to level off the thermal energy income with the cooling losses, the thermal balance remains impaired, causing a further temperature rise. Every time that the body core temperature is out of the thermoneutrality range, cellular physiological processes start gradually to deteriorate, and impair organs and regulatory functions. If this situation is prolonged, the person may experience, heat exhaustion first, and then heatstroke which is considered a life-threatening illness. This case is rare for young adults but it is more likely to happen for elderly people because their thermal resistance may be much lower and thus such processes may occur much faster without letting the person to take appropriate countermeasures. The prediction of the temperature distribution in an aged body under thermal stress may, therefore, provide insightful indications for preventing the onset of such pathological conditions. Furthermore, modelling heat transfer in the aged human body may also be useful for other applications, such as temperature controlled surgeries, and ageing related diseases, such as Alzheimer.

For studying heat transport in the human body, several different bioheat transfer models were proposed in the recent past $[84,56,85,86,87,88,6]$, ranging from simple 
lumped models to more complex realistic 3-D representations of the body. Although most of these works present very comprehensive modelling methodologies, ageing effects on the body energy balance are not generally taken into account. A limited number of works were carried out on this topic. A significant contribution made by [81], which modelled ageing effects on the body by modifying parameters such as metabolic rate, cardiac output, body weight, height and body surface area. Age modelling modifications were made according to experimental observations such as [89, 90, 91, 92, 82]. In order to account for ageing factors, the necessary parameters have to be modified [93], the regulated blood perfusion coefficients at the skin, the metabolic rate during hot/cold conditions and decreased the cardiac output. The predicted results showed a good agreement with the experimental results but the model was not validated for extreme thermal stress situations. Another work done by [4] proposed to analyze the core temperature, average skin temperature and skin mass evaporation for a body subjected to hot external conditions $\left(>40^{\circ} \mathrm{C}\right)$. In such work, no significant age-variations in cardiac output and tissues volumes were assumed, but sweating losses depending on age were included. Also, in this case, the predicted results agreed well with the experimental data obtained for two age categories. With regard to the ageing effects on the arterial blood flow, relevant contributions were given by the works of [7] and [1]. In these works the left ventricle thickening, and consequently the heart contractility alternate due to increased arterial stiffening with age, which can be seen as bigger flow resistance. The modelling of such components was carried out according to experimental data and the global methodology was proved to give excellent simulating results against measurements. Another relevant study was carried out by [94], which proposed a modelling strategy describing aortic stiffening and remodelling compensation during ageing. Others like [95] provided a valuable contribution to vascular ageing by proposing for the human aorta a new strain energy function accounting for ageing. It appears that there is still room for improving ageing problem modelling by including more parameters like the effect of pulsating blood flow and heat 
transfer within blood on the circulatory system in elderly individuals. Thus, the current work introduces some numerical model improvements for solving flow and heat transport at the beginning, and then ageing influence is investigated.

In chapters 2 and 3, we propose a set of three new semi-implicit approaches that simplify the numerical procedures without degrading the solution accuracy [10]. These semi-implicit, locally conservative Galerkin (SILCG) methods limit the maximum matrix sizes to the element matrix size. The iterative numerical procedures are considerably simplified. The three versions introduced here are the second order Taylor expansion based method (SILCG-TE), the streamline upwind Petrov-Galerkin (SILCG-SUPG) scheme and the backward in time and central in space (SILCG-BTCS) method. SILCG in its all versions uses only element matrix in the numerical formulation, the resulting system size, if linear elements are employed, are $4 \times 4$ for flow and $2 \times 2$ for heat transfer problems. This small matrix size allows us to easily compute the exact Jacobian matrix every time step. This avoids us the need to use any modified Newton based methods like quasi Newton solvers. This resolves Newton slow convergence that comes as a results of large matrices. The use of element matrices also allows us to increase the mesh size (number of elements) without enlarging the matrix size, so the new methods will require less computer memory demands in comparison with classical Newton method. They also allow us to relax time step restriction that is associated with the fully explicit LCG because the stability condition (CFL number) that is given in Eq. (1.1). All these goals will be discussed in details with relevant results in next two chapters. After that, chapter 4 is dedicated to develop a new model for ageing problem that particularly focuses on elderly population by including some findings available in the literature with the flow and heat transfer models developed in the previous chapters. The aim is to gain more realistic presentation of ageing consequences on blood flow and heat transfer characteristics that will help us to better understand of the problem. A better presentation of the ageing could save individual lives. The number of deaths associated with heatstroke in elderly population is 
very high. Thus, study of the ageing and the relevant consequences on the flow and heat transfer characteristics is subject of interest for many researchers.

\subsection{Work objectives}

The contributions of the present work can be summarized as (a) highlighting an introduction to the SILCG method in three versions for systemic blood circulation, i.e. SILCGTE, SILCG-SUPG, and SILCG-BTCS. (b) Demonstration of its simplicity and accuracy against the original fully explicit LCG method given in [12, 39]. (c) Then, the new three approaches that have been implemented for flow are extended to bioheat transfer problem. Here, the outcomes are compared against the numerical and experimental works developed in $[6,52,84,87,96]$. Finally, SILCG-TE is used to model ageing problem in a human body. The results are validated against $[1,2,3,4,5]$. 


\section{Chapter 2}

Modelling of blood flow in human circulatory system 


\subsection{Introduction}

In this chapter, we propose a family of new semi-implicit approaches that are able to simplify the numerical procedure without compromising the solution accuracy [10]. These semi-implicit locally conservative Galerkin SILCG methods limit the maximum matrix sizes to the element matrix sizes. With the introduction of LCG discretization, the iterative numerical procedure is substantially simplified. The three versions introduced here are the second order Taylor expansion based SILCG-TE method, the streamline upwind Petrov-Galerkin SILCG-SUPG scheme and the backward in time and central in space SILCG-BTCS method. Since SILCG uses only element matrix in the formulation, the resulting system size, if linear elements are employed, is $4 \mathrm{x} 4$. This small matrix size allows us to easily compute the exact Jacobian matrix every time step. The use of element matrices allows us to increase the mesh size (number of elements) without enlarging the matrix size. The original LCG method treats each element as an independent domain, while the information between sub-domains is explicitly transferred via fluxes. This method was first introduced by [97] for convection-diffusion problems and then generalised to other problems including incompressible Navier-Stokes equations and systemic circulation problems $[12,6,39,98,99,100,101,58]$. The fully explicit version of the LCG method computes multiple solutions at the node shared between two elements. To obtain a unique solution, a posteriori averaging of the multiple solutions was carried out [97]. The proposed SILCG methods also follow an averaging technique that is identical to that of the explicit LCG method. The main difference between the proposed semi-implicit methods and the fully explicit scheme is that all the terms, except for the boundary fluxes, are treated implicitly. The original contributions of this chapter include the introduction of the SILCG method in three versions for systemic blood circulation, demonstration of its simplicity and accuracy against the fully explicit LCG method that has been already developed in $[12,39]$. The chapter is organized into the following sections. In section 2.2 we present the governing equations, numerical procedure, and boundary conditions. In 
section 2.3, a detailed demonstration of the accuracy, robustness and computational efficiency of the proposed methodology is provided. Finally, in section 2.4 , the concluding remarks are reported.

\subsection{Mathematical formulation of the problem}

\subsubsection{Governing equations}

In the current study, the flow is assumed to be incompressible, Newtonian and laminar. The mass and momentum conservation equations for one dimensional flow in compliant vessels may be written, respectively, as $[12,102]$

$$
\begin{gathered}
\frac{\partial A}{\partial t}+\frac{\partial(A u)}{\partial x}=0 \\
\frac{\partial u}{\partial t}+u \frac{\partial u}{\partial x}+\frac{1}{\rho} \frac{\partial p}{\partial x}-\frac{f}{\rho}=0
\end{gathered}
$$

where $A$ is the cross sectional area, $u$ is the average velocity over such section and $\rho$ is the fluid density. The term $f$ represents the friction forces due to viscosity and it is given as [12]

$$
f=\frac{-8 \pi \mu u}{A}
$$

in which $\mu$ is the fluid viscosity. The pressure $p$ is linked to the area via a non-linear relationship, given as [39, 103, 104, 105]

$$
p=p_{\text {ext }}+\beta\left(\sqrt{A}-\sqrt{A_{0}}\right)
$$

where $p_{\text {ext }}$ is the pressure from the surrounding tissues, $A_{0}$ is the area at zero transmural pressure and $\beta$ accounts for the material properties of the elastic vessel and given by

$$
\beta=\frac{\sqrt{\pi} h E}{A_{0}\left(1-\sigma^{2}\right)}
$$


in which $h$ is the vessel wall thickness, $E$ is the Young's modulus and $\sigma$ is the Poisson's ratio, assumed to be 0.5 (i.e. the vessel wall is incompressible). The system (2.1) and (2.2) can be presented in the following compact form [39, 12]

$$
\frac{\partial \mathbf{U}}{\partial t}=\mathbf{S}-\frac{\partial \mathbf{F}}{\partial x}
$$

where

$$
\mathbf{U}=\left[\begin{array}{c}
A \\
u
\end{array}\right], \quad \mathbf{F}=\left[\begin{array}{c}
A u \\
\frac{u^{2}}{2}+\frac{1}{\rho}\left[p_{e x t}+\beta\left(\sqrt{A}-\sqrt{A_{0}}\right)\right]
\end{array}\right] \quad \text { and } \quad \mathbf{S}=\left[\begin{array}{c}
0 \\
-\frac{8 \pi \mu}{\rho} \frac{u}{A}
\end{array}\right]
$$

\subsubsection{Numerical schemes}

Here, the three variants of the SILCG formulation are presented.

\section{SILCG-TE}

The proposed methodology is based on a LCG spatial discretization scheme, stabilised with a Taylor expansion based method [12, 39, 40]. Differentiating Eq. (2.6) with respect to time and applying the chain rule yields

$$
\frac{\partial^{2} \mathbf{U}}{\partial t^{2}}=\mathbf{C} \frac{\partial \mathbf{U}}{\partial t}-\frac{\partial}{\partial x}\left(\mathbf{H} \frac{\partial \mathbf{U}}{\partial t}\right)
$$

where $\mathbf{C}=\partial \mathbf{S} / \partial \mathbf{U}$ and $\mathbf{H}=\partial \mathbf{F} / \partial \mathbf{U}$ are the Jacobian matrices. The Taylor expansion in time of the primitive variables $(A$ and $u$ ), restricted to the second order terms, reads

$$
\mathbf{U}^{n+1}=\mathbf{U}^{n}+\Delta t \frac{\mathbf{U}}{\partial t}^{n+\theta}+\frac{\Delta t^{2}}{2} \frac{\partial^{2} \mathbf{U}^{n+\theta}}{\partial t^{2}}
$$

where $\theta$ is a parameter $(0 \leq \theta \leq 1)$ controlling the time integration scheme. By inserting (2.6) and (2.8) into Eq. (2.9), it is possible to obtain the following semi-discrete form [39]

$$
\frac{\mathbf{U}^{n+1}-\mathbf{U}^{n}}{\Delta t}=\left\{\mathbf{S}-\frac{\partial \mathbf{F}}{\partial x}+\frac{\Delta t}{2}\left[-\mathbf{C}\left(\frac{\partial \mathbf{F}}{\partial x}-\mathbf{S}\right)+\frac{\partial}{\partial x} \mathbf{H}\left(\frac{\partial \mathbf{F}}{\partial x}-\mathbf{S}\right)\right]\right\}^{n+\theta} .
$$


The primitive variables are approximated in space by linear standard finite element shape functions, i.e.

$$
A=\mathbf{N} \tilde{\mathbf{A}}, \quad u=\mathbf{N} \tilde{\mathbf{u}} .
$$

In the above equation

$$
\mathbf{N}=\left[\begin{array}{llll}
N_{1} & N_{2} & \ldots & N_{m}
\end{array}\right]^{T}, \quad \tilde{\mathbf{A}}=\left[\begin{array}{llll}
\tilde{A}_{1} & \tilde{A}_{2} & \ldots & \tilde{A}_{m}
\end{array}\right], \quad \tilde{\mathbf{u}}=\left[\begin{array}{llll}
\tilde{u}_{1} & \tilde{u}_{2} & \ldots & \tilde{u}_{m}
\end{array}\right]
$$

where $\mathbf{N}$ contains $m$ nodal shape functions defined over a spatial domain $\Omega$, whilst $\tilde{\mathbf{A}}$ and $\tilde{\mathbf{u}}$ are the nodal values of the primitive variables [106]. For the sake of simplicity, Eq. (2.11) can be rewritten in the compact form as $\mathbf{U}=\mathbf{N} \tilde{\mathbf{U}}$. The same discretization is carried out for the vectors $\mathbf{F}=\mathbf{N} \tilde{\mathbf{F}}$ and $\mathbf{S}=\mathbf{N} \tilde{\mathbf{S}}$. Applying the Galerkin weighting to Eq. (2.10), expressed over an elemental sub-domain $\Omega_{e}$ with boundary $\Gamma_{e}$, yields

$$
\left[\mathbf{M}_{e}\right] \Delta \tilde{\mathbf{U}}^{n}-\Delta t\left(\left[\mathbf{K}_{e}\right] \tilde{\mathbf{F}}^{n+\theta}+\left[\mathbf{L}_{e}\right] \tilde{\mathbf{S}}^{n+\theta}+\mathbf{f}_{\Gamma_{e}}^{n+\theta}\right)=0
$$

where $\mathbf{M}_{e}, \mathbf{K}_{e}$ and $\mathbf{L}_{e}$ are respectively the element mass, convective and source matrices, whilst $\mathbf{f}_{\Gamma_{e}}$ is the boundary flux term. We notice that the subscript $e$ refers to the element domain. The fully discrete form of the semi-implicit method, with $\theta=0$ for the boundary fluxes and $\theta=1$ elsewhere, becomes

$$
\left[\mathbf{M}_{e}\right] \Delta \tilde{\mathbf{U}}^{n}-\Delta t\left(\left[\mathbf{K}_{e}\right] \tilde{\mathbf{F}}^{n+1}+\left[\mathbf{L}_{e}\right] \tilde{\mathbf{S}}^{n+1}+\mathbf{f}_{\Gamma_{e}}^{n}\right)=0
$$

As seen in the above equation, the interface LCG fluxes are treated at time level $n$ and thus the method is semi-implicit. Making these fluxes implicit may require the solution of additional equations, and the introduced approximations may lead to inaccuracies. The mass matrix in the above equation is defined as

$$
\mathbf{M}_{e}=\frac{l_{e}}{6}\left[\begin{array}{cccc}
2 & 1 & 0 & 0 \\
1 & 2 & 0 & 0 \\
0 & 0 & 1 & 2 \\
0 & 0 & 2 & 1
\end{array}\right]
$$


and it can be lumped for simplifying the solution procedure [107]

$$
\mathbf{M}_{e}=\frac{l_{e}}{2}\left[\begin{array}{llll}
1 & 0 & 0 & 0 \\
0 & 1 & 0 & 0 \\
0 & 0 & 1 & 0 \\
0 & 0 & 0 & 1
\end{array}\right]
$$

where $l_{e}$ is the element length. In the current study, lumped mass matrices are used in all the calculations. The other matrices introduced are defined as

$$
\begin{aligned}
& \mathbf{K}_{e}=\frac{1}{2}\left[\begin{array}{cccc}
-1 & -1 & 0 & 0 \\
1 & 1 & 0 & 0 \\
0 & 0 & -1 & -1 \\
0 & 0 & 1 & 1
\end{array}\right]+\frac{\Delta t}{4} \mathbf{C}\left[\begin{array}{cccc}
-1 & -1 & 0 & 0 \\
1 & 1 & 0 & 0 \\
0 & 0 & -1 & -1 \\
0 & 0 & 1 & 1
\end{array}\right] \\
& -\frac{\Delta t}{2 l_{e}} \mathbf{H}\left[\begin{array}{cccc}
1 & -1 & 0 & 0 \\
-1 & 1 & 0 & 0 \\
0 & 0 & 1 & -1 \\
0 & 0 & -1 & 1
\end{array}\right] \\
& \mathbf{L}_{e}=\frac{l_{e}}{6}\left[\begin{array}{cccc}
2 & 1 & 0 & 0 \\
1 & 2 & 0 & 0 \\
0 & 0 & 2 & 1 \\
0 & 0 & 1 & 2
\end{array}\right]+\frac{\Delta t l_{e}}{12} \mathbf{C}\left[\begin{array}{cccc}
2 & 1 & 0 & 0 \\
1 & 2 & 0 & 0 \\
0 & 0 & 2 & 1 \\
0 & 0 & 1 & 2
\end{array}\right] \\
& +\frac{\Delta t}{4} \mathbf{H}\left[\begin{array}{cccc}
-1 & -1 & 0 & 0 \\
1 & 1 & 0 & 0 \\
0 & 0 & -1 & -1 \\
0 & 0 & 1 & 1
\end{array}\right] \text {. }
\end{aligned}
$$


The vectors of Eq. (2.14) are expressed as

$$
\begin{array}{r}
\Delta \tilde{\mathbf{U}}^{n}=\left\{\begin{array}{c}
\Delta \tilde{A_{1}} \\
\Delta \tilde{A_{2}} \\
\Delta \tilde{u_{1}} \\
\Delta \tilde{u_{2}}
\end{array}\right\}^{n}, \tilde{\mathbf{F}}^{n+1}=\left\{\begin{array}{c}
(\tilde{A} \tilde{u})_{1} \\
(\tilde{A} \tilde{u})_{2} \\
\tilde{u}_{1}^{2}+\frac{1}{\rho}\left[p_{\text {ext }}+\beta\left(\sqrt{\tilde{A}}-\sqrt{A_{0}}\right)\right]_{1} \\
\frac{\tilde{u}_{2}^{2}}{2}+\frac{1}{\rho}\left[p_{\text {ext }}+\beta\left(\sqrt{\tilde{A}}-\sqrt{A_{0}}\right)\right]_{2}^{n+1}
\end{array}\right. \\
\tilde{\mathbf{S}}^{n+1}=\left\{\begin{array}{c}
0 \\
0 \\
-\frac{8 \pi \mu}{\rho} \tilde{u}_{1} \\
-\frac{8 \pi \mu}{\rho} \tilde{A}_{1} \\
\tilde{A}_{2}
\end{array}\right\}
\end{array}
$$

The subscripts 1 and 2 refer respectively to first and second nodes of the element (see Appendix A for more details about the finite element procedure). The Jacobian matrices can be written as

$$
\begin{aligned}
& \mathbf{H}=\left[\begin{array}{cccc}
u_{\text {ave }} & 0 & A_{\text {ave }} & 0 \\
0 & u_{\text {ave }} & 0 & A_{\text {ave }} \\
\frac{\beta_{1}}{2 \rho \sqrt{A_{\text {ave }}}} & 0 & u_{\text {ave }} & 0 \\
0 & \frac{\beta_{2}}{2 \rho \sqrt{A_{\text {ave }}}} & 0 & u_{\text {ave }}
\end{array}\right] \\
& \mathbf{C}=\left[\begin{array}{cccc}
0 & 0 & 0 & 0 \\
0 & 0 & 0 & 0 \\
\frac{8 \pi \mu}{\rho} \frac{u_{\text {ave }}^{2}}{A_{\text {ave }}^{2}} & 0 & -\frac{8 \pi \mu}{\rho} \frac{1}{A_{\text {ave }}} & 0 \\
0 & \frac{8 \pi \mu}{\rho} \frac{u_{\text {ave }}}{A_{\text {ave }}^{2}} & 0 & -\frac{8 \pi \mu}{\rho} \frac{1}{A_{\text {ave }}}
\end{array}\right] .
\end{aligned}
$$

Note that the entries of the Jacobian matrices ( $\mathbf{H}$ and $\mathbf{C}$ ) are averaged over the element. For example, for calculating $A_{1}$, the first line of the matrix $\mathbf{H}$ is used as $u_{\text {ave }}=\left(\tilde{u}_{1}+\tilde{u}_{2}\right) / 2$ and $A_{\text {ave }}=\left(\tilde{A}_{1}+\tilde{A}_{2}\right) / 2$. Similar procedure is applied for the other quantities in the matrix H. The same approach is used for computing matrix $\mathbf{C}$. We notice that, in CG method, the flux term in Eq. (2.14) is equal to 0, except at the boundaries. In the LCG method however, we use this term to link elements and thus the normal assembly procedure is 
A

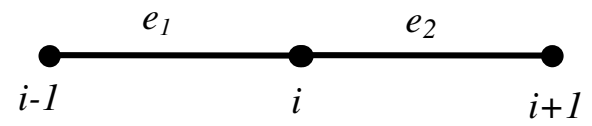

B
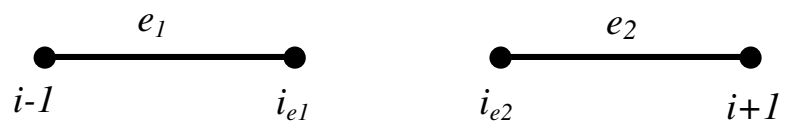

Figure 2.1: One-dimensional elements in CG (A) and LCG methods (B).

avoided here. This is why the LCG method is explicitly, locally conservative rather than implicitly conservative as in the case of CG methods [101]. The definition of fluxes $\tilde{\mathbf{F}}$ is given in Eq. (2.19). We use appropriate outward pointing normals to define the fluxes at both ends of an element, i.e. $\mathbf{f}_{\Gamma_{e}}^{n}=\tilde{\mathbf{F}}^{n}$. These fluxes must be recalled from the previous time step (i.e. $n$ time level) for maintaining continuity (see $[97,98]$ for more details). An alternative would be introducing very expensive additional implicit approximations for the fluxes to maintain explicit local conservation. Thus, the methods proposed at the best can only be semi-implicit. Figure 2.1 shows, respectively, the CG and LCG element discretizations. While CG method uses a continuous discretization between elements, the LCG method breaks the link, which is then reestablished through edge fluxes a posteriori. Eq. (2.14) is solved independently over the elements $e_{1}$ and $e_{2}$ and a unique nodal solution is obtained by averaging the variables on the intermediate nodes as $[97,98]$

$$
\tilde{\mathbf{U}}_{i}=\frac{\tilde{\mathbf{U}}_{i e 1}+\tilde{\mathbf{U}}_{i e 2}}{2}
$$

where subscripts $i e 1$ and $i e 2$ refer to the two solutions obtained at node $i$.

\section{SILCG-SUPG}

With the streamline upwind Petrov-Galerkin (SUPG) method, the test function is modified in order to stabilise the oscillations due to the convective term

$$
\mathbf{W}=\mathbf{N}+\frac{\alpha l_{e}}{2} \frac{\tilde{u}_{i}}{\left|u_{\text {ave }}\right|} \frac{d \mathbf{N}}{d x},
$$


where $\mathbf{W}$ is a modified shape function of the linear weighting function $\mathbf{N}$. The value of $\alpha$ varies depending on local Peclet number $P e$ and it can be optimally calculated as [108]

$$
\alpha=\operatorname{coth}(P e)-\frac{1}{P e} \text { and } P e=\frac{\left|u_{a v e}\right| l_{e}}{2 v},
$$

in which $v$ is the kinematic viscosity of the fluid. The rest of the formulation is identical to that of the SILCG-TE method presented in the previous subsection.

\section{SILCG-BTCS}

The last method we introduce is the backward in time and central (standard Galerkin method) in space (BTCS) method in its semi-implicit form. The motivation for this comes from the fact that the implicit form of such method for linear convection equations is unconditionally stable [109]. The idea here is therefore to investigate the limits of such a method in the context of the semi-implicit discretization. The method is identical to either SILCG-TE or SILCG-SUPG method without the additional stabilization terms. In other words, the matrices are reduced by taking both matrices $\mathbf{H}$ and $\mathbf{C}$ equal to 0 .

\subsubsection{Solution procedure}

The solution of the system defined in Eq. (2.14) is sought by employing the Newton's method $[108,110]$. The iterative solution of the unknown variables vector $\tilde{\mathbf{U}}$ (i.e. $\tilde{\mathbf{U}}=$ $\left[\begin{array}{llll}\tilde{A}_{1} & \tilde{A}_{2} & \tilde{u}_{1} & \tilde{u}_{2}\end{array}\right]^{T}$ ) at the iteration $k+1$ is given by

$$
\tilde{\mathbf{U}}^{k+1}=\tilde{\mathbf{U}}^{k}+\delta \tilde{\mathbf{U}}^{k}
$$

The update $\delta \tilde{\mathbf{U}}^{k}$ is estimated via

$$
\mathbf{J}\left(\tilde{\mathbf{U}}^{k}\right) \delta \tilde{\mathbf{U}}^{k}=-\mathbf{R}\left(\tilde{\mathbf{U}}^{k}\right)
$$

where $\mathbf{J}\left(\tilde{\mathbf{U}}^{k}\right)$ is a 4 x 4 Jacobian matrix and $\mathbf{R}\left(\tilde{\mathbf{U}}^{k}\right)$ is the residual vector of Eq. (2.14). Eq. (2.26) is iteratively solved until convergence is reached. If the initial guess is far 
from the root, Newton method may not converge. In the current work, the variables are initialised at each iteration with the values of the previous time step [111]. A linear representation of Eq. (2.26) gives four linear algebraic equations per element (i.e. $R_{A_{1}}, R_{A_{2}}, R_{u_{1}}$ and $R_{u_{2}}$ ). The detailed form may now be written as

$$
\left[\begin{array}{llll}
\frac{\partial R_{A_{1}}}{\partial A_{1}} & \frac{\partial R_{A_{1}}}{\partial A_{2}} & \frac{\partial R_{A_{1}}}{\partial u_{1}} & \frac{\partial R_{A_{1}}}{\partial u_{2}} \\
\frac{\partial R_{A_{2}}}{\partial A_{1}} & \frac{\partial R_{A_{2}}}{\partial A_{2}} & \frac{\partial R_{A_{2}}}{\partial u_{1}} & \frac{\partial R_{A_{2}}}{\partial u_{2}} \\
\frac{\partial R_{u_{1}}}{\partial A_{1}} & \frac{\partial R_{u_{1}}}{\partial A_{2}} & \frac{\partial R_{u_{1}}}{\partial u_{1}} & \frac{\partial R_{u_{1}}}{\partial u_{2}} \\
\frac{\partial R_{u_{2}}}{\partial A_{1}} & \frac{\partial R_{u_{2}}}{\partial A_{2}} & \frac{\partial R_{u_{2}}}{\partial u_{1}} & \frac{\partial R_{u_{2}}}{\partial u_{2}}
\end{array}\right]\left\{\begin{array}{c}
\delta \tilde{A}_{1} \\
\delta \tilde{A}_{2} \\
\delta \tilde{u}_{1} \\
\delta \tilde{u}_{2}
\end{array}\right\}=-\left\{\begin{array}{c}
R_{A_{1}} \\
R_{A_{2}} \\
R_{u_{1}} \\
R_{u_{2}}
\end{array}\right\} .
$$

The linear system presented above is solved by using LU factorisation [112]. The global system solution is computed at each time step via the following algorithm

\section{Algorithm 1}

1. do $\mathrm{i}=1, \mathrm{~m} \quad \mathrm{~m}=1,2, \ldots . .$, elements

2. do $\mathrm{j}=1,2 \quad$ nodes

3. initialise $\tilde{\mathbf{U}}^{k, n+1}=\tilde{\mathbf{U}}^{k, n}$

4. end do

5. do $\mathrm{k}=1$, number of iterations

6. compute $\mathbf{J}\left(\tilde{\mathbf{U}}^{k}\right)$ and $\mathbf{R}\left(\tilde{\mathbf{U}}^{k}\right)$

7. call LU and solve for $\delta \tilde{\mathbf{U}}^{k}$ through Eq. (2.26)

8. evaluate, if $\delta \tilde{\mathbf{U}}^{k} \leq$ tolerance

yes $\rightarrow$ go step 10

no $\rightarrow$ put $\tilde{\mathbf{U}}^{k+1}=\tilde{\mathbf{U}}^{k}$

9. end do

10. update through Eq. (2.25)

11. end do 
Steps 2-4 represent the element initialisation procedure (where SILCG holds both elements and nodes in different loops whereas CG has only nodes). Steps from 5 to 10 refer to the Newton iteration. The stopping tolerance for the algorithms is set equal to $10^{-4}$.

\subsubsection{Network boundary conditions}

For prescribing boundary conditions, the methodology proposed by in [39, 12] is followed. With regard to the inlet flow condition, two types of boundaries can generally be used. The reflective boundaries, where values of $A, u$, and $p$ (or commonly used flow rate $Q=A u)$ are directly prescribed at the inlet node. Usually, any of these variables can be expressed as a function of time (see for instance [40, 44]). Alternatively, the non-reflective boundaries where characteristic variables are used. The latter methods are more realistic and here the boundary conditions are calculated as a combination of outside and inside conditions by implementing Riemann method [39]. Since the wave nature of the problem is exploited by the latter method, we use this approach for specifying the boundary conditions. As a part of the wave comes from the domain interior, the boundaries need to be extrapolated from the previous time step. The problem is initialized using a specified wave of $p_{\text {init }}=50 \mathrm{mmHg}$ [12]. The prescribed wave from outside the domain is the pressure waveform as shown in Figure 2.2. In other words, the pressure is defined at any time step at the inlet including initial pressure. This pressure will be used later to calculate the area at the inlet. By assuming $\mathbf{S} \approx 0$ near the boundaries and applying the chain rule to the spatial derivative, Eq. (2.6) becomes

$$
\frac{\partial \mathbf{U}}{\partial t}+\mathbf{H} \frac{\partial \mathbf{U}}{\partial x}=0
$$

The characteristic speeds for the system are the eigenvalues of the matrix $\mathbf{H}$, which can be obtained by solving $|\mathbf{H}-\lambda \mathbf{I}|=0$, with $\mathbf{I}$ being the identity matrix. The characteristic speeds are given as

$$
\lambda_{f, b}=u \pm c
$$




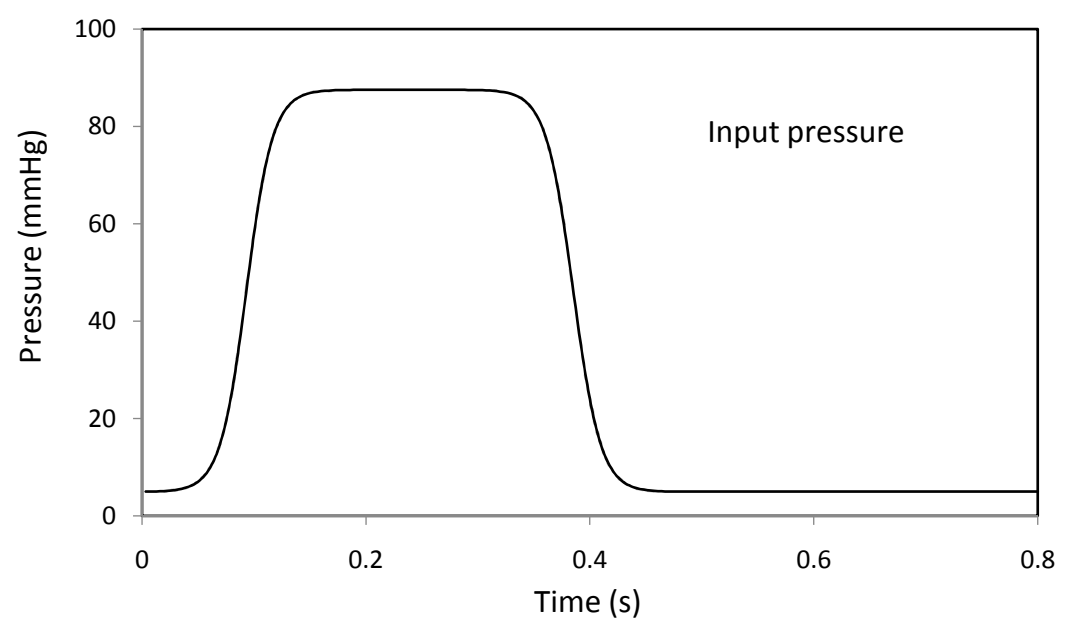

Figure 2.2: Input pressure wave.

where the subscripts $f$ and $b$ refer to forward and backward travelling directions, and $c$ is the pulse wave speed which is given as

$$
c=\sqrt{\frac{\beta}{2 \rho}} A^{1 / 4} .
$$

The wave speed $c$ is much greater than fluid velocity $u$ under physiological flow conditions, and therefore $\lambda_{f}=u+c>0$ while $\lambda_{b}=u-c<0$. These two eigenvalues are real, so the system is strictly hyperbolic. This needs only one boundary condition at each inlet and exit of the domain [39]. The matrix $\mathbf{L}$ of the left eigenvalues of $\mathbf{H}$ can be written as

$$
\mathbf{L}=\frac{\partial \mathbf{V}}{\partial \mathbf{U}}=\left[\begin{array}{cc}
\frac{c}{A} & 1 \\
-\frac{c}{A} & 1
\end{array}\right],
$$

where $\mathbf{V}=\left[\begin{array}{ll}w_{f} & w_{b}\end{array}\right]^{T}$, and then

$$
\begin{gathered}
\mathbf{L H}=\Lambda \mathbf{L}, \\
\Lambda=\left[\begin{array}{cc}
\lambda_{f} & 0 \\
0 & \lambda_{b}
\end{array}\right] .
\end{gathered}
$$

Multiplying Eq. (2.28) by the matrix $\mathbf{L}$ and substituting $\mathbf{L H}$ from Eq. (2.32) gives

$$
\mathbf{L} \frac{\partial \mathbf{U}}{\partial t}+\Lambda \mathbf{L} \frac{\partial \mathbf{U}}{\partial x}=0
$$


Note that $\mathbf{H}=\mathbf{L}^{-1} \Lambda \mathbf{L}$, and thus the above equation can be rewritten as

$$
\frac{\partial \mathbf{V}}{\partial t}+\Lambda \frac{\partial \mathbf{V}}{\partial x}=0
$$

The characteristic variables can now be determined as

$$
w_{f, b}=u \pm 4 c .
$$

The primitive variables may be estimated from the characteristic variables as $[39,113]$

$$
\begin{gathered}
A=\frac{\left(w_{f}-w_{b}\right)^{4}}{1024}\left(\frac{\rho}{\beta}\right)^{2}, \\
u=\frac{w_{f}+w_{b}}{2} .
\end{gathered}
$$

The equations above show that a pair of the characteristic variables is necessary for specifying one boundary condition. At the inlet, $w_{f}$ implies the imposed pressure shown in Figure 2.2; thus rearranging Eq. (2.37) gives [39, 12]

$$
w_{f}^{n+1}=w_{b}^{0}+8 A^{1 / 4} \sqrt{\frac{\beta}{2 \rho}},
$$

where $w_{b}^{0}$ is the initial backward variable, and it is equal to $w_{b}$ at any time (i.e. assume no backward waves reach the inlet [39]. This is possible at the inlet as $p_{\text {int }}$ is given (see Figure 2.2). Similarly, at first time step where $p_{\text {int }}$ is known and thus initial $A$ can be computed by rearranging Eq. (2.4). Thus Eq. (2.39) is now rewritten in terms of the pressure using Eq. (2.4) as

$$
w_{f}^{n+1}=w_{b}^{0}+4 \sqrt{\frac{2}{\rho}} \sqrt{p-p_{\text {ext }}+\beta \sqrt{A_{0}}} .
$$

As mentioned before, the pressure $p$ is known at the inlet. Backward variable $w_{b}$ carries information from domain interior, so it is extrapolated according to the following equation $[39,12]$

$$
\left.w_{b}^{n+1}\right|_{x=x_{0}}=w_{\left.b\right|_{x=x_{0}} ^{n}-\lambda_{b}^{n} \Delta t} .
$$


At this stage both variables $w_{f}$ and $w_{b}$ at the inlet are calculated as shown in Figure 2.3 and both boundaries (i.e. $A$ and $u$ at the inlet) can be now estimated through Eqs. (2.37) and (2.38). This figure represents a general case for an artery with inlet and outlet boundaries. For prescribing the velocity, similar procedure may be applied to Eq. (2.38), so that

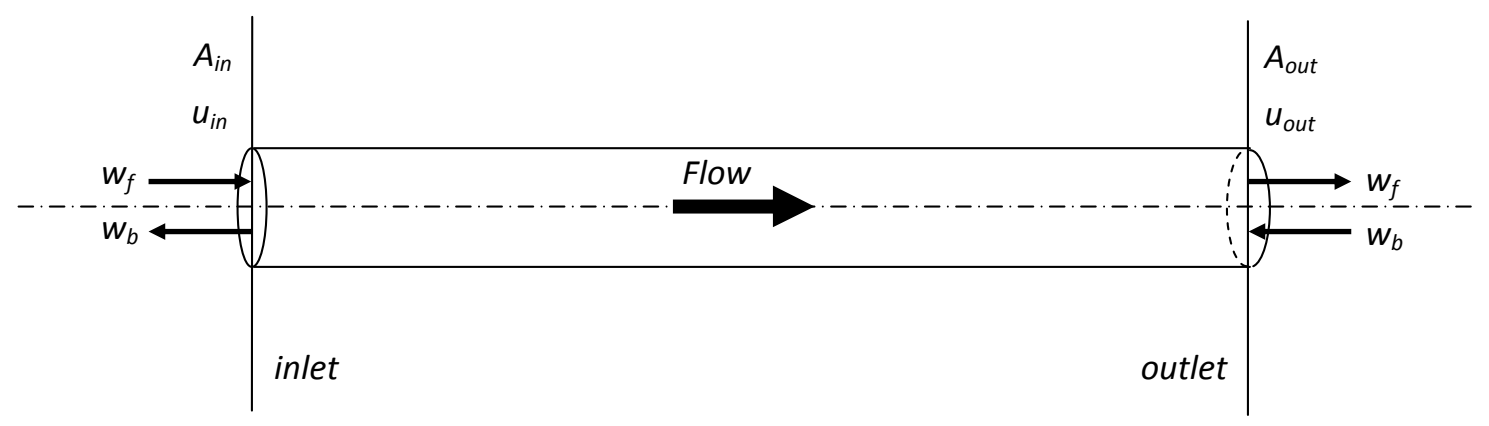

Figure 2.3: A typical arterial segment with inlet and outlet boundaries.

$$
w_{f}^{n+1}=2 u-w_{b}^{0} .
$$

The velocity at the beginning is set equal to 0 for the first time step and then the velocity from previous time step can be used for the new time level. This is another way to obtain either the inlet velocity through Eq. (2.38) or the forward characteristic variable $w_{f}$ when the velocity is given.

With regard to the terminal boundaries, the same Eqs. (2.37) and (2.38) can be used to estimate the area and velocity shown in Figure 2.3 at the outlet but we need to obtain new characteristics variables. So, $w_{b}$ was an outgoing variable at the inlet, now it is an incoming one. It represents the impedance, and it is calculated by using the tapering vessel approach. In this approach, the area $A$ of an arterial segment decreases gradually along the length (i.e. the outlet area is reduced by $10 \%$ from the area at the inlet [12]). Thus, additional 28 segments have been added at each terminal artery to create this impedance and captured by the the characteristic variable as boundary condition. Pure reflections are 
assumed, and thus $w_{b}$ can be determined as

$$
w_{b}^{n+1}=w_{b}^{0}
$$

Meanwhile, $w_{f}$ is extrapolated by adopting $[12,39]$

$$
\left.w_{f}^{n+1}\right|_{x=x_{L}}=w_{\left.f\right|_{x=x_{L}}-\lambda_{f}^{n} \Delta t} .
$$

It is obvious that $w_{f}$ is an outgoing variable at the outlet and carries information from the inside domain as shown in Figure 2.3.

Moreover, the characteristic system is useful to enforce quantities at bifurcations and other discontinuities. Here, we consider two cases as shown in Figure 2.4. Figure 2.4A shows a case of single tube with a sudden change in the $A$ or $\beta$, representing a discontinuity. Figure 2.4B shows the case of a parent vessel branches and its two daughter branches. For each branching location, there are six unknowns, which are $A$ and $u$ for parent (p)

A

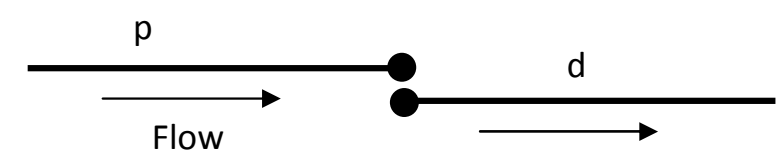

B

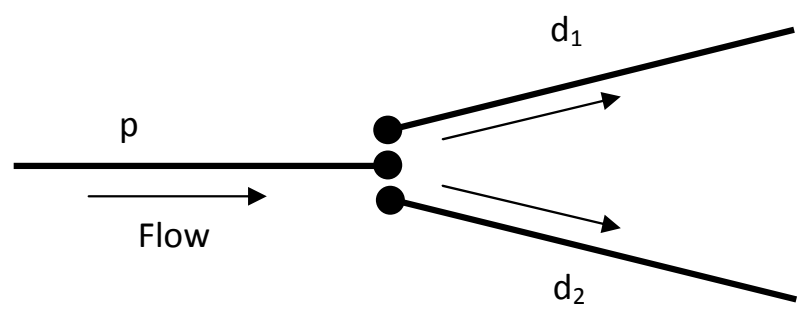

Figure 2.4: Different types of arterial tree discontinuities.

and daughter (d) segments. For the previous case (Figure 2.4A), there will be four unknowns. Such variables are computed by assembling a system of equations accounting for mass and momentum conservation, and definitions of characteristic speeds [39]. The 
conservation of mass can be simply written as

$$
Q_{p}=\sum_{i=1}^{N} Q_{i},
$$

where $\mathrm{N}$ is number of daughter vessels. In Eq. (2.45) the flow direction is assumed to be from parent to daughter vessels. The continuity of total pressure (Bernoulli's equation) between the parent and daughter vessels may be expressed as

$$
\frac{\rho u_{p}^{2}}{2}+p_{s_{p}}=\frac{\rho u_{i}^{2}}{2}+p_{s_{i}}
$$

where $\rho u^{2} / 2$ is the dynamic pressure and $p_{s}$ represents the static pressure given in Eq. (2.4). The characteristic equations for the parent and daughter vessels are

$$
\begin{aligned}
& w_{f_{p}}=u_{p}+4 A_{p}^{1 / 4} \sqrt{\frac{\beta_{p}}{2 \rho}}, \\
& w_{b_{i}}=u_{i}-4 A_{i}^{1 / 4} \sqrt{\frac{\beta_{i}}{2 \rho}} .
\end{aligned}
$$

Figure $2.4 \mathrm{~A}$ is similarly represented with four unknowns.

\subsection{Results and discussions}

In this section, the accuracy and computational efficiency of the proposed semi-implicit methods are discussed. The well established explicit Taylor-LCG method [12, 39] is used as a benchmark method to compare the results. For computing the solution, the maximum admissible time step size is adopted. The spatial step used is $l_{e}=0.25 \mathrm{~cm}$ (corresponding to 6288 elements) for all cases unless otherwise stated. The arterial network used in the current study is shown in Figure 2.5. The model consists of 91 arterial segments 63 segments represent the configuration in Figure 2.5 and another 28 segments are used as taper vessels for creating the terminal boundaries. The physical properties of the arterial system considered here is taken from the two publications above along with the original explicit LCG. 


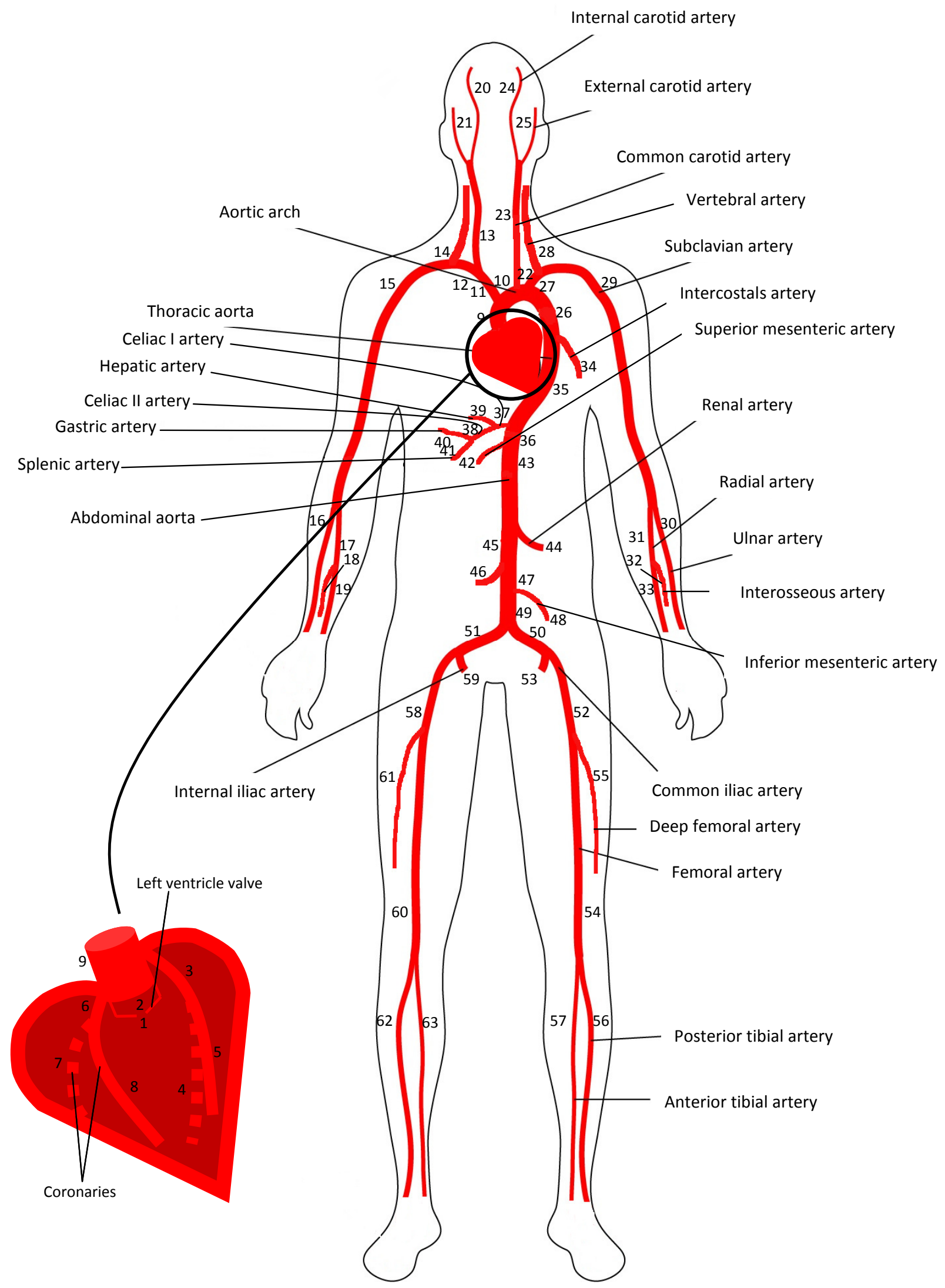

Figure 2.5: Systemic circulation model. 
The duration of a cardiac cycle is assumed to be $\approx 0.8 s$ or $70-75$ beats $/ \mathrm{min}$ and the opening ventricular valve duration is $0.057 s$ while the closing duration is $0.039 s$. A coronary model as in [39] is also included. The blood viscosity and density are set equal to, respectively, $0.035 \mathrm{~g} /(\mathrm{cm} \mathrm{s})$ and $1.06 \mathrm{~g} / \mathrm{cm}^{3}$. More details on the morphology and structural properties of the arterial system can be found in $[12,39]$.

\subsubsection{Wave-forms along the network}

Figures 2.6 and 2.7 show, for all the SILCG methods, the pressure and flow time evolution at different locations along the arterial tree. The monitored segments are labelled 9, 13, $23,15,29,60,54,35$ and 49 according to Figure 2.5. Results obtained by using the explicit LCG are also included. For the semi-implicit methods, the Jacobian matrix is updated at each iteration. The solutions computed with new schemes agree very well with each other and with the one obtained with the explicit method. The calculations show that the SILCG-BTCS admits a maximum time step equal to $\Delta t=0.1 \mathrm{~ms}$, while for the SILCG-TE and SILCG-SUPG methods larger time step sizes can be selected, i.e. $\Delta t=0.15 \mathrm{~ms}$ and $\Delta t=0.145 \mathrm{~ms}$ respectively. As expected, the additional stabilization improves the stability and allows for larger time step values. Table 2.1 shows all time steps used and corresponding CFL number for each method. The CFL number can be defined as [40]

$$
\mathrm{CFL}=\frac{\lambda \Delta t}{l_{e}},
$$

where $\lambda$ is the maximum absolute velocity given in the characteristic speed in Eq. (2.29), $\Delta t$ and $l_{e}$ are the time and spatial steps respectively.

At this stage, we estimate the root mean square relative error (RMSE) for pressure and flow as shown in Table 2.2. These errors are defined as [40]

$$
R M S E_{p}=\sqrt{\frac{1}{n} \sum_{i=1}^{n}\left(\frac{p_{S I L C G}^{i}-p_{L C G}^{i}}{p_{L C G}^{i}}\right)^{2}}
$$




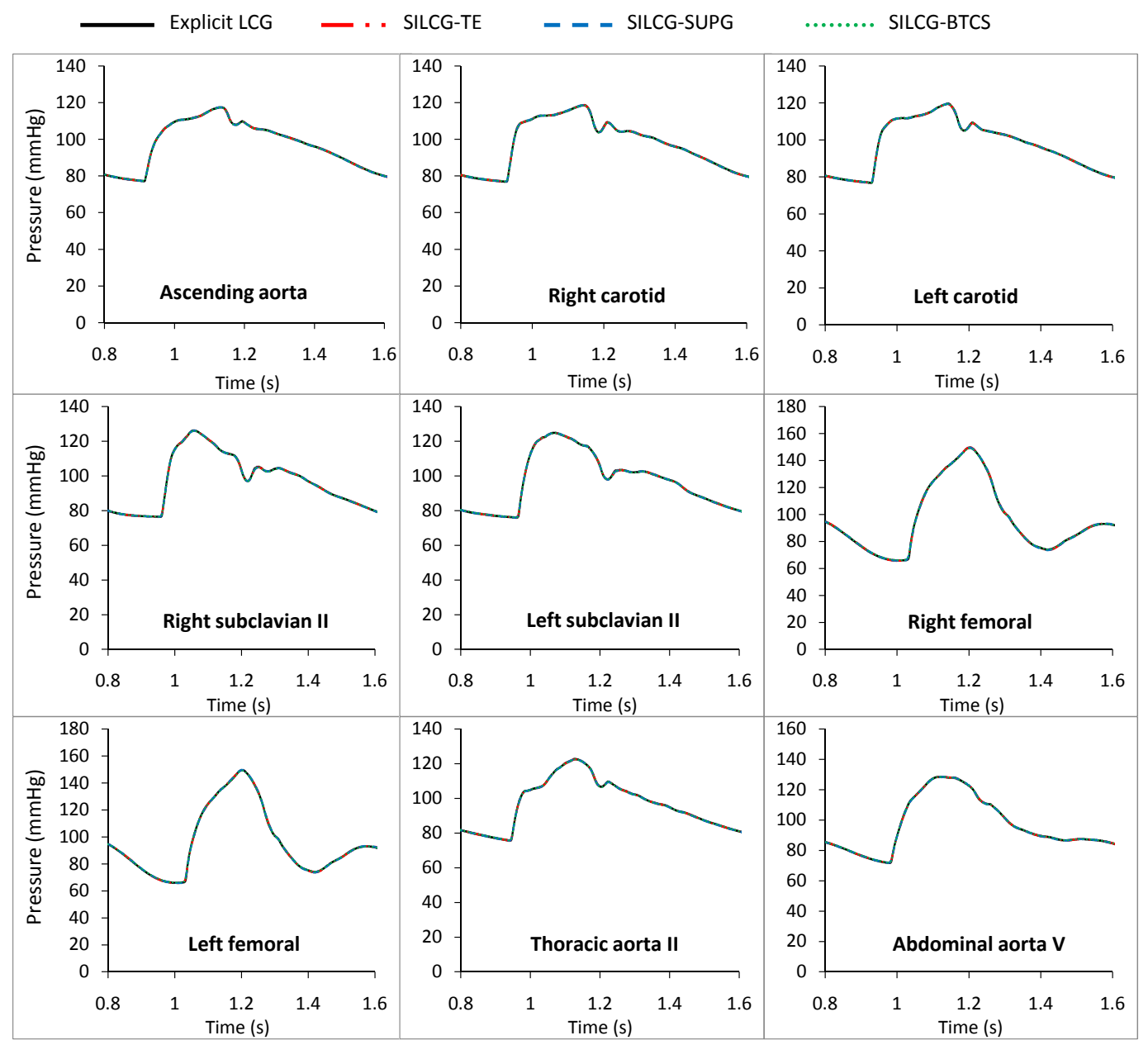

Figure 2.6: Pressure variation in different arterial segments.

Table 2.1: Time steps considered and related CFL, $l_{e}=0.25 \mathrm{~cm}$.

\begin{tabular}{cccc}
\hline Parameter & SILCG-TE & SILCG-SUPG & SILCG-BTCS \\
\hline$\Delta t_{\max }(m s)$ & 0.15 & 0.145 & 0.1 \\
$\mathrm{CFL}$ & 1.104 & 1.067 & 0.736 \\
\hline
\end{tabular}




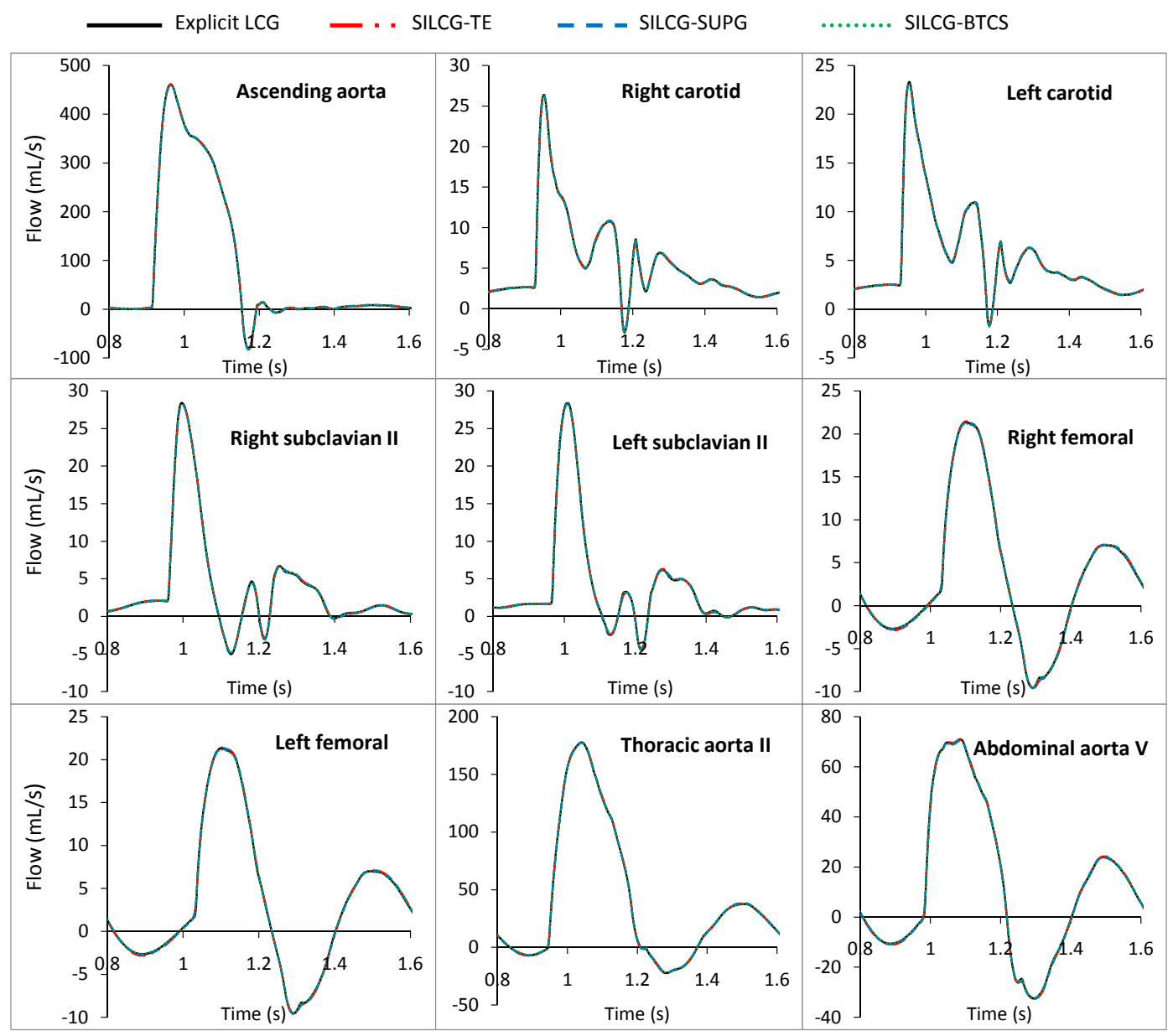

Figure 2.7: Flow variation in various segments.

$$
R M S E_{F}=\sqrt{\frac{1}{n} \sum_{i=1}^{n}\left(\frac{F_{S I L C G}^{i}-F_{L C G}^{i}}{F_{L C G_{\max }}^{i}}\right)^{2}},
$$

where the subscripts $p$ and $F$ refer to pressure and flow respectively. $p_{S I L C G}^{i}$ and $F_{S I L C G}^{i}$ are the estimated values from all proposed approaches at a specific location and for different times $(i=1, \ldots \ldots, n) . \quad p_{L C G}^{i}$ and $F_{L C G}^{i}$ are the reference values considered from [12, 39] (at the same location and time). The table shows that the maximum $R M S E_{p}$ is always below $0.4 \%$, whilst $R M S E_{F}$ is less than $0.7 \%$. Both percentage errors are widely within the range of accuracy expected. Among all methods SILCG-BTCS seems to be the most 
accurate one as shown in Table 2.3. Here, the error percentages in the previous table have been averaged for each method and for all segments. The accuracy comes as a result of removing the stabilisation terms that are associated with the other methods and leading to more accurate approximation.

In Figures 2.8 and 2.9 we show the average number of iterations for reaching convergence against the number of elements employed. In Figure 2.8 the Jacobian matrix is calculated at each iteration, whilst in Figure 2.9 the Jacobian matrix is only calculated once each time step. As seen, the iterations to number of elements ratio is substantially lower than unity. Similar observations were found in [43]. Among the methods presented, the SILCG-BTCS is the fastest as no stabilisation terms are used in this method.

\subsubsection{Spatial convergence}

The effect of mesh refinement on the accuracy is highlighted for various meshes in Figure 2.10. The results are plotted for mid-point of the ascending aorta (i.e. segment 9). A total of three different meshes with element sizes $0.25,0.33$ and $0.5 \mathrm{~cm}$ respectively are tested on SILCG-TE. All other methods give similar results and thus not plotted. The results are compared against a fine mesh solution obtained by using the explicit LCG method. The plot shows that the solution, as the mesh is refined, converges to the results obtained with the explicit LCG method. Also, a case of a very fine mesh is tackled to investigate the accuracy in depth as shown in Figure 2.11. The results are simulated for both blood pressure and flow in the middle of the ascending aorta. The spatial step for the explicit LCG is $l_{e}=0.05 \mathrm{~cm}$ (elements number $=31360$ ) and the proposed approaches are kept as same as before at $l_{e}=0.25 \mathrm{~cm}$ (elements number=6288). This clearly shows the solution is mesh independent with no obvious deviation. This also demonstrates that the mesh size of $l_{e}=0.25 \mathrm{~cm}$ is a good choice. 
Table 2.2: Percentage $R M S E_{p}$ and $R M S E_{F}$ for all cases shown in Figures 2.6 and 2.7.

\begin{tabular}{|c|c|c|c|c|}
\hline Location & Error \% & SILCG-TE & SILCG-SUPG & SILCG-BTCS \\
\hline \multirow[t]{2}{*}{ segment: 9} & $R M S E_{p}$ & 0.038 & 0.052 & 0.087 \\
\hline & $R M S E_{F}$ & 0.151 & 0.154 & 0.199 \\
\hline \multirow[t]{2}{*}{ segment: 13} & $R M S E_{p}$ & 0.069 & 0.078 & 0.098 \\
\hline & $R M S E_{F}$ & 0.371 & 0.380 & 0.293 \\
\hline \multirow[t]{2}{*}{ segment: 23} & $R M S E_{p}$ & 0.070 & 0.078 & 0.100 \\
\hline & $R M S E_{F}$ & 0.386 & 0.399 & 0.321 \\
\hline \multirow[t]{2}{*}{ segment: 15} & $R M S E_{p}$ & 0.133 & 0.136 & 0.122 \\
\hline & $R M S E_{F}$ & 0.406 & 0.411 & 0.307 \\
\hline \multirow[t]{2}{*}{ segment: 29} & $R M S E_{p}$ & 0.146 & 0.151 & 0.133 \\
\hline & $R M S E_{F}$ & 0.437 & 0.441 & 0.316 \\
\hline \multirow[t]{2}{*}{ segment: 60} & $R M S E_{p}$ & 0.360 & 0.344 & 0.280 \\
\hline & $R M S E_{F}$ & 0.651 & 0.617 & 0.434 \\
\hline \multirow[t]{2}{*}{ segment: 54} & $R M S E_{p}$ & 0.359 & 0.342 & 0.280 \\
\hline & $R M S E_{F}$ & 0.650 & 0.616 & 0.434 \\
\hline \multirow[t]{2}{*}{ segment: 35} & $R M S E_{p}$ & 0.090 & 0.097 & 0.109 \\
\hline & $R M S E_{F}$ & 0.250 & 0.240 & 0.219 \\
\hline \multirow[t]{2}{*}{ segment: 49} & $R M S E_{p}$ & 0.152 & 0.157 & 0.145 \\
\hline & $R M S E_{F}$ & 0.580 & 0.529 & 0.401 \\
\hline
\end{tabular}



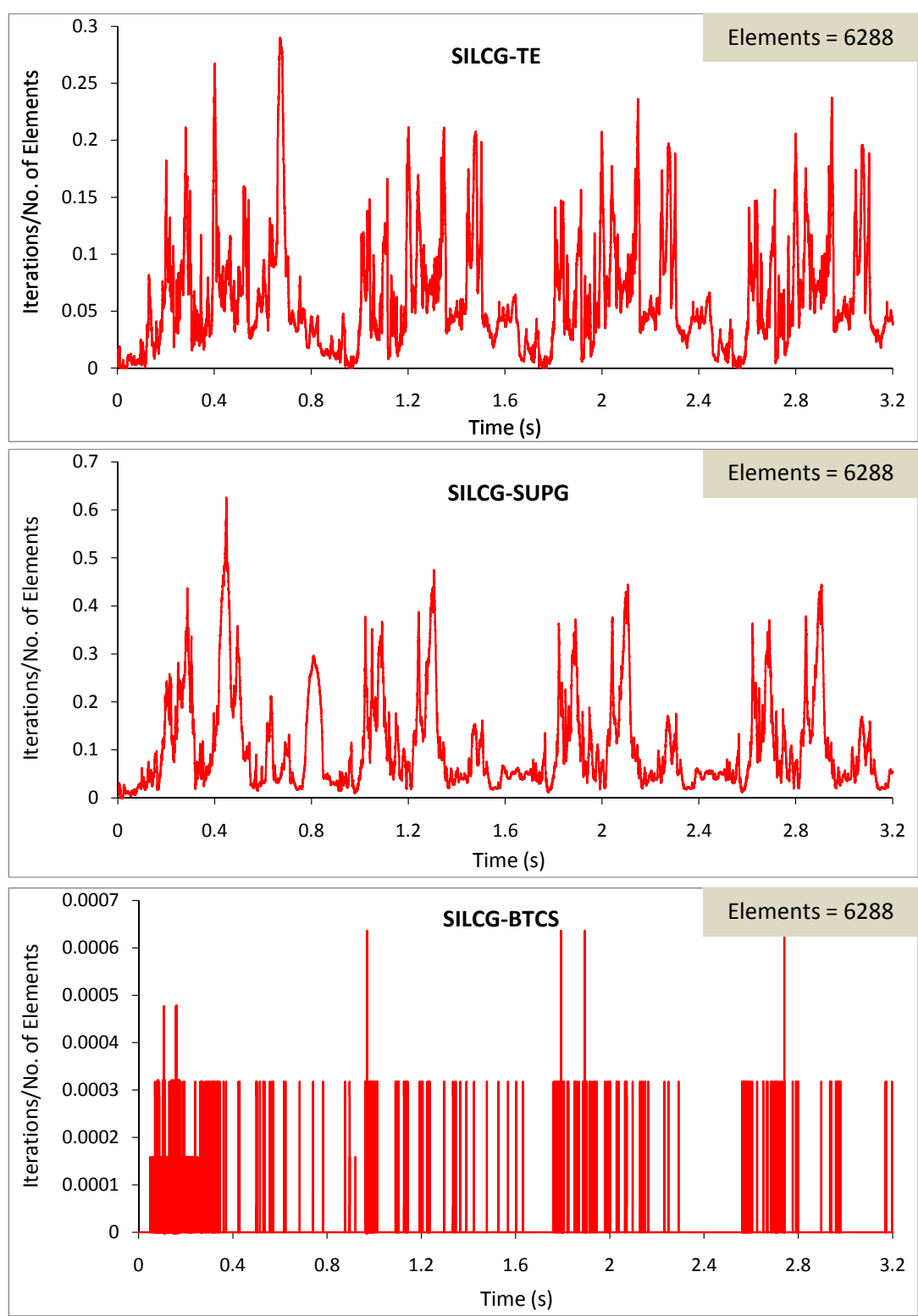

Figure 2.8: Number of iterations to number of elements ratio for for all three approaches. Jacobian matrix is calculated at every iteration. 


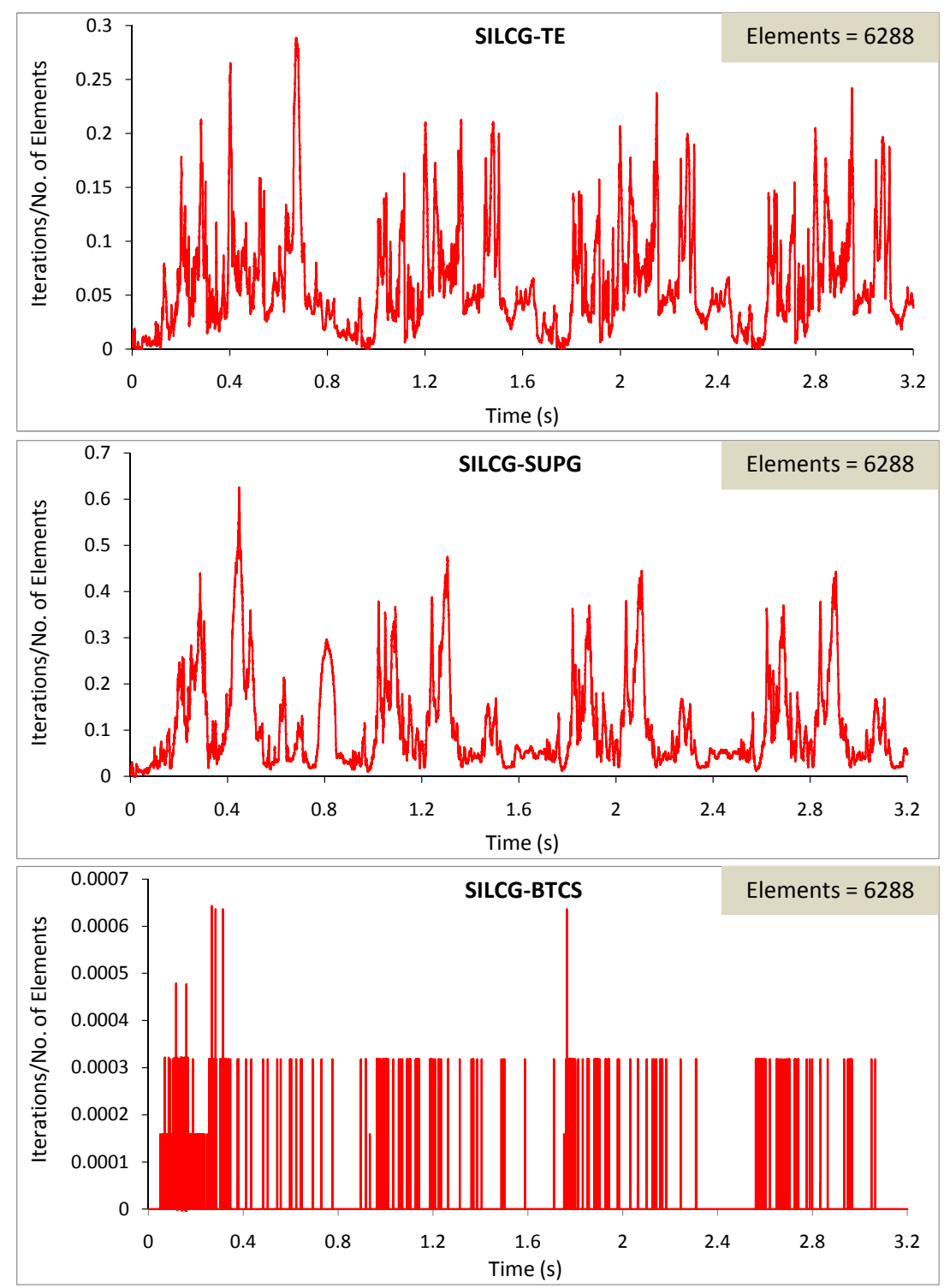

Figure 2.9: Number of iterations to number of elements ratio for for all three approaches. Jacobian matrix is calculated at every time step. 
Table 2.3: Averaged percentage $R M S E_{p}$ and $R M S E_{F}$ for all values shown in Table 2.2 for each method.

\begin{tabular}{lccc}
\hline Error $\%$ & SILCG-TE & SILCG-SUPG & SILCG-BTCS \\
\hline$R M S E_{p}$ & 0.1574 & 0.1594 & 0.1504 \\
$R M S E_{F}$ & 0.4313 & 0.4174 & 0.3248 \\
\hline
\end{tabular}
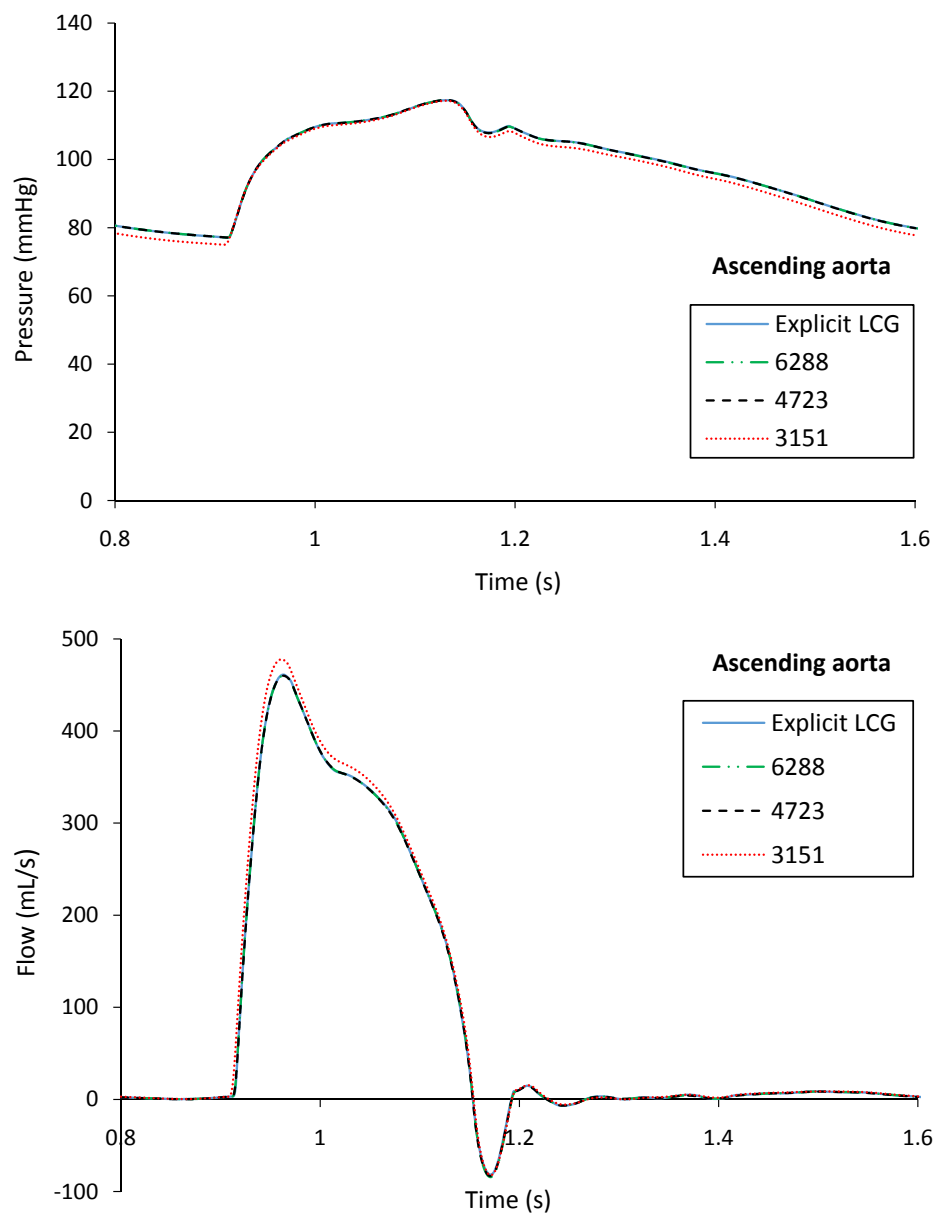

Figure 2.10: Mesh convergence of SILCG-TE method. 

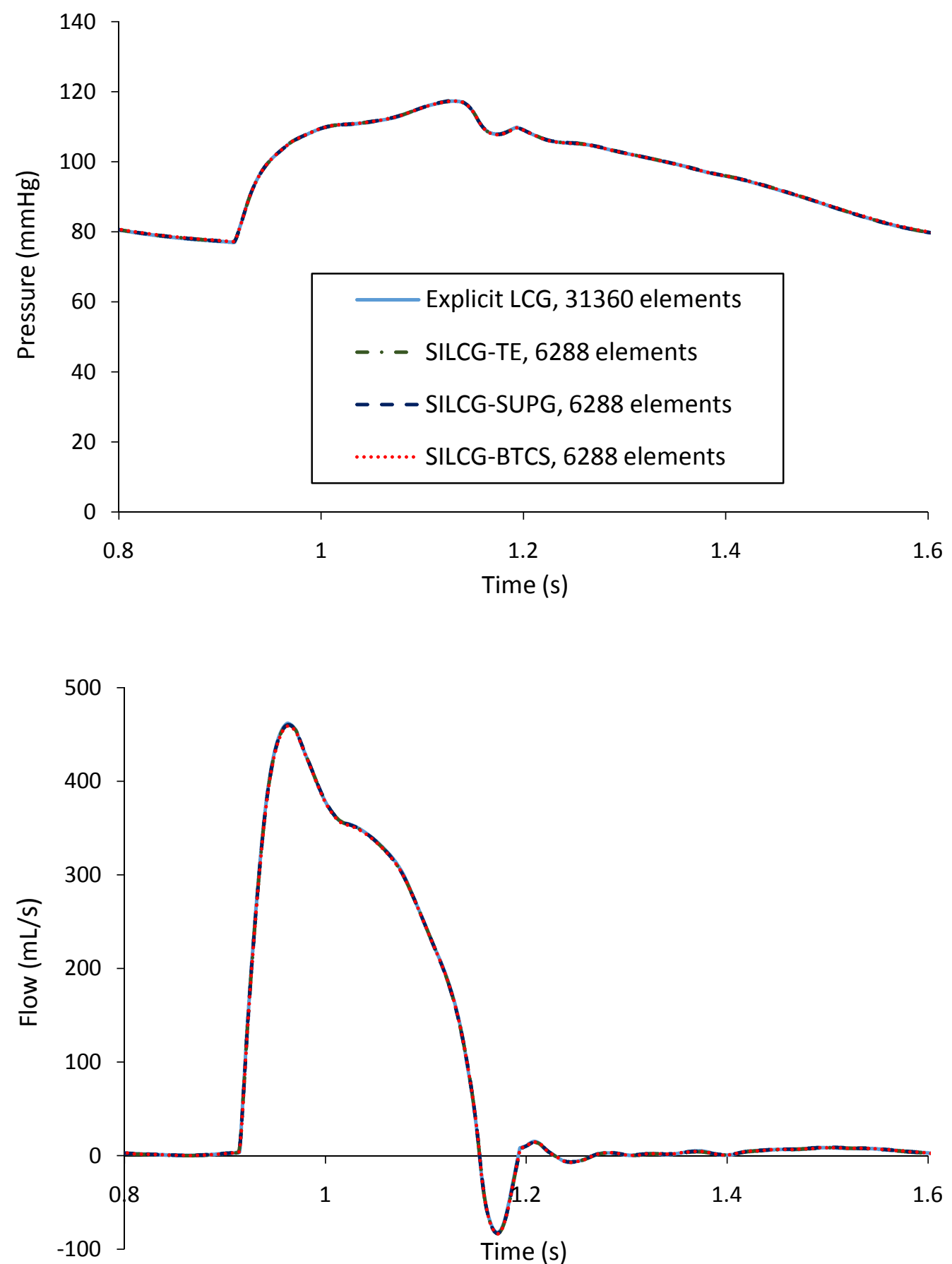

Figure 2.11: Pressure and flow variations in the ascending aorta obtained by the new methods against the original LCG using a fine mesh. 
Table 2.4: Computational speed CPU time per cardiac cycle, $l_{e}=0.25 \mathrm{~cm}$.

\begin{tabular}{ccccc}
\hline $\begin{array}{c}\text { Jacobian matrix } \\
\text { status }\end{array}$ & $\begin{array}{c}\text { SILCG-TE } \\
(\mathrm{min})\end{array}$ & $\begin{array}{c}\text { SILCG-SUPG } \\
(\mathrm{min})\end{array}$ & $\begin{array}{c}\text { SILCG-BTCS } \\
(\mathrm{min})\end{array}$ & $\begin{array}{c}\text { Explicit LCG } \\
(\mathrm{min})\end{array}$ \\
\hline case 1 & 29.63 & 33.19 & 3.40 & 4.32 \\
case 2 & 13.05 & 14.20 & 3.06 & 4.32 \\
\hline
\end{tabular}

\subsubsection{Computational efficiency}

Finally, we evaluate the CPU time for all proposed methods as shown in Table 2.4, by considering the maximum admissible time step sizes. The calculations are achieved on a processor of Intel(R) Core(TM) i5-4210U CPU @ 1.70GHz, 8GB RAM. The number of elements used is 6288, and the results are presented for two cases of Jacobian matrix calculations. In case 1, the Jacobian matrix is updated at every iteration, while for case 2, the matrix is only factorized once per time step. Table 2.4 shows that the SILCG-BTCS is the fastest one among the methods proposed due to the significant simplification in the numerical formulation discussed in subsection 2.2.2. For this reason, the CPU time is almost independent of the way in which Jacobian matrix is computed. For the other methods, continuous Jacobian matrix updating (case 1) significantly affects the computational performances. Overall, the maximum CPU time taken is less than 34 minutes and the minimum is just over 3 minutes per cardiac cycle.

\subsection{Conclusions}

A set of semi-implicit, locally conservative Galerkin methods (SILCG) have been proposed and tested for blood flow in a human arterial system. The three novel methods proposed include a Taylor expansion based method SILCG-TE, a streamline upwind Petrov- 
Galerkin SILCG-SUPG method and backward in time and central in space SILCG-BTCS type method. Although the SILCG-BTCS approach is the most straightforward and simple one, it is unstable beyond a time step $\Delta t=0.1 \mathrm{~ms}$. This is not a major disadvantage as the method can be very fast without the additional stabilization terms. The other two methods can admit larger time steps but the accuracy may deteriorate with the increase in time step values. None of the new methods are unconditionally stable as the characteristic variables and interior boundary fluxes are used from the previous time step. Overall, the proposed semi-implicit methods represent a promising alternative to the existing explicit LCG method, as the time step is not restricted by the CFL condition. Among the three approaches, SILCG-BTCS is the best in terms of accuracy and speed. However, the other two techniques are stable at larger time steps. So, for the current kind of problems, SILCG-BTCS would be recommended and the rest can be used when a larger time step is required. Moreover, the proposed methodology has the advantage of avoiding large matrix assembly, as it requires the only factorization at the element level. Further research is required to make the proposed method fully implicit and more computationally efficient. 


\section{Chapter 3}

Modelling energy transport within human body 


\subsection{Introduction}

In this chapter, we shall develop a set of three novel semi-implicit locally conservative Galerkin methods, SILCG-TE, SILCG-SUPG and SILCG-BTCS for bioheat transfer in a human body. This is an extended work of the methods that previously implemented for flow [10]. The proposed works will be compared with the available data in the literature in order to demonstrate their accuracy and stability. Also, the computational performance will be tackled here. In section 3.2, the governing equations, numerical procedure, and boundary conditions will be discussed. Then, a detailed demonstration of the accuracy, robustness of the proposed methods will be proved in section 3.3. Finally, the conclusions are reported in section 3.4.

\subsection{Mathematical formulations}

As flow solution has been addressed in the previous chapter, we shall discuss solutions of energy equation and conduction that occur in a vessel wall. By considering the flexible tube shown in Figure 3.1, the energy balance can be written as [58]

$$
\begin{aligned}
\dot{\Phi}_{\text {conv }}(t)= & \dot{W}_{\text {wall }}(t)+\dot{W}_{\text {shear }}(t)+\dot{\Phi}_{\text {cond }}(0, t)-\dot{\Phi}_{\text {cond }}(l, t) \\
& +\rho Q(l, t)\left(E_{s}(l, t)+\frac{p(l, t)}{\rho}\right)-\rho Q(0, t)\left(E_{s}(0, t)+\frac{p(0, t)}{\rho}\right) \\
& +\rho \frac{\partial}{\partial t} \int_{0}^{l} A(x, t) E_{s}(x, t) d x,
\end{aligned}
$$

where $E_{s}$ refers to the specific energy of the fluid estimated as a sum of the specific internal and kinetic energies $\left(E_{S}=e+\frac{u^{2}}{2}\right) . \dot{\Phi}_{c o n d}$ represents conduction fluxe in the fluid, $\dot{\Phi}_{\text {conv }}$, $\dot{W}_{\text {wall }}$ and $\dot{W}_{\text {shear }}$ are respectively the thermal flux in which exchanged by convection, integral quantities due to fluid effects on the tube walls and viscous forces. The simplified form of the transient one dimensional energy conservation equation may be given (viscous effects are neglected) as [58] 


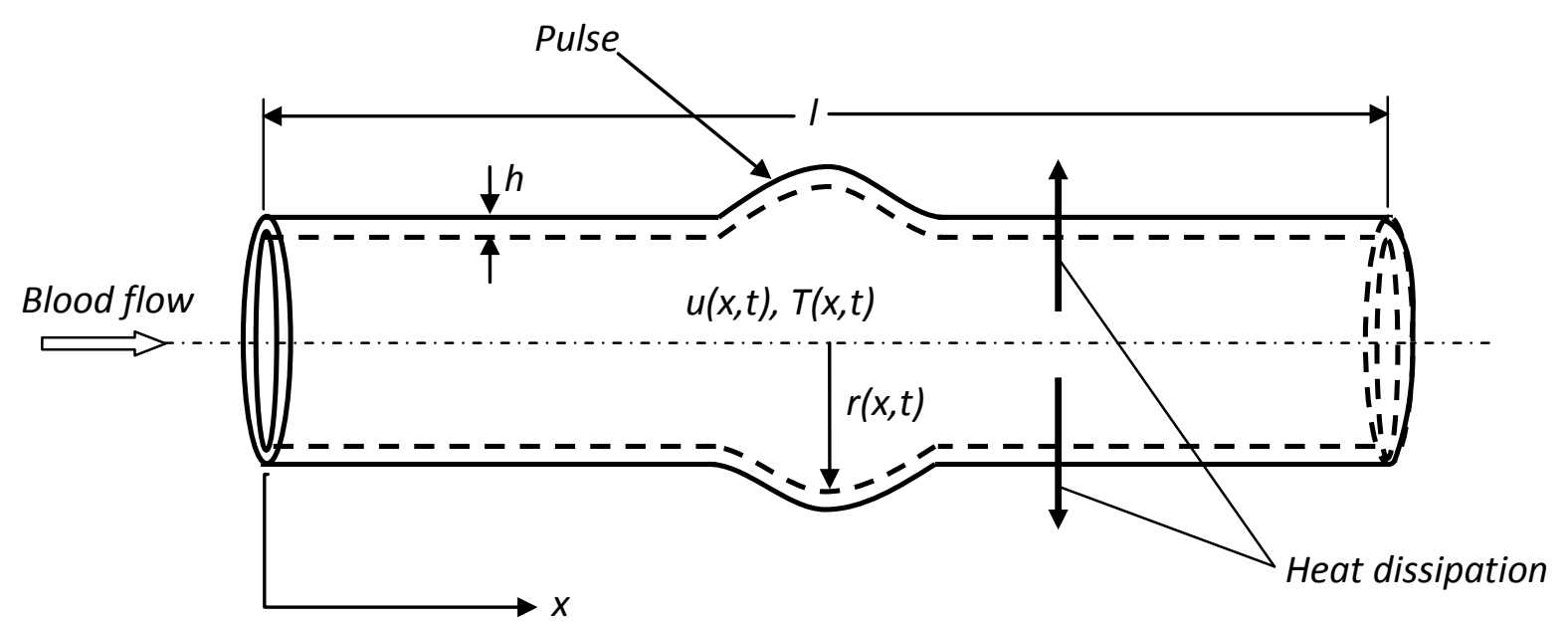

Figure 3.1: Schematic representation of arterial segment.

$$
\frac{\partial T}{\partial t}=\frac{\partial G}{\partial x}+\frac{2 h_{i n}}{\rho c_{p} \sqrt{A / \pi}}\left(T_{t, i n}-T\right) .
$$

The flux $G$ is expressed by

$$
G=-u T+\alpha_{f} \frac{\partial T}{\partial x}
$$

where $\alpha_{f}$ is the thermal diffusivity of the blood, $h_{i n}$ is the convective heat transfer coefficient for the inner surface of the vessel and $T_{t, i n}$ is the inner surface temperature. Also, to estimate heat transferred between the fluid and the surrounding environment, the transient polar form of the one dimensional heat conduction governing equation in the radial orientation is considered, i.e. [114]

$$
\rho_{t} c_{p, t} \frac{\partial T_{t}}{\partial t}-k_{t} \frac{1}{r} \frac{\partial}{\partial r}\left(r \frac{\partial T_{t}}{\partial r}\right)=q_{m}+\phi \rho c_{p}\left(T-T_{t}\right),
$$

where $r$ is the radial coordinate and $\rho_{t}, c_{p_{t}}, k_{t}$ are respectively the density, specific heat and thermal conductivity of the tissues material. $q_{m}$ is the volumetric heat generation or the metabolism term which mainly represents the energy due to the biological process and $\phi$ is tissue perfusion coefficient. 


\subsubsection{Numerical methods}

Applying same approaches presented in the previous chapter to the energy conservation given in Eq. (3.2), the numerical schemes can be divided into three categories as shown below.

\section{SILCG-TE}

Using Taylor expansion based method similar to the procedure applied to the system shown in Eq. (2.8) [12, 39, 40], differentiating Eq. (3.2) with respect to time and applying the chain rule gives

$$
\frac{\partial^{2} T}{\partial t^{2}}=-u \frac{\partial}{\partial x} \frac{\partial T}{\partial t}+\alpha \frac{\partial^{2}}{\partial x^{2}} \frac{\partial T}{\partial t}+Q \frac{\partial T}{\partial t}
$$

where

$$
Q=\frac{\partial D}{\partial T}=\frac{\partial}{\partial T}\left(\frac{2 h_{i n}}{\rho c_{p} \sqrt{A / \pi}}\left(T_{t, i n}-T\right)\right) .
$$

Rewriting Eq. (2.9) in terms of $T$ generates

$$
T^{n+1}=T^{n}+\Delta t{\frac{\partial T^{n+\theta}}{\partial t}}^{n+\frac{\Delta t^{2}}{2}}{\frac{\partial^{2} T^{n+\theta}}{\partial t^{2}}}^{n+}
$$

Now substituting Eqs. (3.2) and (3.5) into Eq. (3.7), the semi-discrete form reads

$$
\frac{T^{n+1}-T^{n}}{\Delta t}=\left\{-u \frac{\partial T}{\partial x}+\alpha \frac{\partial^{2} T}{\partial^{2} x}+D+\frac{\Delta t}{2}\left[u^{2} \frac{\partial^{2} T}{\partial^{2} x}-2 u Q \frac{\partial T}{\partial x}+2 \alpha Q \frac{\partial^{2} T}{\partial^{2} x}+Q D\right]\right\}^{n+\theta}
$$

In above equation, the higher order terms are omitted. Here, $\theta$ also varies from 1 to 0 . Applying linear finite elements procedure to the Eq. (3.8) generates [97]

$$
\begin{gathered}
\int_{\Omega} N \frac{T^{n+1}-T^{n}}{\Delta t} d \Omega=\int_{\Omega} N\left\{-u \frac{\partial T}{\partial x}+\alpha \frac{\partial^{2} T}{\partial^{2} x}+D\right\}^{n+\theta} d \Omega \\
+\left\{\frac{\Delta t}{2}\left[u^{2} \frac{\partial^{2} T}{\partial^{2} x}-2 u Q \frac{\partial T}{\partial x}+2 \alpha Q \frac{\partial^{2} T}{\partial^{2} x}+Q D\right]\right\}^{n+\theta} d \Omega
\end{gathered}
$$


The approximated solution for temperature field is expressed as

$$
T=\mathbf{N} \tilde{\mathbf{T}}
$$

where

$$
\mathbf{N}=\left[\begin{array}{llll}
N_{1} & N_{2} & \ldots & N_{m}
\end{array}\right]^{T}, \quad \tilde{\mathbf{T}}=\left[\begin{array}{llll}
\tilde{T}_{1} & \tilde{T}_{2} & \ldots & \tilde{T}_{m}
\end{array}\right]
$$

The solution based on stabilized locally conservative Galerkin method (LCG) to Eq. (3.9) $[97,98,99]$ is determined as

$$
\left[\mathbf{M}_{e}\right]\{\Delta \tilde{\mathbf{T}}\}^{n}-\Delta t\left\{\left(\left[\mathbf{K}_{e}\right]+\left[\mathbf{A}_{e}\right]\right)\{\tilde{\mathbf{T}}\}^{n+1}+\left[\mathbf{L}_{e}\right]\{\mathbf{D}\}^{n+1}+\mathbf{q}_{\Gamma_{e}}^{n}\right\}=0
$$

Above matrices are $2 \times 2$ because there is only one variable per node. So, each element has $T_{1}$ and $T_{2}$ refering to the starting and end nodes (more details about LCG for convection diffusion problem can be found in [99]). The term $\mathbf{q}_{\Gamma_{e}}$ is the numerical fluxes exchanged between adjacent elements which may be defined as given in Eq. (3.3) [97, 98, 99]

$$
\mathbf{q}_{\Gamma_{e}}=n\left(\tilde{u} \tilde{T}-\alpha \frac{\partial \tilde{T}}{\partial x}\right)
$$

where $n$ is the outward normal from the elements edges (see [99] for more details). This flux is treated at $n$ time level. The unique nodal solution for the node $i$ (see Figure 2.1) is obtained by the averaging each two values obtained at a nodes as

$$
\tilde{T}_{i}=\frac{\tilde{T}_{i e 1}+\tilde{T}_{i e 2}}{2}
$$

\section{SILCG-SUPG}

Similar steps applied to the flow in Eqs. (2.23) and (2.24) are also applied here. The modified shape function $\mathbf{W}$ and optimal $\alpha$ are reformulated according to [108]. The rest of the numerical procedure is identical to that of the SILCG-TE method discussed above. 


\section{SILCG-BTCS}

Again, SILCG-BTCS is the most simplified approach because it deals with the original governing equations. Here, all stabilization terms that come from Taylor expansion in SILCG-TE and from streamline upwind Petrov-Galerkin in SILCG-SUPG are removed. The rest of the numerical procedure follows as discussed for flow case, i.e. discretizing the original governing equation given in Eq. (3.2).

\subsubsection{Solution algorithm}

The solution of the discrete system defined in Eq. (3.12) is obtained by employing the Newton's method $[108,110]$. The steps shown in subsection 2.2.3 are reformulated to calculate the temperature $\tilde{\mathbf{T}}=\left[\begin{array}{ll}\tilde{T}_{1} & \tilde{T}_{2}\end{array}\right]^{T}$ at the iteration $k+1$ as

$$
\tilde{\mathbf{T}}^{k+1}=\tilde{\mathbf{T}}^{k}+\delta \tilde{\mathbf{T}}^{k}
$$

The update $\delta \tilde{\mathbf{T}}^{k}$ is estimated via

$$
\mathbf{J}\left(\tilde{\mathbf{T}}^{k}\right) \delta \tilde{\mathbf{T}}^{k}=-\mathbf{R}\left(\tilde{\mathbf{T}}^{k}\right)
$$

where Jacobian matrix $\mathbf{J}\left(\tilde{\mathbf{T}}^{k}\right)$ is a $2 \times 2$ here. The detailed presentation of the above equation including the residual vector is $\mathbf{R}\left(\tilde{\mathbf{T}}^{k}\right)$ given as

$$
\left[\begin{array}{ll}
\frac{\partial R_{T_{1}}}{\partial T_{1}} & \frac{\partial R_{T_{1}}}{\partial T_{2}} \\
\frac{\partial R_{T_{2}}}{\partial T_{1}} & \frac{\partial R_{T_{2}}}{\partial T_{2}}
\end{array}\right]\left\{\begin{array}{c}
\delta \tilde{T}_{1} \\
\delta \tilde{T}_{2}
\end{array}\right\}=-\left\{\begin{array}{l}
R_{T_{1}} \\
R_{T_{2}}
\end{array}\right\} .
$$

Above equation is solved iteratively with a proper initial guess at the first iteration. After that, updating process continues from the previous iteration until convergence. The LU factorisation [112] is adopted for solving the linear system of equation presented above. The solution is estimated at each time step according to the following algorithm 
Algorithm 2

1. do $\mathrm{i}=1, \mathrm{~m} \quad \mathrm{~m}=1,2, \ldots \ldots$,elements

2. do $\mathrm{j}=1,2 \quad$ nodes

3. initialise $\tilde{\mathbf{U}}^{k, n+1}=\tilde{\mathbf{U}}^{k, n}$ and $\tilde{\mathbf{T}}^{k, n+1}=\tilde{\mathbf{T}}^{k, n}$

4. end do

5. do $\mathrm{k}=1$,number of iterations (flow solution)

6. call Algorithm 1 (steps from 6 to 10)

7. if step 10 is satisfied $\rightarrow$ go to the next step

8. do $\mathrm{k}=1$,number of iterations (temperature solution)

9. compute $\mathbf{J}\left(\tilde{\mathbf{T}}^{k}\right)$ and $\mathbf{R}\left(\tilde{\mathbf{T}}^{k}\right)$

10. call LU and solve for $\delta \tilde{\mathbf{T}}^{k}$ through Eq. (3.16)

11. evaluate, if $\delta \tilde{\mathbf{T}}^{k} \leq$ tolerance

yes $\rightarrow$ go step 13

no $\rightarrow$ put $\tilde{\mathbf{T}}^{k+1}=\tilde{\mathbf{T}}^{k}$

12. end do

13. update through Eq. (3.15)

14. end do

Apparently, flow solution (i.e. Algorithm 1) is consolidated with temperature solution procedures. The stopping tolerance for the algorithms is chosen to be equal to $10^{-8}$.

\subsubsection{Solid discretization}

The solution of heat conduction in the wall was already developed in [6] where the standard forward Euler method (not LCG) is used. The method uses the central difference scheme for spatial discretization and a first order discretization for the time term. Thus, 
the discrete form of Eq. (3.4) for a node $i$ may be written as [6]

$$
\begin{aligned}
& T_{t, i-1}^{n} \frac{\Delta t k_{t}}{\rho_{t} c_{p, t} r_{i}}\left(-\frac{r_{i}}{\Delta r^{2}}+\frac{1}{2 \Delta r}\right)+T_{t, i}^{n}\left(1+\frac{2 \Delta t k_{t}}{\rho_{t} c_{p, t} \Delta r^{2}}\right)-T_{t, i+1}^{n} \frac{\Delta t k_{t}}{\rho_{t} c_{p, t} r_{i}}\left(\frac{r_{i}}{\Delta r^{2}}+\frac{1}{2 \Delta r}\right) \\
& =\frac{\Delta t}{\rho_{t} c_{p, t}} q_{v}+T_{t, i}^{n-1}
\end{aligned}
$$

where $i$ refers to number of nodes as $i=1,2, . . m$. Since the matrix of the linear system is tridiagonal, Thomas algorithm is used to solve the above system [6].

\subsubsection{Initial and bondary conditions}

Appropriate initial and boundary conditions assigned with the discrete formula by employing the characteristics system $[115,103,58,6]$. So, simplifying the governing equation (i.e. Eq. (3.2) by assuming the diffusion and source terms equal to zero as

$$
\frac{\partial T}{\partial t}+u \frac{\partial T}{\partial x}=0
$$

Here, similar approach used in subsection 2.2.4 is applied to the above equation. But because there is only $u$ appears in the equation (i.e. no matrix), it is easy to specify that $\lambda_{\text {temp }}=u$. So, the characteristic variable is $w_{\text {temp }}=T[58,6]$. This illustrates that the temperature is a property transferred by flow with an effective velocity $u$. For the first time step an initial temperature field of $36.8^{\circ}$ is applied and then the characteristic variable $w_{\text {temp }}$ is estimated for the next time steps using a similar approach to other boundary conditions discussed in the previous chapter [6].

The Neumann boundary condition given below describes heat exchange between the body and the environment [6]

$$
-\left.k_{t} A_{t, \text { out }} \frac{\partial T_{t}}{\partial r}\right|_{r_{\text {out }}}=q_{\text {con }, \text { out }}+q_{\text {rad }}+q_{\text {eva }}
$$


where the left side of the obove equation represents $q_{\text {skin }}$ which is the sum of the convective flux $q_{\text {con,out }}$, radiation flux $q_{\text {rad }}$ and the evaporation losses is $q_{\text {eva }} . q_{\text {con,out }}$ and $q_{\text {rad }}$ are estimaated according to Fiala et al. [9] and expressed as

$$
q_{\text {con }, \text { out }}=h_{\text {con }, \text { out }}\left(T_{t}\left(r_{\text {out }}\right)-T_{\text {out }}\right),
$$

where $h_{\text {out }, \text { con }}$ is the convective heat transfer coefficient. The radiative flux exchanged is given by the following expression

$$
q_{\text {rad }}=h_{\text {rad }}\left(T_{t}\left(r_{\text {out }}\right)-T_{\text {sur }, m}\right)
$$

where $T_{\text {sur, }}$ is the mean surrounding temperature and $h_{\text {rad }}$ is the radiative heat coefficient. Above boundary conditions are evaluated after achieving the solution for the temperature field within blood [6]. This will be discussed later in the solution algorithm.

\subsubsection{Arterial tree-solid tissues architecture}

The body is divided into fourteen multilayered cylinders representing respectively head, neck, shoulders, thorax, abdomen, arms, forearms, thighs and legs (details of the physical model are given in the Table 3.1). Each cylinder consists of bone, muscle, fat tissue and skin layers. Also, the inner organs, brain, lungs, and viscera in the head, thorax and abdomen respectively are included. Figure 3.2 shows the fourteen cylinders with the arterial tree. This configuration shows the embedded blood vessels through body tissues (the cylinders). These cylinders will be treated as asymmetric geometries around the arteries and the heat is transferred via radial direction. More specific details will be discussed in subsection 3.2.7. So, this three dimensional figure (manikin) is for illustration purposes only. 


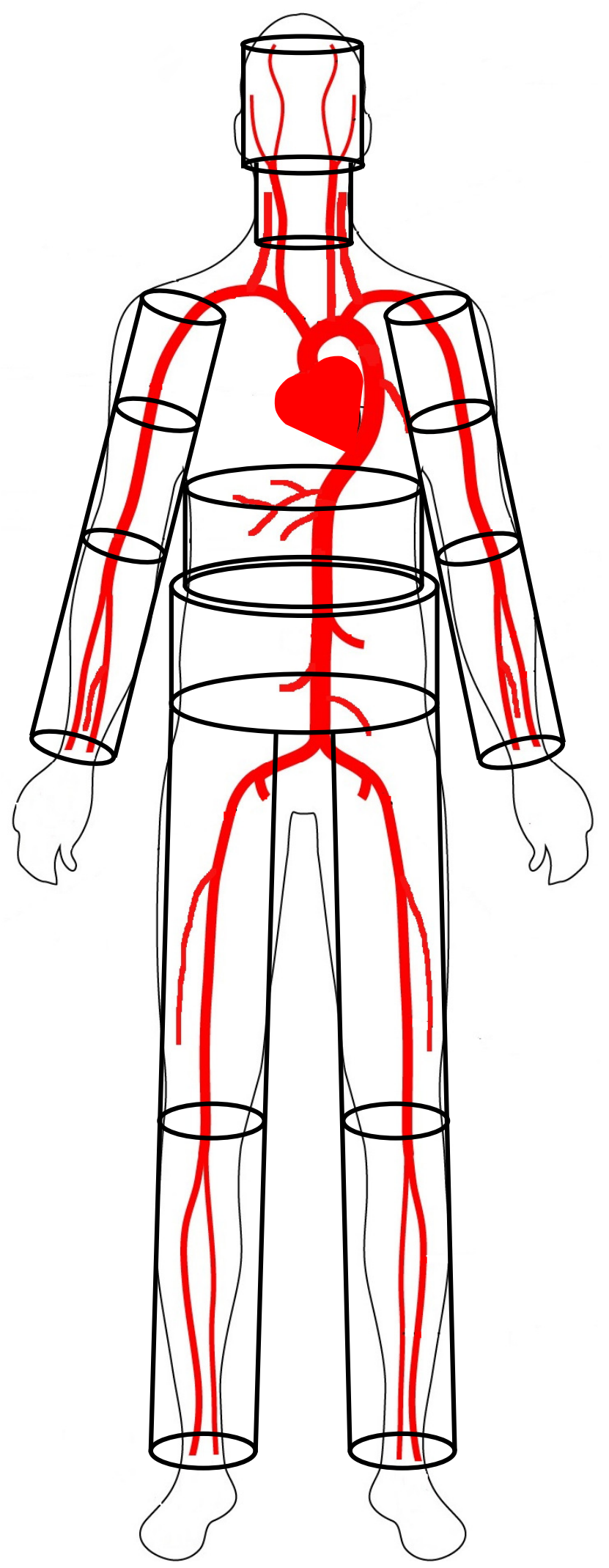

Figure 3.2: Manikin configuration of main arterial network with tissues (14 cylinders) in human body. 
Table 3.1: Tissue physical properties [6]

\begin{tabular}{cccc}
\hline Cylinder & Tissues & Layer radii $(\mathrm{cm})$ & Length $(\mathrm{cm})$ \\
\hline head & brain, bone, fat, skin & $6.6,7.6,7.8,8.0$ & 23.5 \\
neck & bone, muscle, fat, skin & $1.9,5.4,5.6,5.8$ & 7.9 \\
thorax & lung, bone, muscle, fat, skin & $7.7,8.9,12.3,12.612 .9$ & 15.6 \\
abdomen & viscera, bone, muscle, fat, skin & $7.9,8.3,10.9,12.4,12.6$ & 24.8 \\
shoulder & bone, muscle, fat, skin & $3.7,3.9,4.4,4.6$ & 13.4 \\
arm & bone, muscle, fat, skin & $1.5,3.4,4.0,4.2$ & 29.6 \\
forearm & bone, muscle, fat, skin & $1.5,3.4,4.0,4.2$ & 23.7 \\
thigh & bone, muscle, fat, skin & $2.2,4.8,5.3,5.5$ & 58.5 \\
leg & bone, muscle, fat, skin & $2.2,4.8,5.3,5.5$ & 34.3 \\
\hline
\end{tabular}

\subsubsection{Thermoregulatory System}

The thermo-neutrality case exists when the mean skin and core temperature of the body are respectively $33.7^{\circ} \mathrm{C}$ and $36.8^{\circ} \mathrm{C}$ [116]. The energy exchanges between the body and environment occurs when above temperature limits violated and thus the thermoregulatory system is stimulated to control the body homeostasis [6]. The thermoregulatory system activation is represented by three mechanisms, shivering (lower skin temperature), sweating (higher skin temperature) and vasomotion (flow control). The core Temperature $T_{\text {core }}$ is the mean temperature of the first layer of inner nodes sets of the head, neck, thorax, and abdomen. Skin temperature $T_{\text {skin }}$ is the averaged value of the skin temperature. The shivering mechanism $q_{\text {shiv }}$ can be evaluated by dividing the total sum of segmental heat generation by muscle volume [84]. The shivering temperature $T_{\text {shiv }}$ is a function of the core temperature, i.e. 


$$
\left\{\begin{array}{l}
T_{\text {shiv }}=35.5^{\circ} \mathrm{C} \text { if } T_{\text {core }}<35.8{ }^{\circ} \mathrm{C} \\
T_{\text {shiv }}=-10222+570.9 T_{\text {core }}-7.9455 T_{\text {core }}^{2}\left({ }^{o} \mathrm{C}\right) \\
\text { if } 35.8{ }^{\circ} \mathrm{C} \leq T_{\text {core }} \leq 37.1{ }^{\circ} \mathrm{C}
\end{array}\right.
$$

The ultimate increase of the total metabolic heat rate caused by shivering $Q_{\text {shiv, } \max }$ may be given as

$$
Q_{\text {shiv }, \max }=\frac{1}{3600}\left(-1.186110^{9}+6.55210^{7} T_{\text {core }}-9.041810^{5} T_{\text {core }}^{2}\right)(W) .
$$

If $T_{\text {core }}>37.1{ }^{\circ} \mathrm{C}$, shivering does not occurs. The shivering metabolic heat generation $Q_{\text {shiv }}$ may now be estimated as [6]

$$
Q_{\text {shiv }}=Q_{\text {shiv, } \max }\left[1-\left(\frac{T_{\text {skin }}-20}{T_{\text {shiv }}-20}\right)^{2}\right](W) \text { if }\left(40-T_{\text {shiv }}\right) \leq T_{\text {skin }} \leq T_{\text {shiv }}\left({ }^{o} C\right) .
$$

Sweating limit $T_{\text {swe }}$ is approximated as a mean skin temperature given as [117]

$$
\left\{\begin{array}{l}
T_{\text {swe }}=42.084-0.15833 T_{\text {skin }}\left({ }^{\circ} \mathrm{C}\right) \text { if } T_{\text {skin }} \leq 33.0{ }^{\circ} \mathrm{C} \\
T_{\text {swe }}=36.85{ }^{\circ} \mathrm{C} \text { if } T_{\text {skin }}>33.0{ }^{\circ} \mathrm{C}
\end{array}\right.
$$

The sweat rate $\dot{m}_{\text {swe }}$ can be evaluated as

$$
\dot{m}_{\text {swe }}=\frac{45.8+739.4\left(T_{\text {core }}-T_{\text {swe }}\right)}{3.610^{6}}(\mathrm{~kg} / \mathrm{s}) \text { if } T_{\text {core }}>T_{\text {swe }}
$$

Relative skin wetness $w$ can be written as

$$
w=0.06+\frac{\dot{m}_{\text {swe }}(1-0.06)}{0.000193}
$$

The total evaporation heat loss $q_{\text {swe }}$ may now be written as [117]

$$
q_{\text {swe }}=\frac{w\left(P_{\text {skin }}-P_{\text {out }}\right)}{R_{\text {swe }, c l}+\frac{1}{f_{c l} h_{\text {swe }}}}\left(W / m^{2}\right)
$$


where $P_{\text {skin }}$ is the pressure of water vapour on skin, $R_{\text {swe,cl }}$ refers to evaporative heat resistance of the clothes layer, $f_{c l}$ is clothes area factor (defined as the surface area of the clothed body divided by the area of the unclothed body), and $h_{s w e}$ is the evaporation heat transfer coefficient.

Also, vasodilation and vasoconstriction mechanisms are included. They are respectively defined as an increase and decrease in arterial flow in the skin layers. At thermoneutrality conditions, flow considers a basal value $\dot{m}_{\text {skin,bas. }}$. If core temperature rises over the neutral value, vasodilation takes place. When core temperature records $37.2{ }^{\circ} \mathrm{C}$, the maximum flow is reached in the skin layer (i.e. $\dot{m}_{\text {skin,max }}$ ). If core temperatures are between $36.8{ }^{\circ} \mathrm{C}$ and $37.2{ }^{\circ} \mathrm{C}$, skin blood flow will follow core temperature linearly. As mean skin temperature is below the neutral value of $33.7^{\circ} \mathrm{C}$, vasoconstriction occurs. The maximum vasoconstriction is observed at a mean skin temperature equal to $10.7{ }^{\circ} \mathrm{C}$. At this temperature the skin blood flow assumes a minimum value $\left(\dot{m}_{s k i n, m i n}\right)$. Between skin temperatures of $33.7^{\circ} \mathrm{C}$ and $10.7^{\circ} \mathrm{C}$, skin blood flow is assumed to vary linearly with temperature.

The evaluation of above processes, i.e. vasodilation and vasoconstriction flows $\dot{m}_{\text {skin,dil }}$ and $\dot{m}_{s k i n, c o n}$ may be expressed as

$$
\left\{\begin{array}{l}
\dot{m}_{\text {skin,dil }}=\dot{m}_{\text {skin,bas }}(\mathrm{kg} / \mathrm{s}) \text { if } T_{\text {core }}<36.8^{\circ} \mathrm{C} \\
\dot{m}_{\text {skin,dil }}=\frac{T_{\text {core }}-36.8}{37.2-36.8}\left(\dot{m}_{\text {skin,max }}-\dot{m}_{\text {skin,bas }}\right) \\
+\dot{m}_{\text {skin,bas }}(\mathrm{kg} / \mathrm{s}) \text { if } 36.8^{\circ} \mathrm{C} \leq T_{\text {core }} \leq 37.2^{\circ} \mathrm{C} \\
\dot{m}_{\text {skin,dil }}=\dot{m}_{\text {skin,max }}(\mathrm{kg} / \mathrm{s}) \text { if } T_{\text {core }}>37.2^{\circ} \mathrm{C}
\end{array}\right.
$$


and

$$
\left\{\begin{array}{l}
\dot{m}_{\text {skin,con }}=\dot{m}_{\text {skin,min }}(\mathrm{kg} / \mathrm{s}) \text { if } T_{\text {skin }}<27.8^{\circ} \mathrm{C} \\
\dot{m}_{\text {skin,con }}=\frac{T_{\text {skin }}-27.8}{33.7-27.8}\left(\dot{m}_{\text {skin,bas }}-\dot{m}_{\text {skin,min }}\right) \\
+\dot{m}_{\text {skin,min }}(\mathrm{kg} / \mathrm{s}) \text { if } 27.8^{\circ} \mathrm{C} \leq T_{\text {skin }} \leq 33.7^{\circ} \mathrm{C} \\
\dot{m}_{\text {skin,con }}=\dot{m}_{\text {skin,bas }}(\mathrm{kg} / \mathrm{s}) \text { if } T_{\text {skin }}>33.7^{\circ} \mathrm{C}
\end{array}\right.
$$

The readers are refered to [6] for more details.

\subsubsection{Coupling blood with solid systems}

The coupling between blood vessels and surrounding tissues is important to obtain an acceptable solution. Here a one dimensional technique is considered as shown in Figure 3.3. This is a general configuration of the body cylinders. The arterial tree is divided into three categories, heart region, central and transversal arteries. The body is assumed to be axisymmetric and the nodes of the central artery (centered line) is assumed to coincide with the set of nodes of the first tisuue layer (first cylinder layer) as shown in the figure. So, each central node meets with a tissue node. This means there are longitudinal (central blood vessel) and radial (tissues cylinders) discretizations for the system but the one dimentional heat transfer is maintained. The solution is obtained by solving blood nodes (central longitudinal nodes) first. Once this is done, the calculations start for the tissue nodes (radial direction). The transversal vessels (dashed line) cross through one or more cylinders, so the volumetric source term $q_{m}$ is modified in Eq.(3.4) [6]. In the cylinders, head, neck, legs, and forearms there are transversal arteries only. Hence, along with the axis of the cylinders, an adiabatic condition is assumed. The inner heat transfer coefficient is set based on a constant Nusselt number is equal to 4 following to [118]. The respiration losses are considered by corporating a negative volumetric heat source $q_{b r e}$ at all lung nodes and given as [84] 


$$
q_{\text {bre }}=\frac{1}{V_{\text {lung }}}\left[0.0014 Q_{m, \text { glob }}\left(34-T_{\text {out }}\right)+0.0173 Q_{m, \text { glob }}\left(5.87-P_{\text {out }}\right)\right]\left(W / \mathrm{cm}^{3}\right)
$$

where $Q_{m, g l o b}$ is the general metabolic heat generation given as $58.2 \mathrm{~W} / \mathrm{m}^{2}$ and $V_{\text {lung }}$ refers to lungs volumes found to be $\left.5631.41 \mathrm{~cm}^{3}\right) . P_{\text {out }}$ is the water vapour pressure at the the ambient conditions. The fluid-solid coupling procedure is achieved as [6]

1. $T^{n+1}$ is calculated by using Algorithm 2 (i.e. by using all new technique).

2. Along the central arteries nodes (i.e. fluid nodes), $T^{n+1}$ is assumed to be equal to $T_{t}^{n}$ of the interacting tissue nodes.

3. $T_{\text {core }}$ and $T_{\text {skin }}$ are evaluated at $n$ level depending on $T_{t}^{n}$.

4. Contributions from transversal arteries, i.e. breathing, sweating and shivering are estimated using both $T^{n+1}$ and $T_{t}^{n}$.

5. $T_{t}^{n+1}$ is obtained implicitly using Eq. (3.18).

\subsection{Results and discussion}

As mentioned before, the physical model is a combination of the arterial tree developed by $[12,39]$ and the recent work of bioheat heat transfer model given by [6]. All physical data are given in those publications. We shall investigate the accuracy of the temperature field and the computational performance of the proposed schemes against the observations shown in $[6,84,87]$ and the experimental data given in $[52,96]$. Here, Algorithm 2 is used to compute $T$ for flow, and then this is coupled with $T_{t}$ as discussed in last five points in the previous subsection. The configuration shown in Figure 3.2 is solved to evaluate temperature and heat losses in different locations of the body. Time step for all proposed 


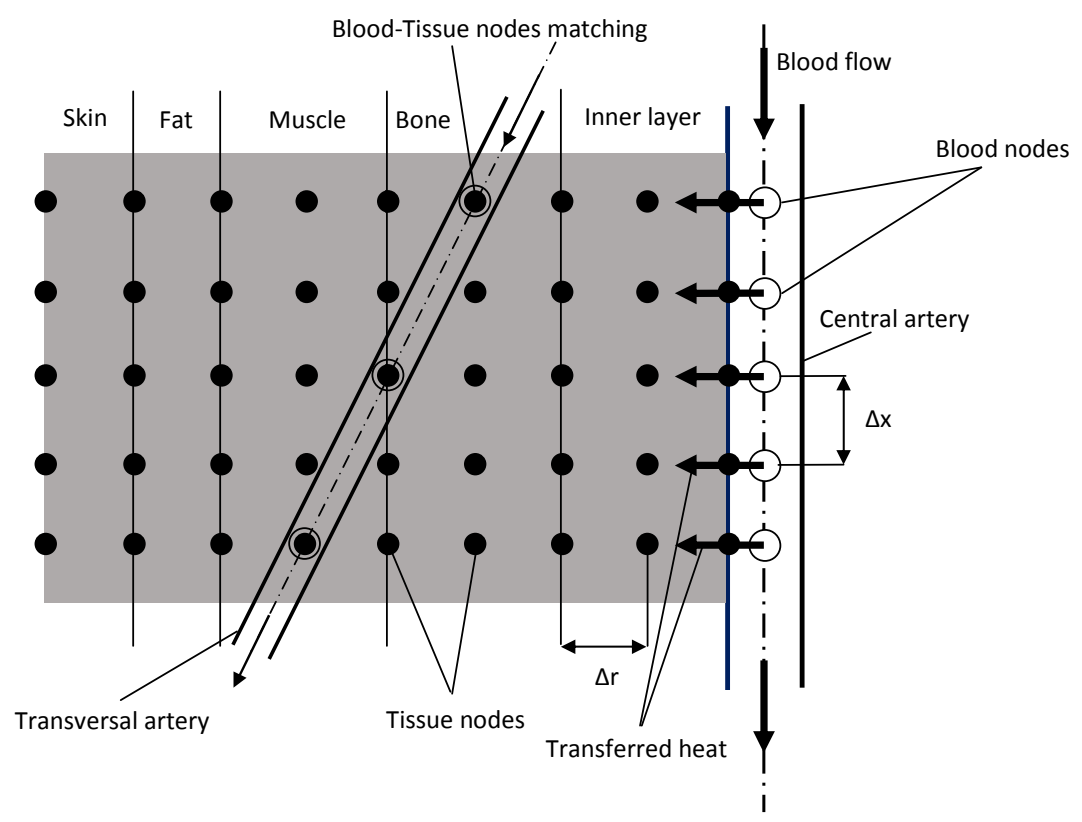

Figure 3.3: Coupling of an artery with tissue (represented by cylinders)

approaches is $0.1 \mathrm{~ms}$ and $l_{e}=0.25 \mathrm{~cm}$. It is convenient to recall CFL definition that is given as [40]

$$
\mathrm{CFL}=\frac{\lambda \Delta t}{l_{e}},
$$

where $\lambda$ is the maximum absolute velocity that refers to the characteristic speed in Eq. (2.29), $\Delta t$ and $l_{e}$ are respectively the time and spatial steps. This CLF number is considered although there is diffusion term (i.e. $\alpha_{f}$ ) as shown in Eq. (3.2) because the convection is dominant.

First, a bare body case is considered, thus $R_{s w e, c l}$ and $f_{c l}$ are equal to 0 and 1 respectively. Air velocity $v_{\text {air }}=4 \mathrm{~m} / \mathrm{min}$. Initial temperature field is set to be $36.8{ }^{\circ} \mathrm{C}$. Figure 3.4 shows a comparison between the new methods and the original explicit LCG developed in [6] and the experimental results shown in $[52,96]$ for the temperature in different locations, i.e. in tympanic and rectal points. Also, the evaporative heat losses (sweating and breathing latent losses) are evaluated. The body is exposed to three different external conditions (i.e. various external temperatures and relative humidity), $T_{\text {out }}=28.5{ }^{\circ} \mathrm{C}$ - 
$r \cdot h .=31 \%, T_{\text {out }}=17.7{ }^{\circ} \mathrm{C}-r \cdot h .=31 \%$ and $T_{\text {out }}=13.0{ }^{\circ} \mathrm{C}-r \cdot h .=45 \%$ as shown. Rectal temperature is monitored at the tissue node at an axial distance of $22 \mathrm{~cm}$ from the top of the abdominal cylinder and at a radius of $r=3.5 \mathrm{~cm}$. The tympanic site is assumed to be at a distance of $12 \mathrm{~cm}$ from the bottom of the head cylinder and at $r=5.0 \mathrm{~cm}$. The evaporative heat losses are calculated by adding the contributions of each cylinder section and then dividing by the total skin surface. The results prove that the proposed methods are stable and generating accurate outcomes. With respect to the results of [6], the predictions are almost identical. However, it can be noticed that there are some deviations against experimental data. These errors significantly decrease with time and reach nearly 0.25 ${ }^{o} \mathrm{C}$.

We also check the results for a longer time in Figure 3.5 for both core and skin temperatures (i.e. $T_{\text {core }}$ and $T_{\text {skin }}$ ). The body here is subjected to heat for 1 hour at $T_{\text {out }}=28.1^{\circ} \mathrm{C}$ - r.h. $=43 \%, 2$ hours at $T_{\text {out }}=47.8^{\circ} \mathrm{C}-r \cdot h .=27 \%$ and 1 hour at $T_{\text {out }}=28.3{ }^{\circ} \mathrm{C}-r \cdot h .=44 \%$. The results are compared with the experimental data shown in $[52,96]$ and the numerical ones given by $[6,84]$. Again, the present results closely agree with other results.

The behaviour of core temperature $T_{\text {core }}$ at cold external condition of $T_{\text {out }}=13{ }^{\circ} \mathrm{C}$ $r . h .=45 \%$ is reported in Figure 3.6. The findings are evaluated against same experimental data above and against the numerical data published by $[6,87]$. Two cases are reported by [87], one is for the body and the other one is plotted for the upper chest only. As can be seen, a good agreement with the established results is achieved for all new approaches.

Also, the results are plotted for blood temperature in three different locations and under various outside conditions, i.e. $T_{\text {out }}=28.5^{\circ} \mathrm{C}-r \cdot h .=31 \%, T_{\text {out }}=17.7{ }^{\circ} \mathrm{C}-r \cdot h .=31 \%$ and $T_{\text {out }}=13.0^{\circ} \mathrm{C}-$ r.h. $=45 \%$ as shown in Figure 3.7. The simulations are carried out for abdominal aorta, right external iliac and right radial arteries (respectively segments 43,58 and 16 shown in Figure 2.5) using SILCG-TE approach. Since the nature of the flow is pulsatile, the temperature behaves in a similar manner and also the blood vessels elasticity leads to temperature partially to be reflected like flow. In general, the blood temperature 


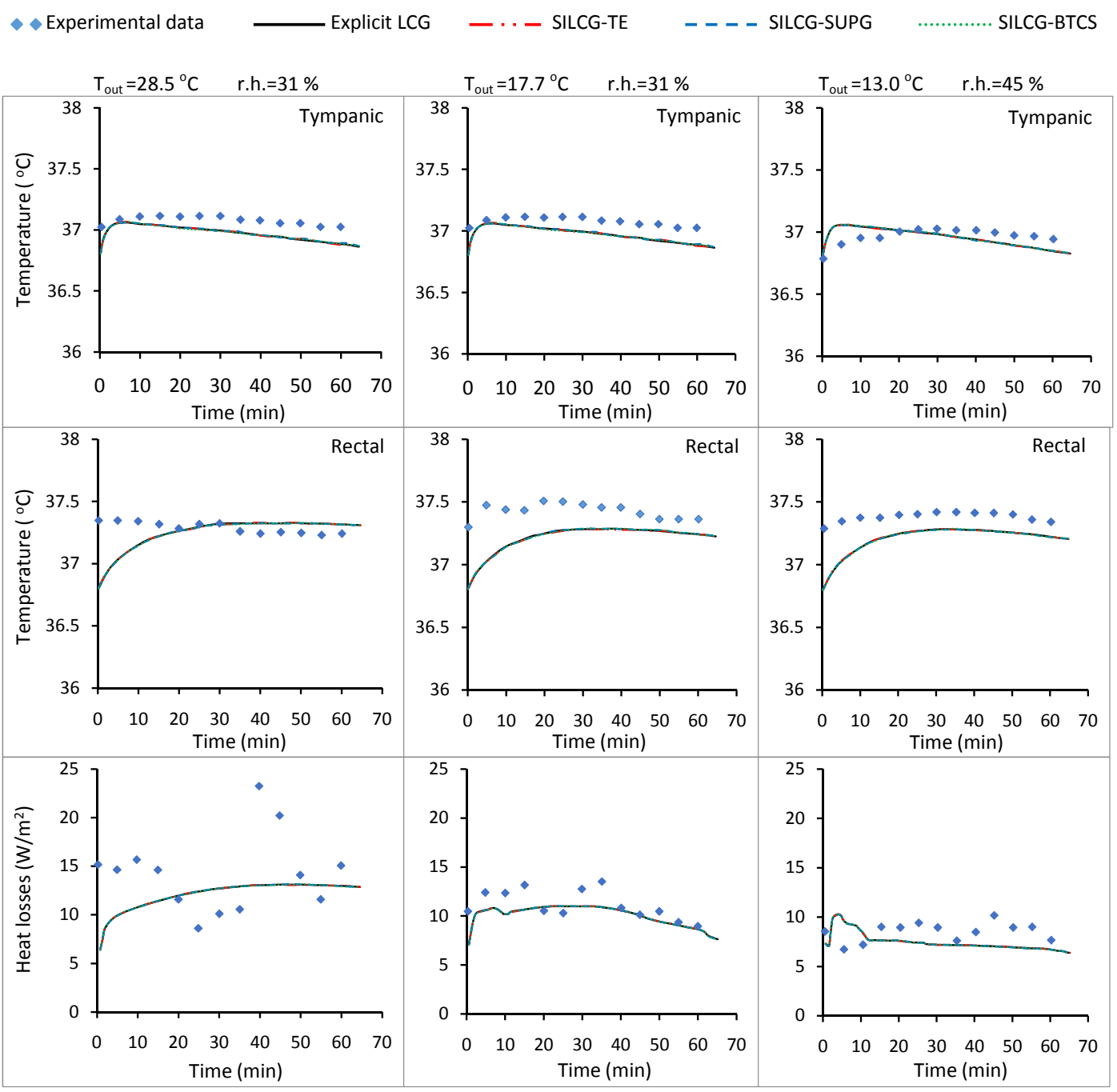

Figure 3.4: Tympanic, rectal temperatures and heat losses at different external conditions.

magnitude changes mildly for different atmospheric conditions. The distinguished temperature changes of the radial artery with the atmosphere is due to the small diameter and the non presence of the other mechanisms like metabolic activity [6].

In the following three figures, we investigate the behavior of iterations/no. of elements ratio for heat loop alone in Figure 3.8, and then the total cost in Figure 3.9. The latter figure is the sum of both flow and heat costs. Here, SILCG-BTCS is still the fastest one. In both figures above, Jacobian matrix is factorized at every single iteration (i.e. classical 
$\downarrow$ Experimental data

- Smith Model

— Explicit LCG $\quad$ - - - SILCG-TE

---- SILCG-SUPG

SILCG-BTCS
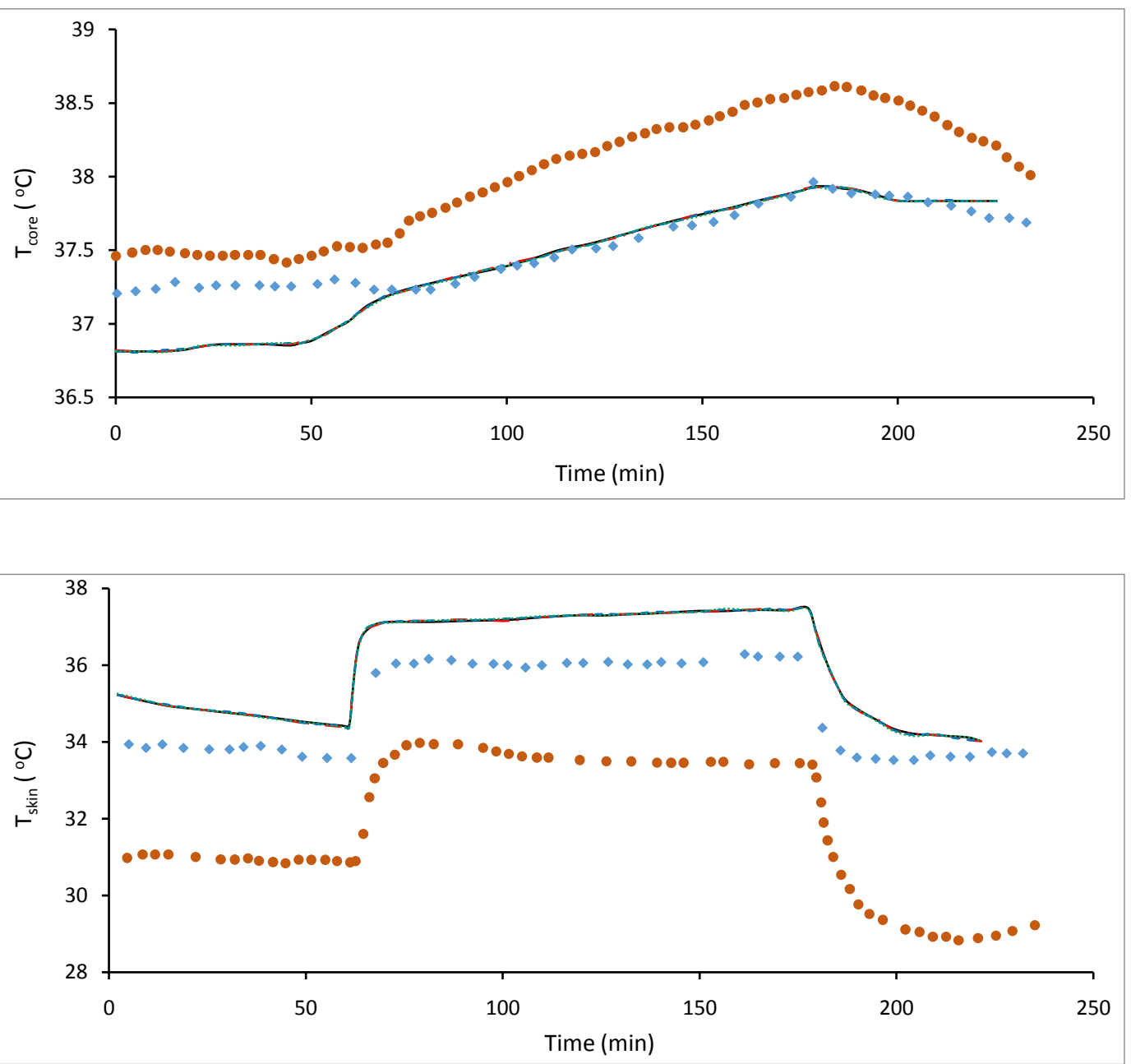

Figure 3.5: Core and skin temperature variations in a bare body under hot exposure.

Newton method). The experience shows here, it is possible to limit Jacobian matrix to one solution per each time step. This has been proved for the flow in the previous chapter. This significantly reduces computational time demands for the schemes especially SILCG-TE and SILCG-SUPG. For both methods, the additional stabilizer terms are expensive to estimate at each iteration. Figure 3.10 shows the total cost for both flow and temperature which is almost unaffected as a result of one Jacobian matrix solution per time step. 


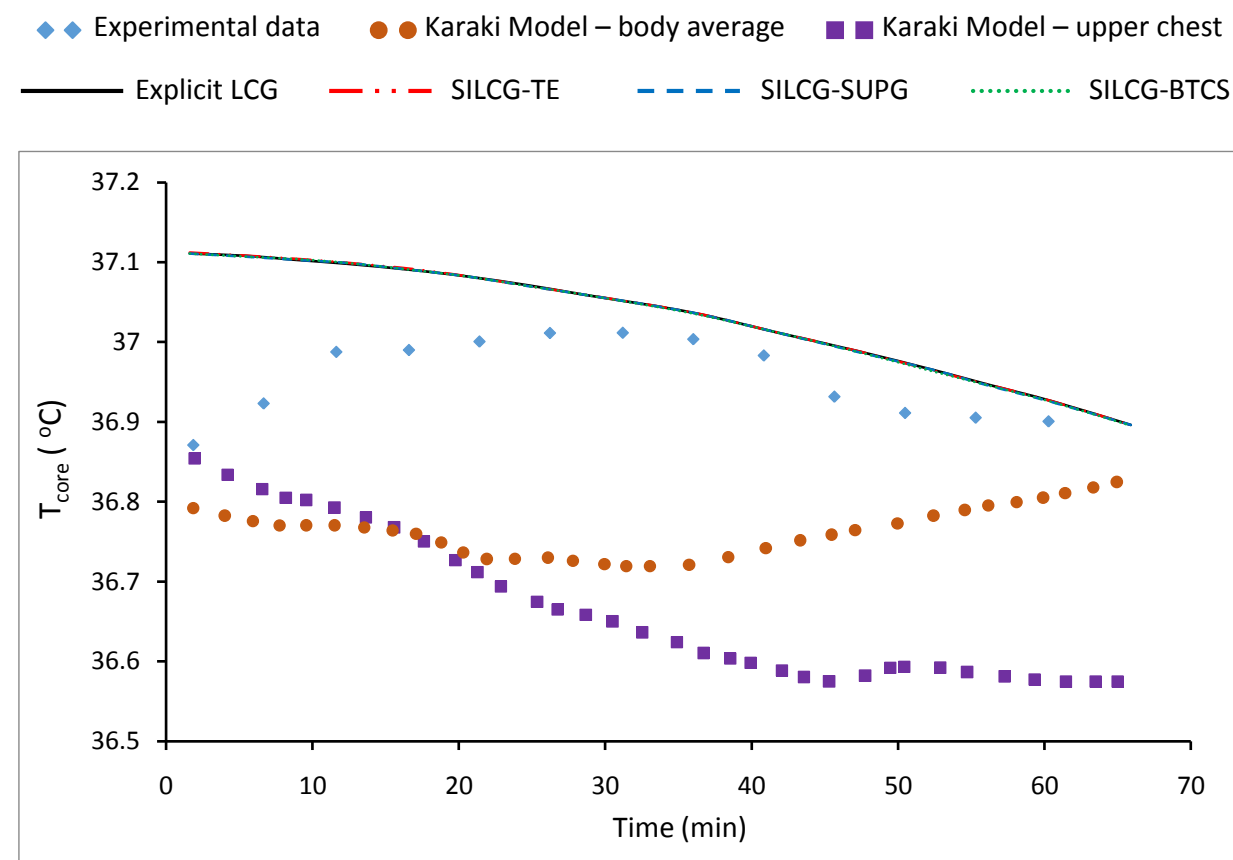

Figure 3.6: Core temperature variations in a bare body under cold exposure.

As both the velocity $u$ and area $A$ influence the temperature distribution in flexible tubes of the arterial tree, we attempt to investigate in depth the variations of both variables above as shown in Figures 3.11 and 3.12 respectively. The monitoring points are set in three different locations, i.e. right carotid, right subclavian II and right femoral (segments 13, 15 and 60 respectively.). As expected, all new techniques agree very well with reference data estimated by explicit LCG.

Also, the computational time is investigated here for all methods, including our reference approach (i.e. explicit LCG). The simulations are carried out on the clustered machine (Intel(R) Xeon(R) CPU E5-2680 v4 @ 2.40GHz, 64GB RAM) in Collage of Engineering, Swansea University. Again, case 1 refers to solving Jacobian matrix at every iteration while case 2 is for one solution at each time step. Similar to the case of flow, SILCG-BTCS is the faster due to the simplified approach. 
Table 3.2: Computational speed CPU time per cardiac cycle, $l_{e}=0.25 \mathrm{~cm}$.

\begin{tabular}{ccccc}
\hline $\begin{array}{c}\text { Jacobian matrix } \\
\text { status }\end{array}$ & $\begin{array}{c}\text { SILCG-TE } \\
(\mathrm{min})\end{array}$ & $\begin{array}{c}\text { SILCG-SUPG } \\
(\mathrm{min})\end{array}$ & $\begin{array}{c}\text { SILCG-BTCS } \\
(\mathrm{min})\end{array}$ & $\begin{array}{c}\text { Explicit LCG } \\
(\mathrm{min})\end{array}$ \\
\hline case 1 & 2.59 & 2.88 & 1.82 & 2.01 \\
case 2 & 2.23 & 2.47 & 1.80 & 2.01 \\
\hline
\end{tabular}

\subsection{Conclusions}

A novel set of three approaches have been developed for solving the energy conservation equation which governs temperature distribution within a human body. These methods include a Taylor expansion based method SILCG-TE, a streamline upwind Petrov-Galerkin method SILCG-SUPG and a backward in time and central in space SILCG-BTCS type method. The work here is an extension of flow solvers developed in the previous chapter. The proposed methods have demonstrated very good accuracy against the original explicit LCG and an acceptable agreement with other numerical and experimental data. Also, the computational performance has been shown using iterations/no. of elements ratio and among the techniques proposed, SILCG-BTCS is the fastest one. This is due to the removal of any additional stabilization terms that are associated with other methods. This is also reflected in the time consumed for each approach. Moreover, the experience demonstrates that Jacobian matrix solution can be limited to once per time step. This considerably saves time that is spent on the classical Newton procedure. Also, none of the methods are unconditionally stable because of the characteristic variables used for prescribing the boundary conditions and interior boundary fluxes over element edges are used from the previous time step. In general, the family of the new methods represents another tool along with the original explicit LCG. Also, the new approaches allow one to avoid large matrix assembly, as they require only an elementary level matrix which is $2 \mathrm{x}$ 
2 in this case. 

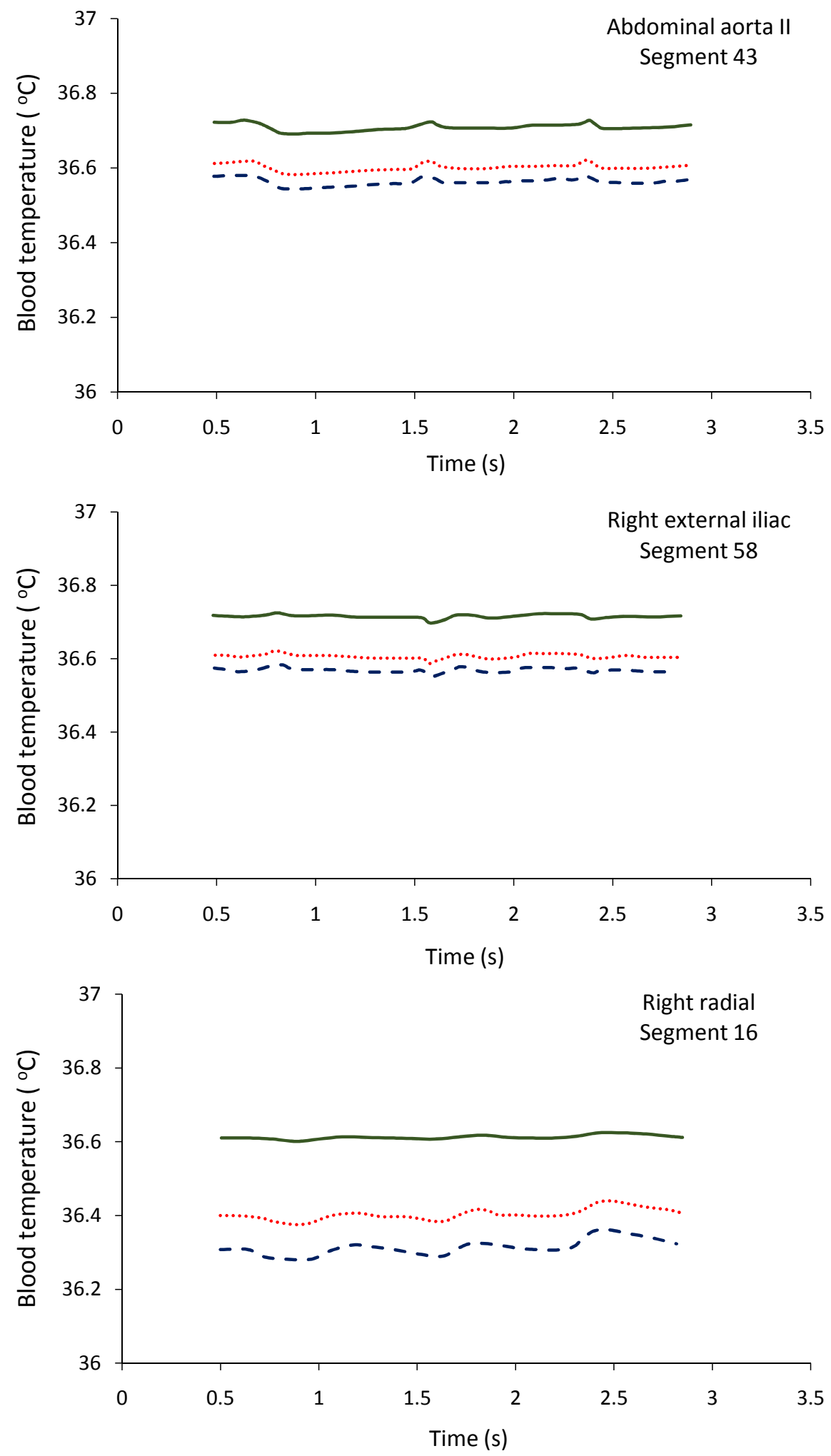

Figure 3.7: Blood temperature distributions in three locations and under various outside conditions. 

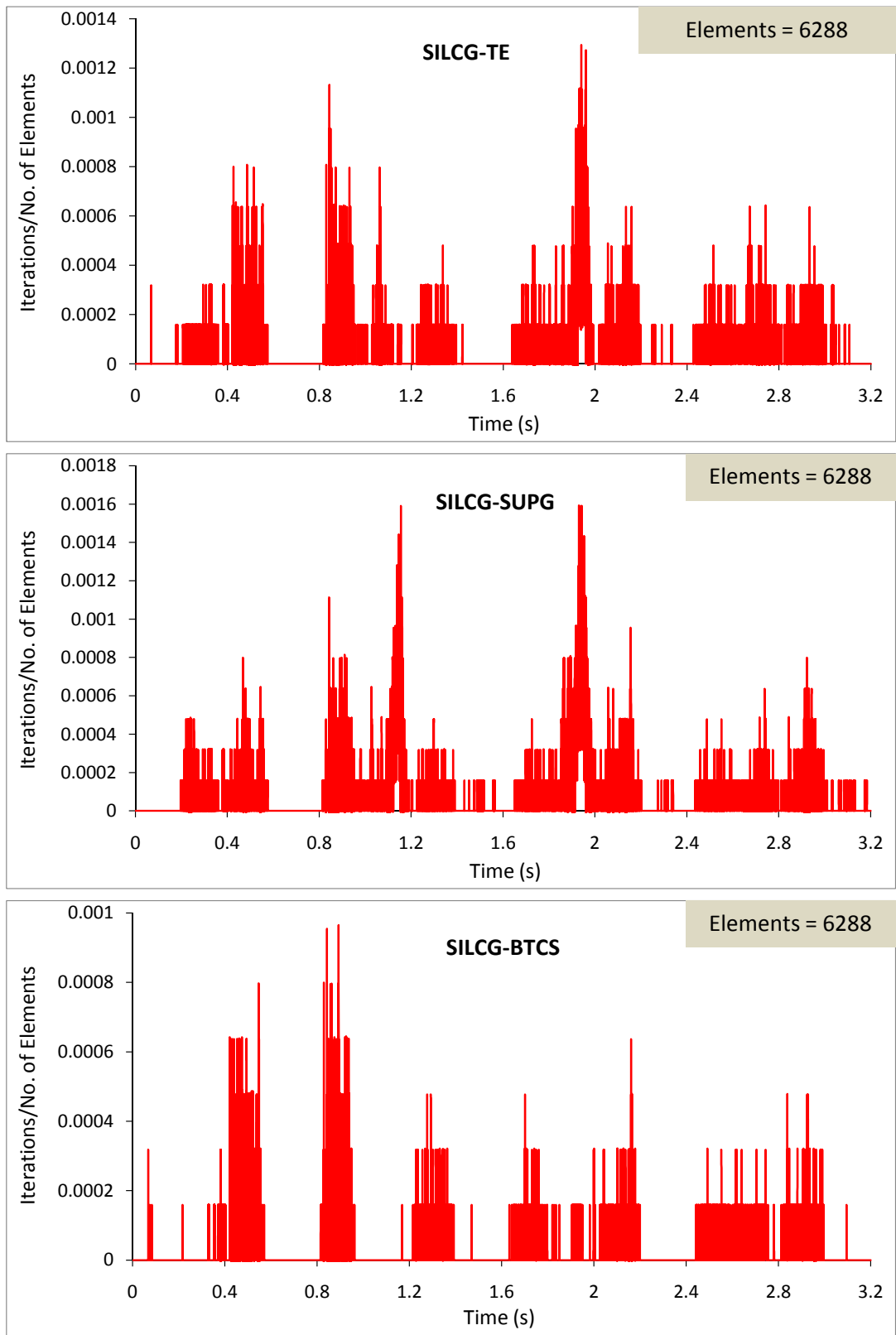

Figure 3.8: Number of iterations to number of elements ratio for for all three approaches. Jacobian matrix is calculated at every iteration, temperature loop is considered only. 

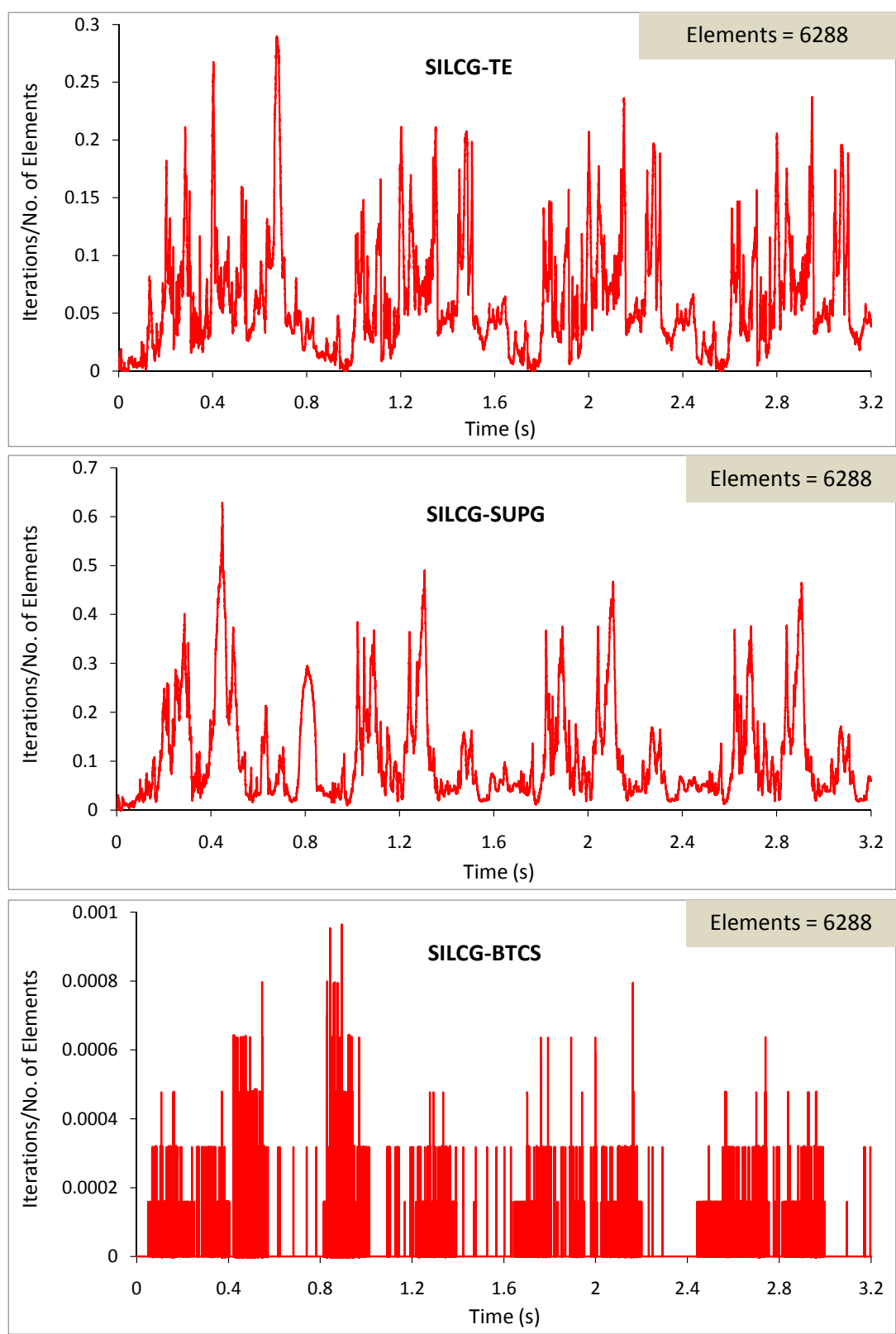

Figure 3.9: Number of iterations to number of elements ratio for for all three approaches. Jacobian matrix is calculated at every iteration, total cost is estimated as iterations/no. of elements ratio of flow+the one from the temperature loop shown in previous figure. 

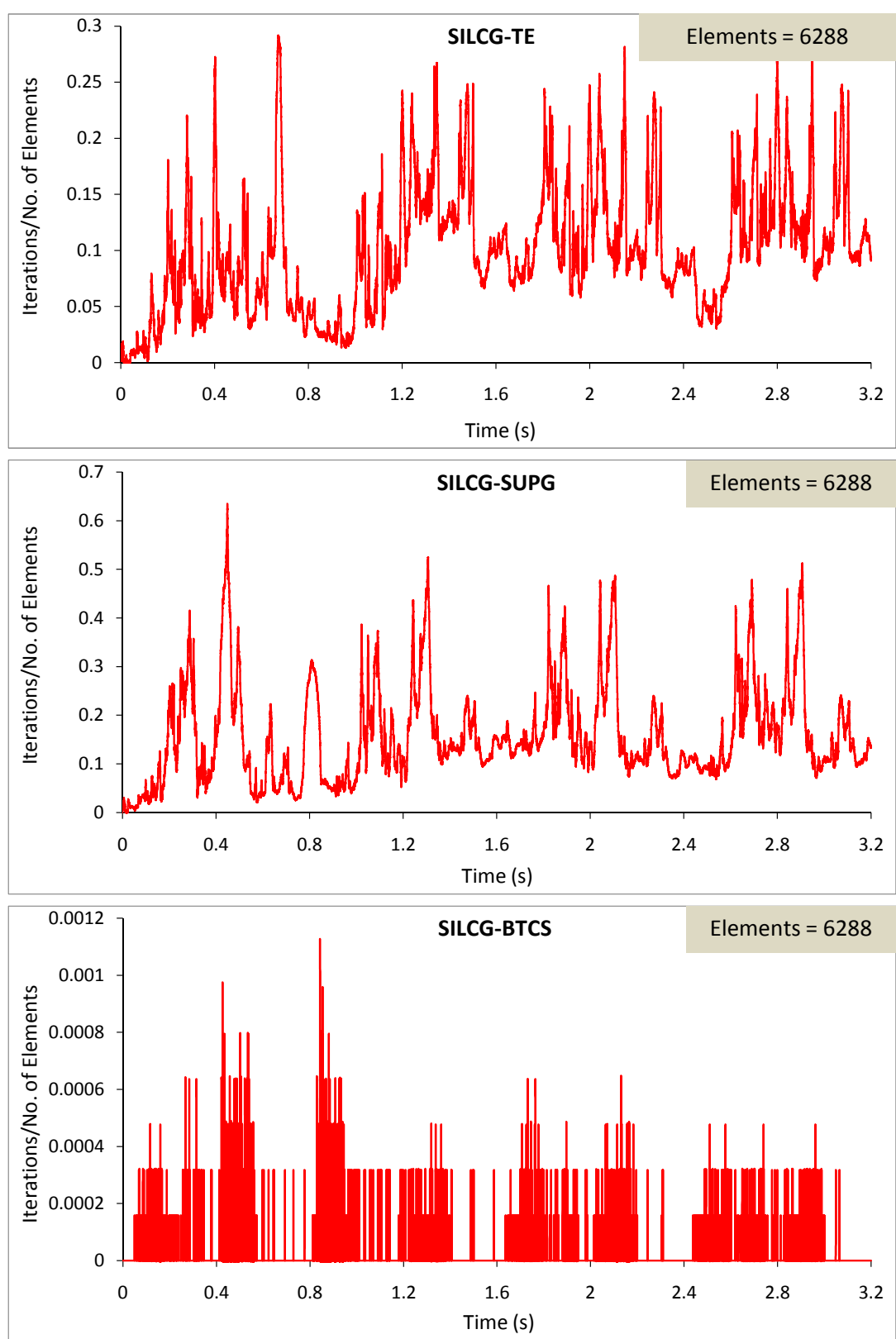

Figure 3.10: Number of iterations to number of elements ratio for for all three approaches. Jacobian matrix is calculated at every time step (not every iteration), total cost is estimated as iterations/no. of elements ratio of flow+the one from the temperature loop. 

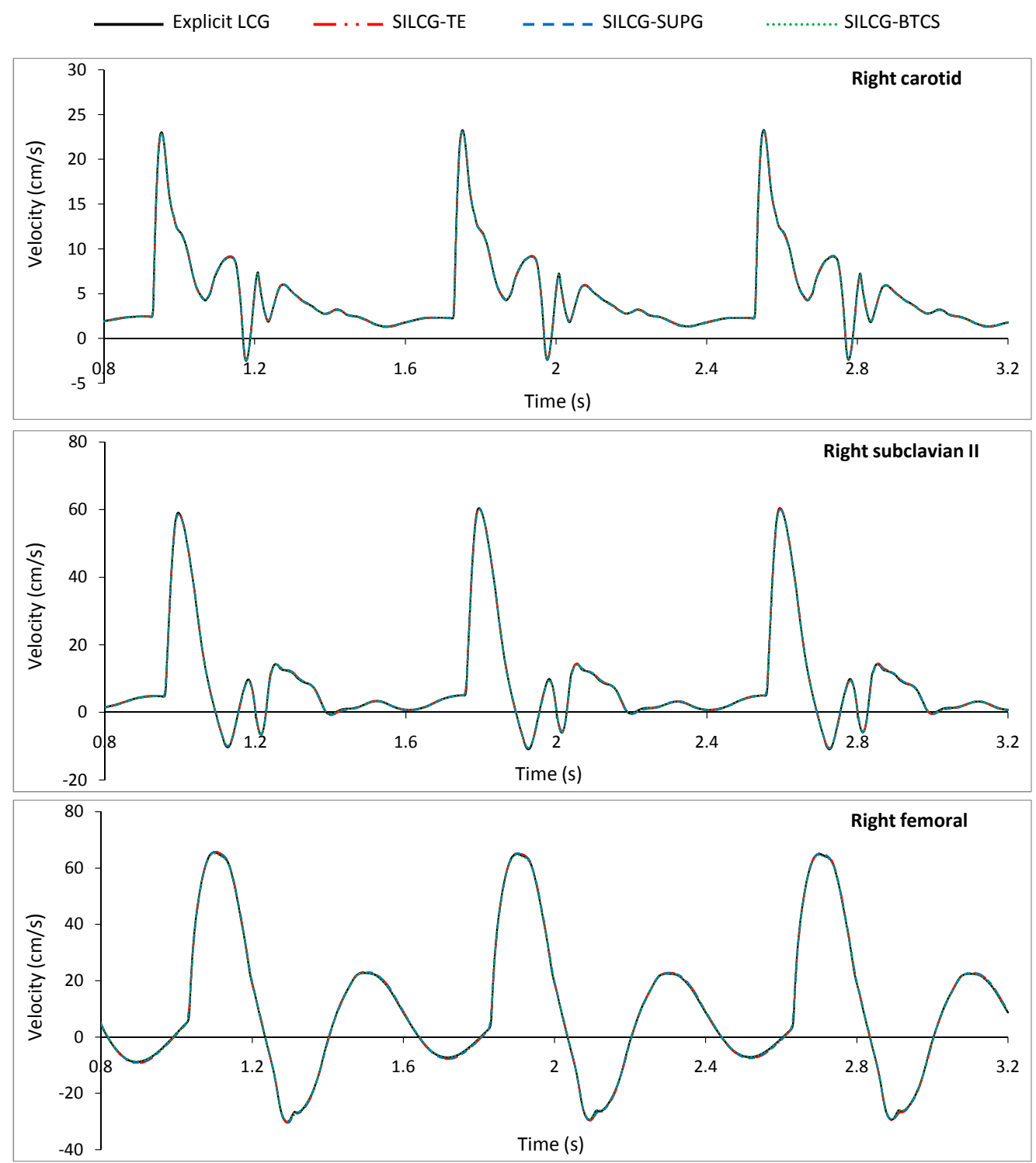

Figure 3.11: Blood velocity variation in various locations along the arterial tree. 

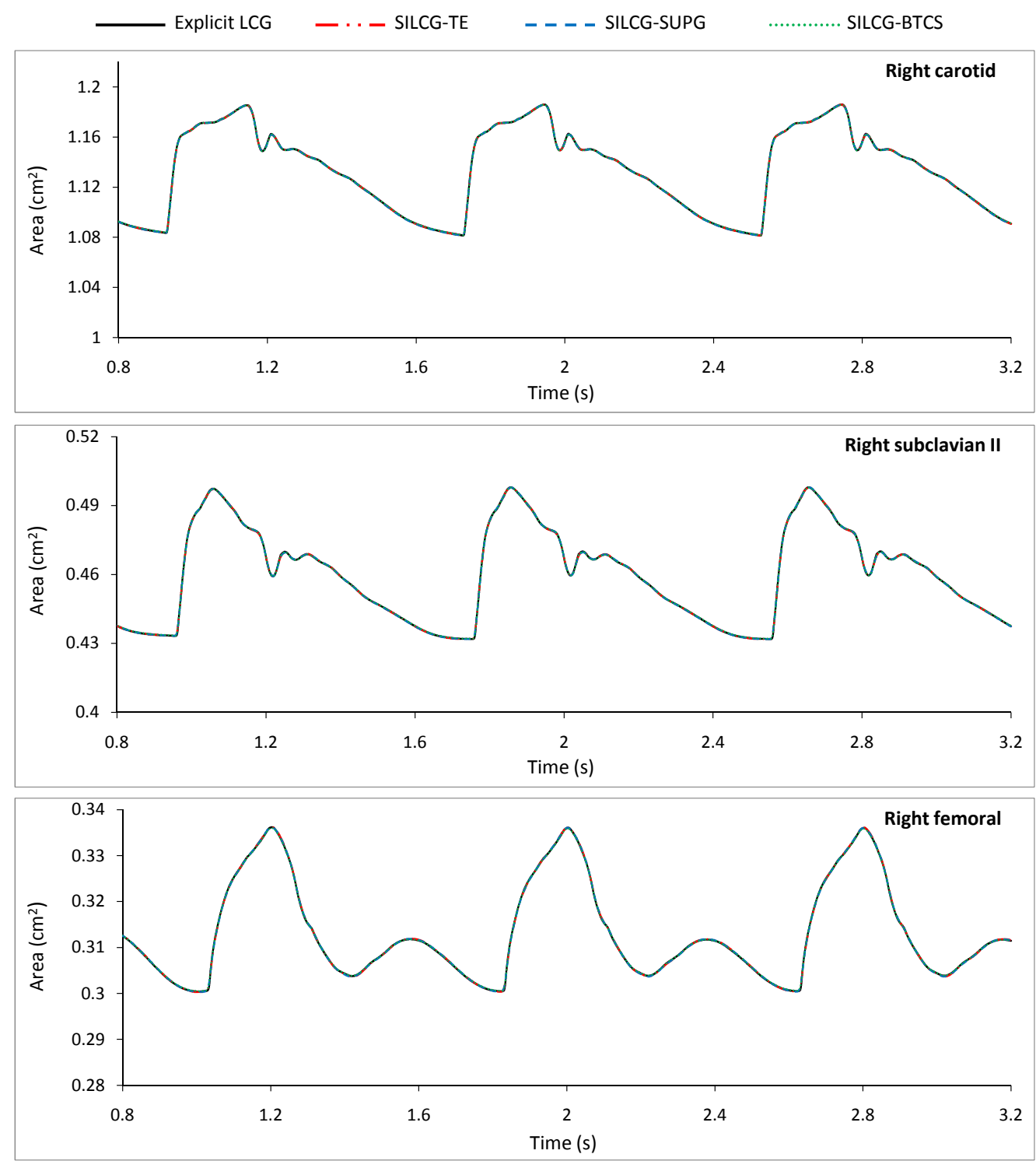

Figure 3.12: Vessel cross-section area variation in different locations along the arterial tree. 
Chapter 4

Modelling ageing effects 


\subsection{Introduction}

In this chapter, flow and thermal contribution of all major features mentioned in the previous two chapters are evaluated highlighting relevant parameters in an aged human body. In section 4.2 all the most relevant changes due to ageing are presented and then discussed by means of some numerical examples, showing the impact of such changes on the blood flow (shown by blood pressure) and thermal balance (represented by means of body thermal indicators, such as $T_{\text {core }}$ and average skin temperature $T_{\text {skin }}$ ) in section 4.3. This is then followed by a final section where conclusion remarks are derived in section 4.4.

\subsection{Modelling ageing effects on the human body}

Here an age-dependent modelling methodology describing flow and heat transfer within a human body is presented. The whole system can be subdivided into 3 major subcomponents: the arterial systemic circulation, the solid tissues, and the thermo-regulatory system. For each model sub-component, the formulations/parameters dependencies on the age $(\xi)$ are highlighted.

\subsubsection{Arterial system}

The larger arterial system is represented with a network of elastic tubes shown in Figure 2.5 , with the left ventricle as an inlet of the circuit and peripheral circulation as terminals. For an artery, the wall stiffness variations with age can be estimated from the changes of the pulse wave velocity, as proposed in $[7,1]$. The pulse wave velocity is the intrinsic wave speed $c$ given in Eq. (2.30). Rewriting same equation gives

$$
c=\sqrt{\frac{\beta \sqrt{A}}{2 \rho}} .
$$


Since $c$ doubles from age of 20 to 80 years, they decreased compliance by a factor of four for such age range. In the work of [1], a set of empirical inverse relationships between arterial diameter $(d)$ and local pulse wave velocity was derived for all age decades as

$$
c=\frac{a}{d^{b}},
$$

where $a$ and $b$ are fitting coefficients depending on the age decade (see table 4.1). As a local pulse wave velocity was estimated, the main lumen diameter is assumed unchanged with age [1]. By combining Eq. (4.1) and (4.2) it is possible calculating $\beta$ for each

Table 4.1: Age-dependent fitting coefficients of Eq.(4.2), taken from [1].

\begin{tabular}{|c|c|c|}
\hline$\xi(\mathrm{yr})$ & $a$ & $b$ \\
\hline 30 & 15.48 & 0.502 \\
40 & 15.59 & 0.458 \\
50 & 16.33 & 0.447 \\
60 & 16.68 & 0.428 \\
70 & 15.91 & 0.372 \\
80 & 15.29 & 0.345 \\
\hline
\end{tabular}

artery. The work of [66] showed that arterial distensibility decreases significantly only in coronary arteries while in femoral arteries the variation is modest. From such study, the percentage decrease in distensibility (stiffness doubled from $30 \mathrm{yr}$ to $60 \mathrm{yr}$ ) well matches the assumption made by [7]. It is noteworthy to mention that uniform stiffening augmentation should mainly be considered for arteries belonging to the body trunk and not localized in the limbs.

Fluid thermal properties such as specific heat $\left(c_{p}\right)$ and thermal diffusivity $(\alpha)$ are also considered constant. The inner wall heat transfer coefficient $h_{\text {in }}$ is calculated following [118] (Nusselt number is assumed to be equal to 4). The solutions algorithms for flow 
and heat transfer characteristics have already been developed in both previous chapters, so the ageing related aspects will be only detailed here.

\section{Arterial tree}

As detailed in the first chapter, the arterial tree shown in Figure 2.5 is composed of 63 segments (plus 28 tapering vessels for describing terminals boundaries). The geometry and structural properties of this vasculature are considered, according to the waveforms showed in [12], to be representative for a young adult body ( $\left.\xi_{0}=30 \mathrm{yr}\right)$.

The terminal part of the larger arterial network, also called microcirculation, represents the site of greatest pressure drop in the arterial blood circuit [119]. Since the number of arterial branches increases dramatically towards the periphery, it is generally convenient representing the peripheral circulation effects by imposing specific conditions at the outlet nodes of the large arterial network. For modelling such boundary conditions, several strategies can be adopted. Among these, the Windkessel model and tapering vessels represent popular choices. In the first case a vascular resistance $\left(R_{T}\right)$ and a characteristic impedance $\left(Z_{T}\right)$ define the terminal behavior, whilst in the latter, the terminals resistance can be modulated by varying the area and thus $\beta$ of the taper. In several studies, $[120,22,113]$ no phase difference between reflected flow and pressure at the terminals was assumed, and the downstream effects on flow were considered purely resistive. Terminal reflections can be described assuming that the change in the outgoing characteristic is determined from the change in the incoming characteristic [121], so Eq. (2.43) is modified to be

$$
w_{b}^{n+1}=w_{b}^{0}-R_{R}\left(w_{f}^{n+1}-w_{f}^{0}\right),
$$

where $R_{R}$ is the reflection coefficient, $w_{f}^{0}$ and $w_{b}^{0}$ are the initial values of forward and backward characteristics variables $w_{f}$ and $w_{b}$. In the current study extremities are represented by tapering vessels. These terminal vessels present a step decrease in $A_{0}$ (and 
therefore a step increase in $\beta$ ) which allows accounting for the characteristic reflections of the downstream vasculature.

The study of [67] showed that the age-related decrease in elasticity occurring for larger arteries is not fully paralleled by an increase in arterial impedance. Based on this, [7] accounted for such effect by increasing linearly $(+5 \%$ per decade) the terminal resistance $R_{T}$ from $0.8 \mathrm{mmHg} \mathrm{s} / \mathrm{ml}$ at $20 \mathrm{yr}$ to $1.04 \mathrm{mmHg} \mathrm{s} / \mathrm{ml}$ at $80 \mathrm{yr}$. This can be done also for the tapering model, by varying $\beta$ of each vessel accordingly. It is important to note that the modulation of terminal resistance may affect significantly the cardiac output (CO). Based on experimental evidence $[122,123,89]$, terminals changes are modelled in [1] by assuming that the resting cardiac output is age-independent. They assumed that the resistance increase is inline with the mean arterial pressure rise. From the above mentioned works, it is possible extrapolating (via least square method) the expressions reported in table 4.2. It is worth to say that modifications of peripheral resistance must be such that cardiac output remains within a defined range (like 6-7 L/min).

Table 4.2: Set of equations used for modelling terminals variations.

\begin{tabular}{|l|c|c|}
\hline & $\xi_{0}(\mathrm{yr})$ & Equation \\
\hline Terminal coefficient $\frac{R_{T}}{R_{T, 0}}$ & 20 & $0.7722+0.009365 \xi-6.14910^{-5} \xi^{2}[1]$ \\
Terminal coefficient $\frac{R_{T}}{R_{T, 0}}$ & 20 & $0.9+0.005 \xi[7]$ \\
\hline
\end{tabular}

The temperature condition at the inlet is straightforward to prescribe and it is associated with the core tissue temperature (see section 3.2.7). At the exiting nodes temperature is extrapolated in time if velocity is positive; otherwise, the fluid is assumed to be in thermal equilibrium with the interacting tissue node.

\section{Heart model}

The pumping action of the heart is defined by using a lumped model, which accounts for both left ventricle (LV) dynamics and aortic valve (AV) reflections. The cardiac con- 
tractile function is commonly represented by a time-varying elastance model of the left ventricle $[124,125]$. This elastance curve $\left(E_{L V}\right)$ is commonly used for linking the left ventricular pressure $p_{L V}$ to the chamber volume $V_{L V}$ :

$$
p_{L V}=E_{L V}\left[V_{L V}-V_{L V, 0}\right]
$$

where $V_{L V, 0}$ is the unloaded ventricular volume. The elastance curve varies cyclically between values defined as end-diastolic elastance $\left(E_{L V, \min }\right)$ and end-systolic elastance $\left(E_{L V, \max }\right)$. The elastance curve was approximated Stergiopulos in [126] by means of a periodic "double Hill" function given below

$$
E_{L V}=E_{L V, \min }+E_{L V, \max } \psi\left(\frac{\frac{t}{\zeta_{1} \mathrm{~T}_{\text {card }}} \eta_{1}}{1+\frac{t}{\zeta_{1} \mathrm{~T}_{\text {card }}} \eta_{1}} \frac{1}{1+\frac{t}{\zeta_{2} \mathrm{~T}_{\text {card }}} \eta_{2}}\right)
$$

where $\psi$ is a normalization parameter, $\mathrm{T}_{\text {card }}$ is the cardiac period, while $\eta_{1}, \eta_{2}, \zeta_{1}, \zeta_{2}$ are parameters determining, respectively, the steepness of the curves and the relative appearance times. The LV volume at the beginning of the cardiac cycle depends on the end-diastolic pressure $p_{E D}$ and can be calculated as $V_{L V, E D}=V_{L V, 0}+p_{E D} / E_{L V, \min }$. In the first cardiac phase (isovolumetric contraction) the chamber volume remains constant while pressure increases, untill the aortic valve opens. During the ejection phase the LV volume $V(t)$ varies in time according to [127]

$$
\frac{d V_{L V}}{d t}=-G_{A V}
$$

in which $G_{A V}$ is the net flow at the inlet node (after the AV). Once the elastance function $E_{L V}$ reaches $E_{L V, \max }$, the ejection phase ends. This is followed by the isovolumetric relaxation phase, which lasts untill $p_{L V}$ equalizes the end-diastolic pressure $\left(p_{E D}\right)$. At this point the refilling phase starts, and the volume increase can be computed as follows [126]

$$
\frac{d V_{L V}}{d t}=\frac{p_{v e n}-p_{L V}}{R_{v e n}}
$$


where $p_{v e n}$ and $R_{v e n}$ represent, respectively, the pressure and the flow resistance of the venous system (the latter is assumed to be equal to $0.0125 \mathrm{mmHg} \mathrm{s} / \mathrm{mL}$ ). It is important to note that for both Eq. (4.6) and (4.9) the solution is sought by employing forward Euler's method. It is important to mention that for modelling the left ventricular contraction models such as $[128,129]$ represent comprehensive modelling alternatives. The LV pressure $p_{L V}$ is used for evaluating the inlet forward characteristic variable $w_{f, i n}^{n+1}$, defined as

$$
w_{f, i n}^{n+1}=w_{b}^{0}+4 \sqrt{\frac{2}{\rho}} \sqrt{\left(p_{L V}-p_{e x t}\right)+\beta \sqrt{A_{0}}} .
$$

Depending on the AV state, the forward characteristic variable generated in the LV can be completely reflected, partially or completely transmitted to the arterial system. This is carried out in the model by varying periodically in time the AV transmission coefficient $\left(R_{A V}\right)$ from 0 to 1 . The valve regulation scheme is extensively illustrated in $[121,12]$ and yields

$$
w_{f}^{n+1}=w_{f}^{0}+\left(1-R_{A V}\right)\left(w_{f, i n}^{n+1}-w_{f}^{0}\right)-R_{A V}\left(w_{b}^{n+1}-w_{b}^{0}\right),
$$

in which $w_{f}^{n+1}$ can be extrapolated backwards in time, similarly to what done for the terminal elements (see Eq.(2.44)). This means the input pressure wave shown in Figure 2.2 will be age-dependent as show in Figure 4.1. The figure shows the modified input pressure wave (age-dependent-wave). This comes as a result of the use of the elastance model for the heart left ventricle. In a quick analysis for the new input pressure, it is obvious that the peak pressure is no longer constant over the ejection period as it was for the normal case shown in Figure 2.2. This refers to the higher reflections that occur in the aorta. Also, it can be seen that the input waves are moving faster with the age referring to the harder work that required from the heart to overcome the reflections and to keep blood circulated in the body.

The works of $[7,1]$ showed how to modify the cardiac function in order to account for ageing effects. Others like [7] assumed that the ventricular wall stress is preserved with advancing age and thus $E_{L V, \max }$ was increased proportionally to the systolic pressure. 


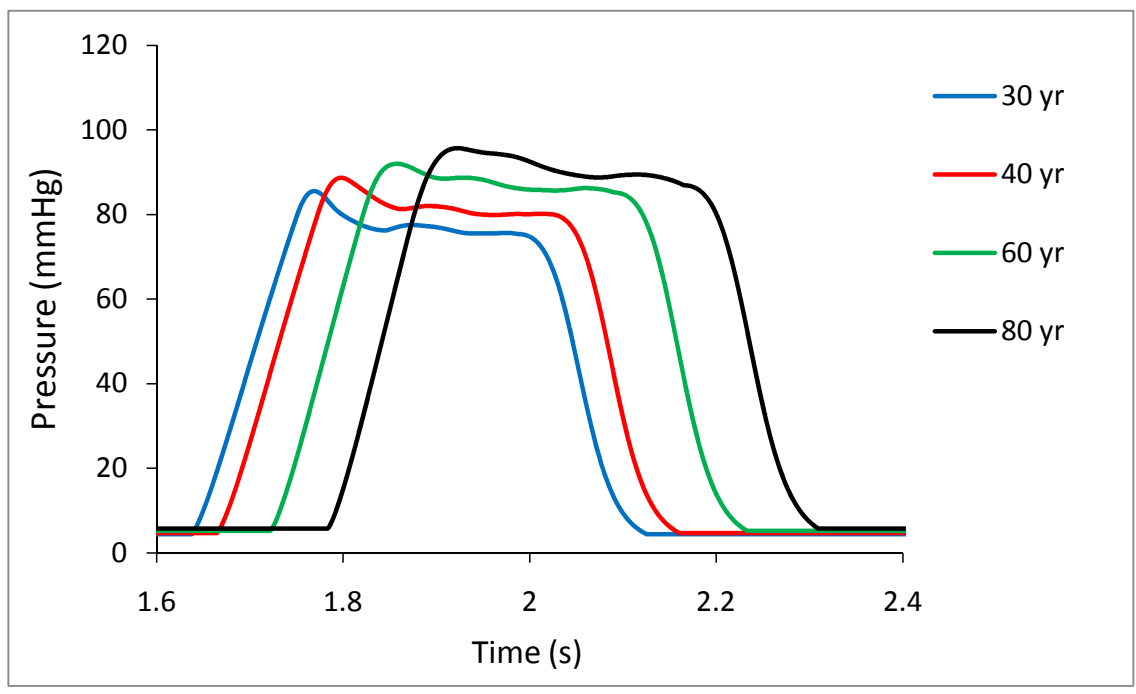

Figure 4.1: Input pressure wave for different ages.

It is important to note that the hypertrophy was related to neither diastolic strain nor ventricular wall stress. Since the LV stiffening renders ventricular filling slower, affecting the diastolic function, $E_{L V, \min }$ was augmented proportionally to $E_{L V, \max }$ increase. The enddiastolic volume $\left(V_{L V, E D}\right)$ is assumed unchanged as well during cardiac remodelling. In order to maintain a constant $V_{L V, E D}$ with advancing age, the end-diastolic pressure ( $\left.p_{E D}\right)$ was increased appropriately. According to the physiological data [130], the authors of [1] modelled heart rate (HR) age-decrease from $73.3 \mathrm{bpm}$ at $30 \mathrm{yr}$ to 67.3 at $80 \mathrm{yr}$. Table 4.3 summarizes all reference data used to model LV coefficients for each decade.

\subsubsection{Solid system}

The arterial architecture within the tissues is as same as used in the previous chapter shown in Figure 3.2. The multi cylinders approach is considered by adjusting the agerelated parameters.

It is known that also body tissues are subjected to age-induced modifications. In [74] muscle loss was recorded by considering the arm muscle circumference decrease with 
Table 4.3: $\mathrm{LV}$ reference data for each age decade. Values for $p_{E D}$ were obtained by readapting the age-relationship extrapolated from [7] to a reference value $4.5 \mathrm{mmHg}$ at $35 \mathrm{yr}$.

\begin{tabular}{|c|c|c|c|c|}
\hline$\xi(\mathrm{yr})$ & $E_{L V, \max }(\mathrm{mmHg} / \mathrm{mL})[7]$ & $E_{L V, \min }(\mathrm{mmHg} / \mathrm{mL})[7]$ & $p_{E D}(\mathrm{mmHg})$ & $\mathrm{HR}(\mathrm{bpm})[1]$ \\
\hline 20 & 1.00 & 0.02500 & 4.134 & 73.3 \\
30 & 1.03 & 0.02575 & 4.410 & 73.3 \\
40 & 1.09 & 0.02725 & 4.687 & 72.1 \\
50 & 1.16 & 0.02750 & 4.964 & 70.9 \\
60 & 1.24 & 0.03100 & 5.240 & 69.7 \\
70 & 1.35 & 0.03375 & 5.517 & 68.5 \\
80 & 1.51 & 0.03755 & 5.794 & 67.3 \\
\hline
\end{tabular}

age. This can be used for approximating the age-dependency of the arm muscle thickness $\left(h_{m u, a r m}\right)$. It is also reasonable assuming that the volume reduction rates for all other skeletal muscles, such as the ones in shoulders, forearms, thighs, and legs, are the same. Others like [131] reported, for different age groups, measurements on the fat thickness in different body regions. From such data, the average layer thickness $\left(\bar{h}_{f a t}\right)$ can be linearly described as a function of age. Also, the authors of [88] applied a reduction ratio between the fat thickness of young and old adults equal to 0.8. This is inline with what reported in [131], since the calculated $h_{f a t, a b d}$ ratio between a 20 and 80 years old is $\sim 0.82$. In the abdomen region, contrary to the limbs, fat tends to accumulate and thus the relationship extrapolated from [131] is not any more valid for such region. In the study of [132], the values of abdominal subcutaneous fat and muscle thickness were reported for different age groups $(24.2 \pm 3.55-72.8 \pm 1.91 \mathrm{yr})$. With regard to the fat, the thickness increases with age from $0.151 \mathrm{~cm}$ up to $0.19 \mathrm{~cm}$, whilst the muscle tissue thickness decreases from $0.139 \mathrm{~cm}$ for young, up to $0.098 \mathrm{~cm}$ for elderly. The skin is subjected, with age, to functional and structural changes, which lead to a thinning of the layer. In [131] the average cutaneous thickness $\left(\bar{h}_{s k}\right)$ measured for different ages is also reported. 
For a more realistic description of the bone density age-reduction, the set of measurements on male trochanter bone density reported in [133] can be used. Changes in density affects also properties of the tissue, such as thermal conductivity. Walker et al. [134] extrapolated a biquadratic relationship between bone thermal conductivity $\left(k_{t, b o}\right)$ and density:

$$
\left.k_{t, b o}=0.0343+0.9935 \rho_{b o}-0.5305 \rho_{b o}{ }^{2}(W /(m K))\right) .
$$

Novieto [81] reported a weight measurements dataset for different age groups [135], from which it is possible calculate the body weight $(B W)$ as a function of age

$$
B W=51.64+1.328 \xi-0.01384 \xi^{2}(k g)
$$

where $\xi$ refers to the age. From [80] an regression curve describing resting metabolic rate $R M R$ with age can be extrapolated

$$
R M R=1.134+0.008 \xi-0.00013 \xi^{2}(\mathrm{kcal} / \mathrm{min})
$$

The body metabolic volumetric production $\overline{q_{v}}$ can be estimated by dividing Eq. (4.12) by Eq. (4.11)

$$
\overline{q_{v}}=\frac{0.06978 \bar{\rho}\left(1.134+0.008 \xi-0.00013 \xi^{2}\right)}{51.64+1.328 \xi-0.01384 \xi^{2}}\left(W / \mathrm{cm}^{3}\right)
$$

in which $\bar{\rho}$ is the average density of the body (which can be assumed constant equal to $1.17 \mathrm{~g} / \mathrm{cm}^{3}$, from [136]). It is acceptable assuming that for each body tissue the metabolic production variation with age is the same $\frac{\partial\left(q_{v} / q_{v 0}\right)}{\partial \xi} \approx \frac{\partial\left(\bar{q}_{v} / \bar{q}_{v 0}\right)}{\partial \xi}$, in which $q_{v 0}$ and $\bar{q}_{v 0}$ are volumetric rates for young adults $\left(\xi_{0}=20 \mathrm{yr}\right)$. Therefore, the tissue metabolic volumetric heat generation may calculated for each age as

$$
\frac{q_{v}}{q_{v, 0}}=1.152-0.008897 \xi+5.59610^{-5} \xi^{2} .
$$

Table 4.4 summarises the set of equations employed for modelling tissue variations. 
Table 4.4: Set of extrapolated equations used for modelling tissue variations.

\begin{tabular}{|c|c|c|c|}
\hline & Body segment & $\xi_{0}(\mathrm{yr})$ & Equation \\
\hline Muscle thickness $\frac{h_{m u, a r m}}{h_{m, a r m, 0}}$ & arm, forearm & 20 & $1.070122-0.003506 \xi[74]$ \\
Muscle thickness $\frac{h_{m u, a b d}}{h_{m u, a b d, 0}}$ & abdomen & 24.2 & $1.14687-0.00607 \xi[132]$ \\
Fat thickness $\frac{\bar{h}_{\text {a }}}{\bar{h}_{f a t}, 0}$ & any except abdomen & 20 & $1.05938-0.002969 \xi[131]$ \\
Fat thickness $\frac{h_{\text {fat, abd }}}{h_{f a t, a b d, 0}}$ & abdomen & 24.2 & $0.87139-0.005314 \xi[132]$ \\
Skin thickness $\frac{\bar{h}_{s k}}{h_{s k, 0}}$ & any & 20 & $1.01026-0.000513 \xi[131]$ \\
Bone density $\frac{\bar{\rho}_{b o}}{\bar{\rho}_{b o, 0}}$ & any & 20 & $0.95086+0.00443 \xi-6.62010^{-5} \xi^{2}[133]$ \\
Metabolic volumetric rate $\frac{q_{v}}{q_{v, 0}}$ & any & 20 & $1.152-0.008897 \xi+5.59610^{-5} \xi^{2}[81]$ \\
\hline
\end{tabular}

\subsubsection{Regulatory system}

Every time the body core temperature $\left(T_{\text {core }}\right)$ and averaged skin temperature $\left(T_{\text {skin }}\right)$ differ from their thermoneutrality values, specific regulatory mechanisms intervene in order to counterbalance the energy impairment. Such thermoregulatory system is based on a network of thermoreceptors located in several parts of the body and communicating with the hypothalamus, which plays a role of controller. Again, the regulatory mechanisms considered are sweating, cutaneous vasodilation/constriction and shivering.

\section{Sweating}

The sweating ratio $\dot{m}_{\text {swe }}$ for each body segment may also be evaluated by using the formulation proposed by [56] and reformulated in [4], which accounts for ageing effects:

$$
\begin{aligned}
\dot{m}_{\text {swe }} & =\dot{m}_{\text {swe }, 0}+\chi \theta_{\text {skin }}\left\{\left[0.8 \tanh \left(0.59 \Delta T_{\text {skin }}-0.19\right)+1.2\right] \Delta T_{\text {skin }}^{*}\right. \\
& \left.+\left[5.7 \tanh \left(1.98 \Delta T_{\text {core }}-1.03\right)+6.3\right] \Delta T_{\text {core }}^{*}\right\}
\end{aligned}
$$

where $\dot{m}_{s w e, 0}$ is the unsensible water loss, $\theta_{\text {skin }}$ and $\chi$ depend on the body region

$$
\theta_{\text {skin }}=\alpha_{\text {swe }} 2^{\frac{T_{\text {skin }}-T_{\text {skin }, 0}}{10}},
$$


in which $\alpha_{\text {swe }}$ is a skin distribution coefficient associated to each body segment (see table 4.5), whilst

$$
\begin{gathered}
\Delta T_{\text {skin }}=T_{\text {skin }}-T_{\text {skin }, 0}, \\
\Delta T_{\text {core }}=T_{\text {core }}-T_{\text {core }, 0} .
\end{gathered}
$$

Table 4.5: Body parts sweating coefficients. Such parameters are obtained by re-adapating the values from [4] for the current body solid architecture. The coefficients for the arm/forearm and the thig/leg are obtained dividing respectively, the sum of the coefficients for arms and hands, and the sum of coefficients for legs and feet by 4 .

\begin{tabular}{|c|c|c|c|c|c|c|c|}
\hline Parameter & Head (and face) & Neck & Thorax & Abdomen & Shoulders & Arm/forearm & Thigh/leg \\
\hline$\alpha_{\text {swe }}$ & 0.149 & 0.042 & 0.101 & 0.181 & 0.0185 & 0.0455 & 0.077 \\
$\chi$ & 1.0 & 1.0 & 1.0 & 1.0 & 1.0 & 1.0 & 0.6 \\
\hline
\end{tabular}

The temperature thresholds $\Delta T_{\text {skin }}^{*}$ and $\Delta T_{\text {core }}^{*}$ are defined as

$$
\begin{aligned}
& \Delta T_{\text {skin }}^{*}=\left\{\begin{array}{l}
0 \text { if } \Delta T_{\text {skin }}<\Delta T_{\text {skin,dec }} \\
\Delta T_{\text {skin }}-T_{\text {skin,dec }} \text { if } \Delta T_{\text {skin }}>\Delta T_{\text {skin, dec }}
\end{array}\right. \\
& \Delta T_{\text {core }}^{*}=\left\{\begin{array}{l}
0 \text { if } \Delta T_{\text {core }}<\Delta T_{\text {core }, \text { dec }} \\
\Delta T_{\text {core }}-\Delta T_{\text {core }, \text { dec }} \text { if } \Delta T_{\text {core }}>\Delta T_{\text {core }, \text { dec }}
\end{array}\right.
\end{aligned}
$$

where $\Delta T_{\text {skin,dec }}$ and $\Delta T_{\text {core,dec }}$ are thresholds taking into account the decline of the thermal sensitivity associated to the thermoregulatory signal (see table 4.6).

In [4] $\dot{Q}_{\text {swe }}$ the skin evaporative losses are then evaluated via

$$
\dot{Q}_{\text {swe }}=\min \left(\frac{40.6 \dot{m}_{\text {swe }}}{A_{\text {skin, }, \text { lob }}}, 2.2 h_{\text {swe }} f_{c l}\left(p_{v, s k i n}-p_{v, a}\right)\right),
$$

in which $A_{s k i n, g l o b}$ is the global body skin surface, $p_{v, s k i n}$ is the water vapour pressure at the skin (normally assumed to be that of saturated water vapour at skin temperature), $p_{v, a}$ is the water pressure in the air, $f_{c l}$ is the clothing area factor (the surface of the clothed body 
Table 4.6: Decline of the thermal sensitivity $\Delta T_{\text {skin,dec }}$ and $\Delta T_{\text {core,dec }}$ with age, from [4].

\begin{tabular}{|c|c|c|}
\hline$\xi(\mathrm{yr})$ & $\Delta T_{\text {skin,dec }}\left({ }^{\circ} \mathrm{C}\right)$ & $\Delta T_{\text {core,dec }}\left({ }^{\circ} \mathrm{C}\right)$ \\
\hline$<50$ & 0.0 & 0.0 \\
$>50$ and $<65$ & 1.5 & 0.6 \\
$>65$ and $<70$ & 1.5 & 0.6 \\
$>70$ & 1.5 & 0.4 \\
\hline
\end{tabular}

divided by the area of the nude body), and $h_{\text {swe }}$ is the evaporative heat transfer coefficient, which can be calculated as in [137].

\section{Cutaneous vasodilation/vasoconstriction}

During hot exposure, elderly people present a reduced cutaneous blood perfusion with respect to young adults [138]. The body temperature threshold for triggering such mechanism may be assumed to be lower as well in aged adults. Similarly, with advancing age, the blood perfusion to the skin during cold condition does not decrease as much as it does for a young adult. According to to [139], the cutaneous blood flow attenuation for aged people (60-90 yr) is in the order of $25-50 \%$ with respect to one of the young adults. In the study of [4] such variations do not lead to a significant difference in the global body thermal response. Similarly, [93] proposed an model accounting for such age-related vasocutaneous flow perfusion variations. In this work, a reduction of the lower threshold for maximum vasoconstriction (assumed to be $10.2^{\circ} \mathrm{C}$ ) led to a better match with the measurements obtained on older adults. Based on experimental evidence [140], the elderly threshold for maximum constriction was lowered by $0.5^{\circ} \mathrm{C}$ representing the delay of the thermal signal transmission, whilst the dilatation threshold, which is controlled by $T_{\text {core }}$ was increased by $0.05{ }^{\circ} \mathrm{C}$ over the reference value used for younger people. For an aged body ( $>50 \mathrm{yr}$ ), the following cases are considered [6] 


$$
\begin{gathered}
\dot{m}_{\text {skin,dil }}=\left\{\begin{array}{l}
\dot{m}_{\text {skin }, 0} \text { if } T_{\text {core }} \leq 36.85{ }^{\circ} \mathrm{C}, \\
\frac{T_{\text {core }}-36.85}{37.25-36.85}\left(\dot{m}_{\text {skin, } \text { max }}-\dot{m}_{\text {skin }, 0}\right)+\dot{m}_{\text {skin }, 0}(\mathrm{~kg} / \mathrm{s}) \\
\quad \text { if } 36.85{ }^{\circ} \mathrm{C}<T_{\text {core }}<37.25{ }^{\circ} \mathrm{C}, \\
\dot{m}_{\text {skin,max }} \text { if } T_{\text {core }} \geq 37.25{ }^{\circ} \mathrm{C},
\end{array}\right. \\
\dot{m}_{\text {skin,con }}=\left\{\begin{array}{l}
\dot{m}_{\text {skin,min }} \text { if } T_{\text {skin }} \leq 10.2{ }^{\circ} \mathrm{C}, \\
\frac{T_{\text {skin }}-10.2}{33.2-10.2}\left(\dot{m}_{\text {skin }, 0}-\dot{m}_{\text {skin,min }}\right)+\dot{m}_{\text {skin, }, \text { min }}(\mathrm{kg} / \mathrm{s}) \\
\dot{m}_{\text {skin,min }} \text { if } T_{\text {skin }} \geq 33.2{ }^{\circ} \mathrm{C},
\end{array}\right.
\end{gathered}
$$

where $\dot{m}_{s k, 0}, \dot{m}_{s k, d i l}$ and $\dot{m}_{s k, c o n}$ are respectively the basal, the dilation and the constriction flow rates. The basal skin flow $\dot{m}_{\text {skin, } 0}$ can be considered age-independendent [93], whilst for the maximum and minimum cutaneous flows the following formulations, derived by linearly interpolation of data from [139], are used

$$
\begin{gathered}
\dot{m}_{\text {skin,min }}=\frac{\dot{m}_{\text {skin, } \min , 0}}{(1.198-0.007677 \xi)} . \\
\dot{m}_{\text {skin,max }}=\dot{m}_{\text {skin,max }, 0}(1.198-0.007677 \xi) .
\end{gathered}
$$

in which $\dot{m}_{s k i n, \min , 0}$ and $\dot{m}_{s k i n, \max , 0}$ are the corresponding values for an young adult of 20 yr old. $\dot{m}_{s k i n, 0}, \dot{m}_{s k i n, \max }$ and $\dot{m}_{s k i n, \min }$ are reported for each segment in table 4.7.

\section{Shivering}

Such mechanism occurs only when $T_{\text {core }}$ is equal or lower than $37.1{ }^{\circ} \mathrm{C}$. According to to [84] the shivering threshold $\left(T_{\text {shiv }}\right)$ is a function of the core temperature 
Table 4.7: Body parts cutaneous perfusion coefficients. Such parameters are obtained by readapating the values from [8] for the current body solid architecture. It is important to note that the coefficients for the neck and shoulder are assumed to be equal respectively to the coefficents for the head and thorax.

\begin{tabular}{|c|c|c|c|}
\hline Body segment & $\dot{m}_{\text {skin, },}\left(\mathrm{cm}^{3} / \mathrm{s}\right)$ & $\dot{m}_{\text {skin, } \max }\left(\mathrm{cm}^{3} / \mathrm{s}\right)$ & $\dot{m}_{\text {skin, } \min }\left(\mathrm{cm}^{3} / \mathrm{s}\right)$ \\
\hline Head/neck & 1.681 & 1.255 & 4.598 \\
Thorax (chest)/shoulder & 0.956 & 0.0 & 9.235 \\
Abdomen (pelvis) & 0.631 & 0.0 & 6.098 \\
Arm & 0.253 & 0.0 & 2.311 \\
Forearm & 0.141 & 0.0 & 1.543 \\
Thigh & 0.404 & 0.0 & 3.459 \\
Leg (calf) & 0.181 & 0.0 & 2.293 \\
\hline
\end{tabular}

$$
T_{\text {shiv }}=\left\{\begin{array}{l}
35.5{ }^{\circ} \mathrm{C} \text { if } T_{\text {core }} \leq 35.8{ }^{\circ} \mathrm{C}, \\
-10222+570.9 T_{\text {core }}-7.9455 T_{\text {core }}^{2}\left({ }^{\circ} \mathrm{C}\right) \text { if } 35.8{ }^{\circ} \mathrm{C} \leq T_{\text {core }} \leq 37.1{ }^{\circ} \mathrm{C} .
\end{array}\right.
$$

Considering the whole body, the maximum metabolic heat production by shivering $\left(Q_{s h i v, \max }\right)$ is calculated as

$$
Q_{\text {shiv, } \max }=\frac{1}{3600}\left(-1.186110^{9}+6.55210^{7} T_{\text {core }}-9.041810^{5} T_{\text {core }}^{2}\right)(W) .
$$

The shivering metabolic heat generation $Q_{\text {shiv }}$ depends on the skin temperature and shivering threshold temperature

$$
Q_{\text {shiv }}=\left\{\begin{array}{l}
0 \text { if } T_{\text {skin }} \leq\left(40-T_{\text {shiv }}\right), \\
Q_{\text {shiv,max }}\left[1-\left(\frac{T_{\text {skin }}-20}{T_{\text {shiv }}-20}\right)^{2}\right](W) \text { if }\left(40-T_{\text {shiv }}\right) \leq T_{\text {skin }} \leq T_{\text {shiv }} .
\end{array}\right.
$$


The shivering volumetric source $q_{\text {shiv }}$ for a single cylinder muscle tissue is calculated as

$$
q_{\text {shiv }}=\alpha_{\text {shiv }} \frac{Q_{\text {shiv }}}{V_{\text {mus }, \text { glob }}}\left(W / \mathrm{cm}^{3}\right) .
$$

where $V_{\text {mus,glob }}$ is the global muscle volume of the body and $\alpha_{\text {shiv }}$ is a coefficient taking into account the muscle volume distribution of each body segment with respect to the total (see table 4.8).

The shivering production is less in aged people because the shivering threshold core temperature and muscle mass are lower with respect to young adults. In [141] the shivering threshold of the patients are plotted against the age. For 15 adults younger than 80 years old (58 $\pm 10 \mathrm{yr}$ ) the mean of the recorded shivering thresholds was $36.1{ }^{\circ} \mathrm{C}$, whilst older people ( 8 people, $89 \pm 7 \mathrm{yr}$ ) shivered at a significantly lower temperature $(35.2 \pm 0.8$ ${ }^{o} \mathrm{C}$ ). The reduction rate obtained by linearly interpolating such values is equal to -0.031 ${ }^{o} \mathrm{C} / \mathrm{yr}$.

Table 4.8: Body parts sweating coefficients. These parameters are obtained by re-adapting the values from [9] for the current body solid architecture. Head and shoulders include, respectively, the face and the back.

\begin{tabular}{|l|c|c|c|c|c|c|c|c|}
\hline & Head & Neck & Thorax & Abdomen & Shoulder & Arm/forearm & Thigh & Leg \\
\hline$\alpha_{\text {shiv }}$ & 0.01 & 0.01 & 0.257 & 0.364 & 0.117 & 0.0125 & 0.022 & 0.016 \\
\hline
\end{tabular}

\subsection{Results and discussion}

In this section, some simulated results are shown in order to highlight the impact of the modelling assumptions previously reported. The numerical calculations are carried out by using a framework based on the flow and bioheat transfer models developed in the previous chapter, i.e. by using SILCG-TE. The discussion will be divided into two categories 
related to flow and temperature respectively. Time step $\Delta t=0.1 \mathrm{~ms}$ and $l_{e}=0.25 \mathrm{~cm}$ for all cases.

\subsubsection{Human circulation results}

Here the age-modifications effects on flow presented in section 4.2.1 are showed and discussed. Four age groups are considered, 30, 40, 60 and 80 years old. Blood pressure is monitored at two sites of the arterial tree, ascending aorta and brachial artery (i.e. segments 9 and 15 of the network shown in Figure 2.5). The LV parameters vary with age according to table 4.3. Figure 4.2 shows how the computed elastance curve varies with age. This behavior impacts the input pressure wave as illustrated in Figure 4.1.

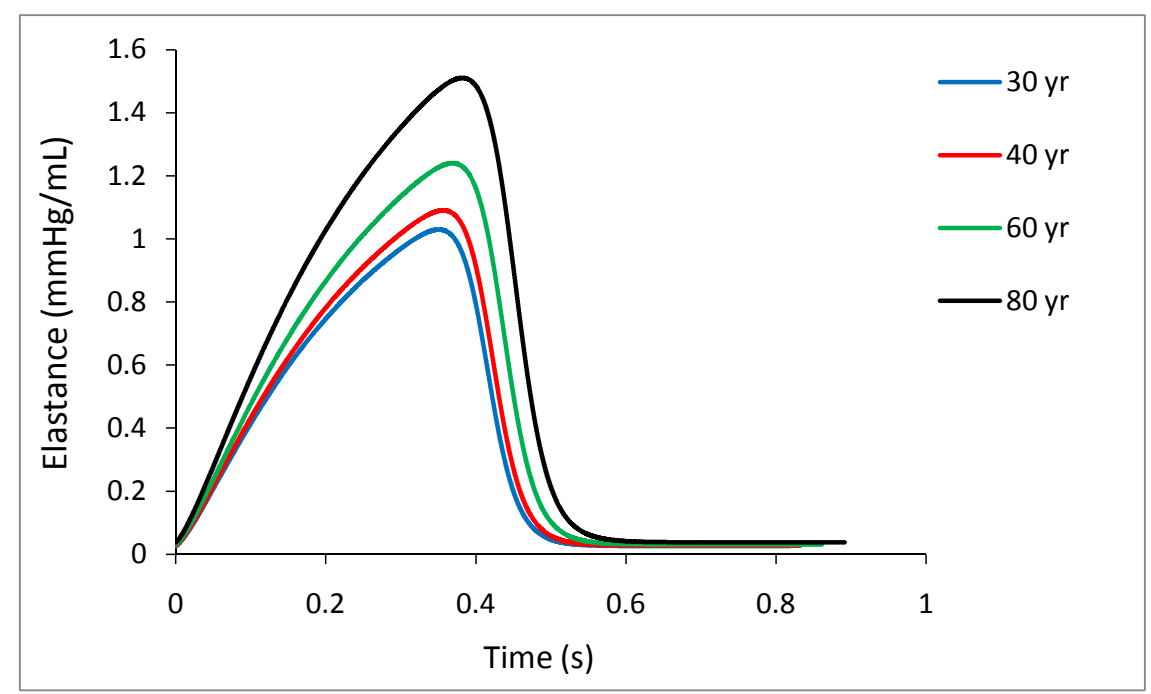

Figure 4.2: Elastance curve for different ages.

The compliance against age-variations in arteries are modelled by following the two approaches proposed: 'specific' $\beta$ augmentation accounting for pulse wave velocity (through Eq. (4.2)). So, the pulse wave velocity is estimated through Eq. (4.2) using available lumen diameter for each artery. Then, the age related $\beta$ can be calculated by rearranging Eq. (4.1). The second appoach is 'uniform' $\beta$ augmentation and it is carried out according to 
the compliance decrease reported in [7] (i.e. $\beta$ doubles from 20-80 yr), so an approperiate increment is added over every decade, and it is applied to i) all arteries and ii) only large arteries belonging to the trunk (segments 9, 10, 13, 22, 23, 26, 35, 36, 43, 45, 47 and 49). Terminal resistances ( $\beta$ of tapering vessels) are uniformly modulated in order to reflect the terminal resistance increase proposed in [7]. Fluid properties employed in the simulations as same as reported before, i.e. $\rho=1.06 \mathrm{~g} / \mathrm{cm}^{3}, \mu=3.510^{-2}$ poise, $k=0.05 \mathrm{~W} /(\mathrm{cm}$ $\left.{ }^{o} \mathrm{C}\right), c_{p}=3.9 \mathrm{~J} /\left(\mathrm{g}{ }^{o} \mathrm{C}\right)$ and $h_{i n, c o n}=0.1 \mathrm{~W} /\left({ }^{o} \mathrm{C} \mathrm{cm}^{2}\right)$.

The analysis of pressure waveforms along the arterial network is of fundamental importance for evaluating the interaction between the cardiac inflow and the vascular resistance. In the Figures 4.3 and 4.4, pressure waveforms at ascending aorta and brachial artery are reported for different ages. The figure shows that the both $\beta$ augmentation strategies lead to similar waveforms. In all cases presented, pressure amplitude is augmented with age, as well as the cardiac period. The numerical results show that specific $\beta$ augmentation approch is the closest to the ones from the reference work made by [1].

Figure 4.5 shows the variations of systolic and diastolic pressure in brachial and ascending aorta arteries against measurements. Also, in such case, the simulation results are in good agreement with the experimental observations. However, it is obvious that that limiting the age-modulation to only trunk arteries and specific one lead to better results in terms of diastolic pressure. For systolic pressure, it is again, specific $\beta$ strategy is better.

The effects of age-modelling assumptions are also evaluated by using the ratio between brachial and aortic pulse pressure (PP) as shown in Figure 4.6. The employment of such indicators allows characterizing the waveform shape. In this case, the specific $\beta$ approach seems to give less accurate results. This may be because of Eq. (4.2) only provides an accurate estimation of pulse wave velocity in large arteries, and not for all network vessels. 


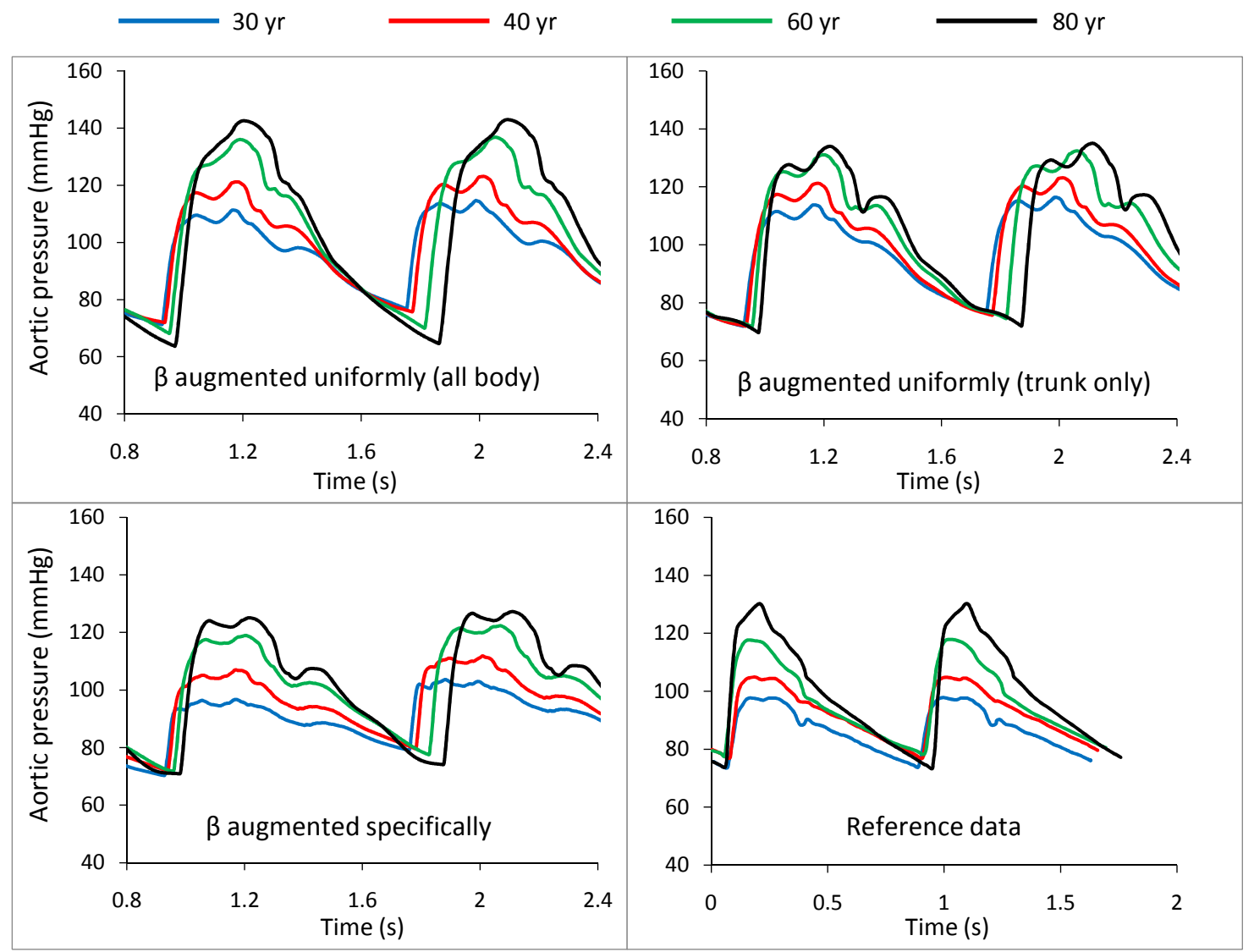

Figure 4.3: Pressure waveforms at ascending aorta artery for different ages. Reference data are taken from [1].

\subsubsection{Tissues heat transfer results}

In this section the effects on thermal balance of age modifications introduced in sections 4.2.2 and 4.2.3 are reported. For the flow system variations in the vessel, compliance is dealt with by adopting the 'specific' $\beta$ augmentation approach. The adopted body-tissues geometry corresponding to young age $\left(\xi_{0}=30 \mathrm{yr}\right)$ is defined in Table 3.1). For simulating ageing effects on the tissues the set of equations reported in Table 4.4 is employed. The methodologies describing regulatory mechanisms are modified according to section 4.2.3. In this case, a bare body is exposed to the hot environment $\left(40^{\circ} \mathrm{C}, 42 \%\right.$ r.h.) for one hour. The body is assumed to be at resting conditions (no physical activity) and 


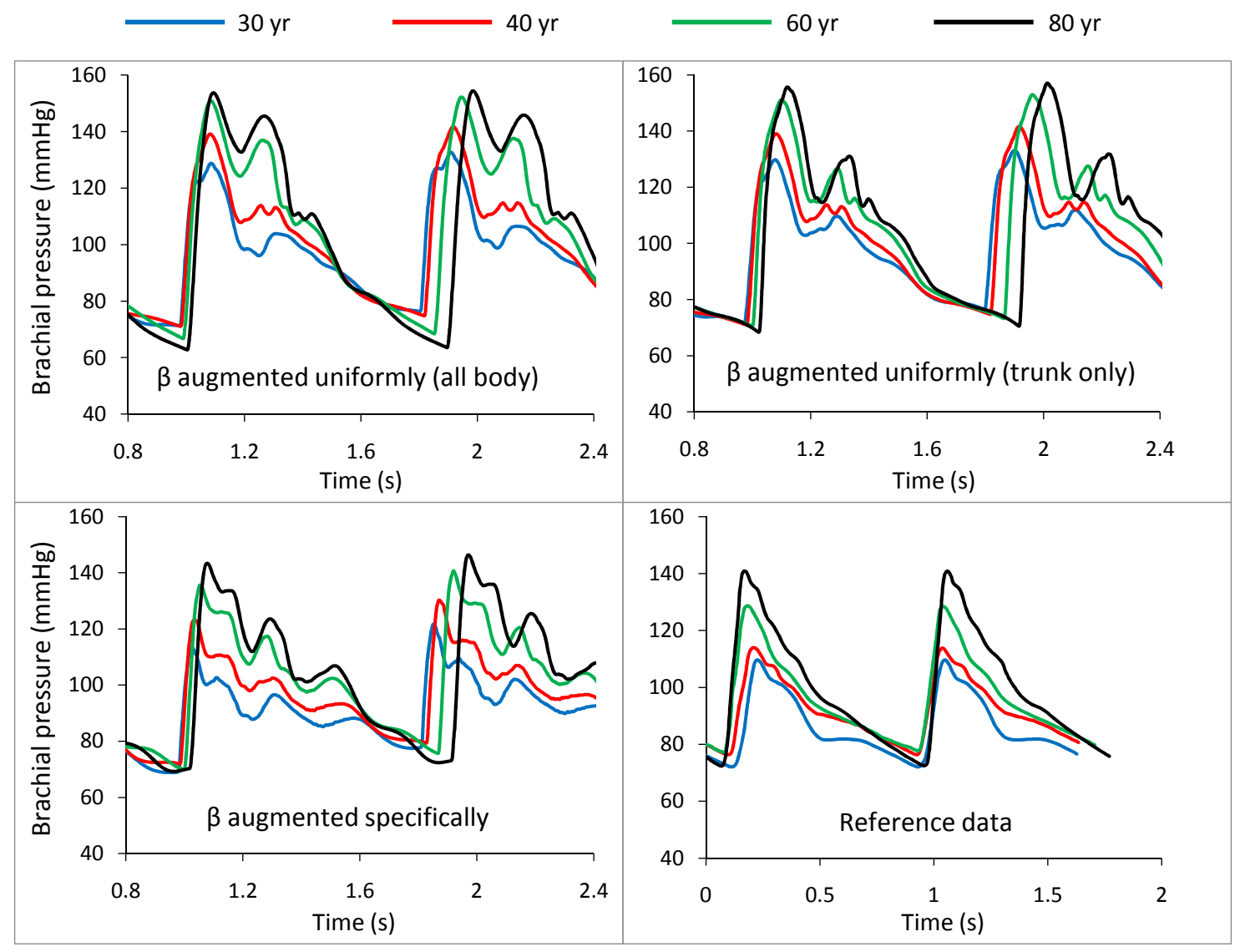

Figure 4.4: Pressure waveforms at brachial artery for different ages. Reference data are taken from [1].

with starting blood and tissue temperatures equal to $37^{\circ} \mathrm{C}$. Simulations are carried out for two different ages (30 and $80 \mathrm{yr}$ ). To quantify the body thermal response, the core temperature and averaged skin temperature are used. Figure 4.7 shows core temperature variation with time for different ages and modelling hypotheses. The case $80 \mathrm{yr}$ (only fluid modified) shows that the age-related flow changes do not affect the body heat transfer since the results are the same as the case $30 \mathrm{yr}$. Tissue property changes, such as volume and metabolic reductions, have a more significant role in the heat balance, as the core temperature drops consistently over time. This occurs because in such case the thermoregulatory system has not been modified and works correctly. A reduced capacity of 


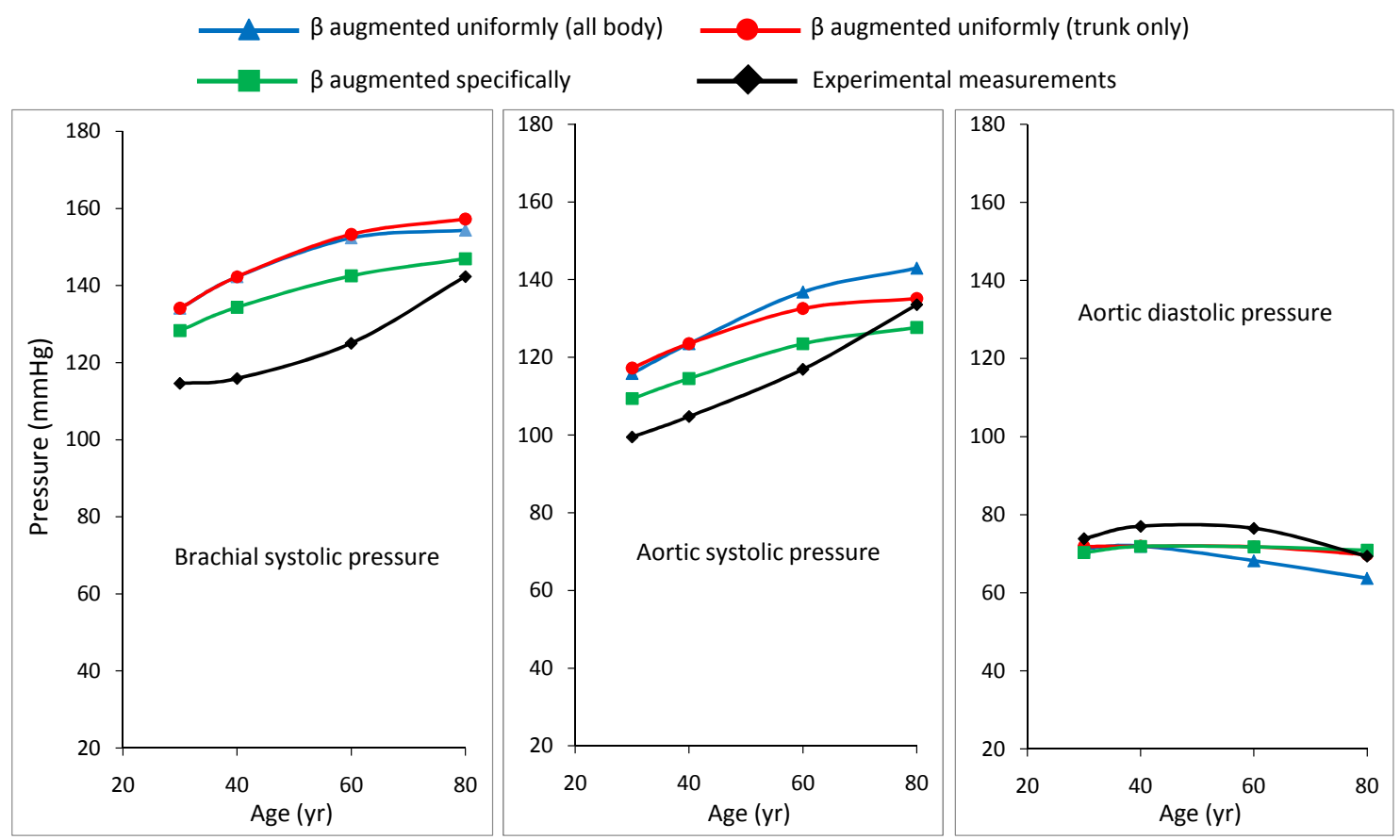

Figure 4.5: Systolic and diastolic pressure at ascending aorta and brachial artery for different ages. Experimental measurements are taken from [2,3].

regulating vasocutenous perfusion (case only fluid, tissues, vasodilation modified) hinders such fall in temperature. This effect is further augmented $\left(+0.2{ }^{\circ} \mathrm{C}\right)$ if also the sweating mechanism age-deterioration is accounted for. The study of the mean skin temperature evolution in time for different mechanisms of activation and age (see Figure 4.8) leads to similar conclusions.

\subsection{Conclusions}

In this work, a comprehensive methodology for modelling age effects on flow and heat transfer in the body is proposed. Each age modification introduced is derived from experimental data or from other computational methodologies. Different approaches for modelling age effects on specific model components are discussed and compared. The 


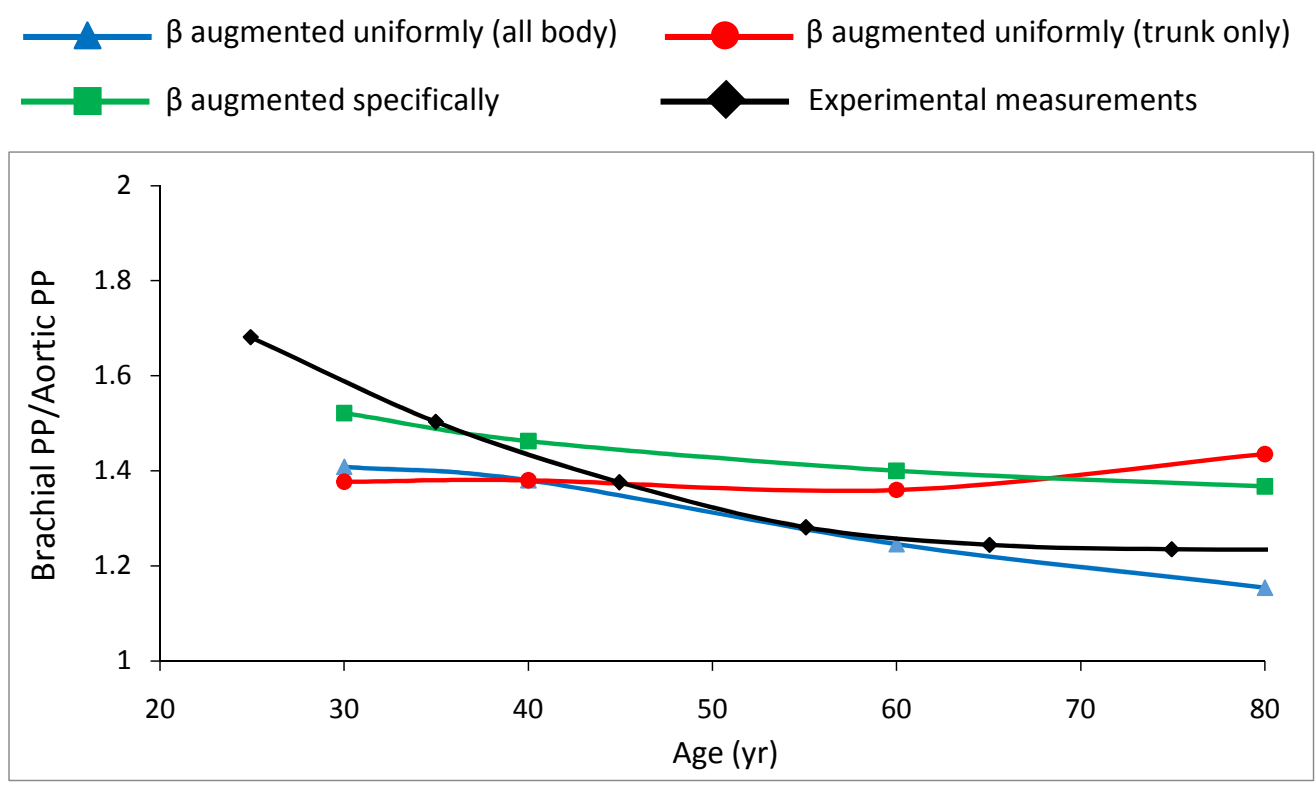

Figure 4.6: Pressure propagation indicators for different ages. Experimental measuements are taken from [3]

results obtained by applying such age-changes are also tested against literature data. Age modelling strategy for the arterial system is evaluated by analyzing pressure waveforms and other indicators at different sites of the arterial tree. In order to elucidate the agedependency of each body thermal component, the body model is exposed to hot stress conditions. This numerical study is characterized by employing body thermal indicators and tissue temperature distributions. Simulation results show that flow variations due to age do not have a significant effect on tissue body temperature distribution. It is also shown that, under thermal stress conditions, thermoregulatory system deterioration can profoundly affect the body thermal balance. 

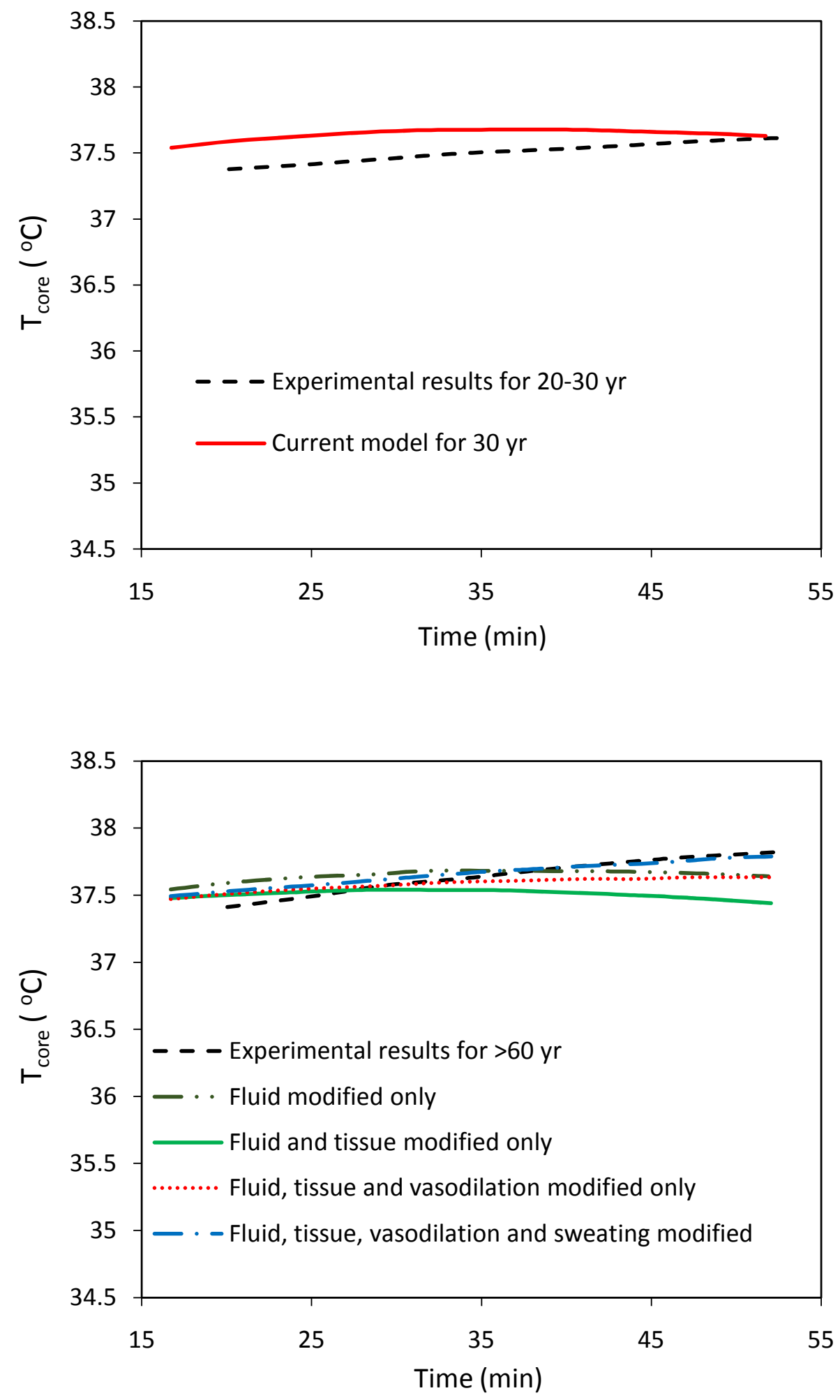

Figure 4.7: Core temperature evolution in time for different modelling assumptions. Literature results are taken from [4] (core temperature is assumed to be $37.3{ }^{\circ} \mathrm{C}$ at the beginning of the test). 

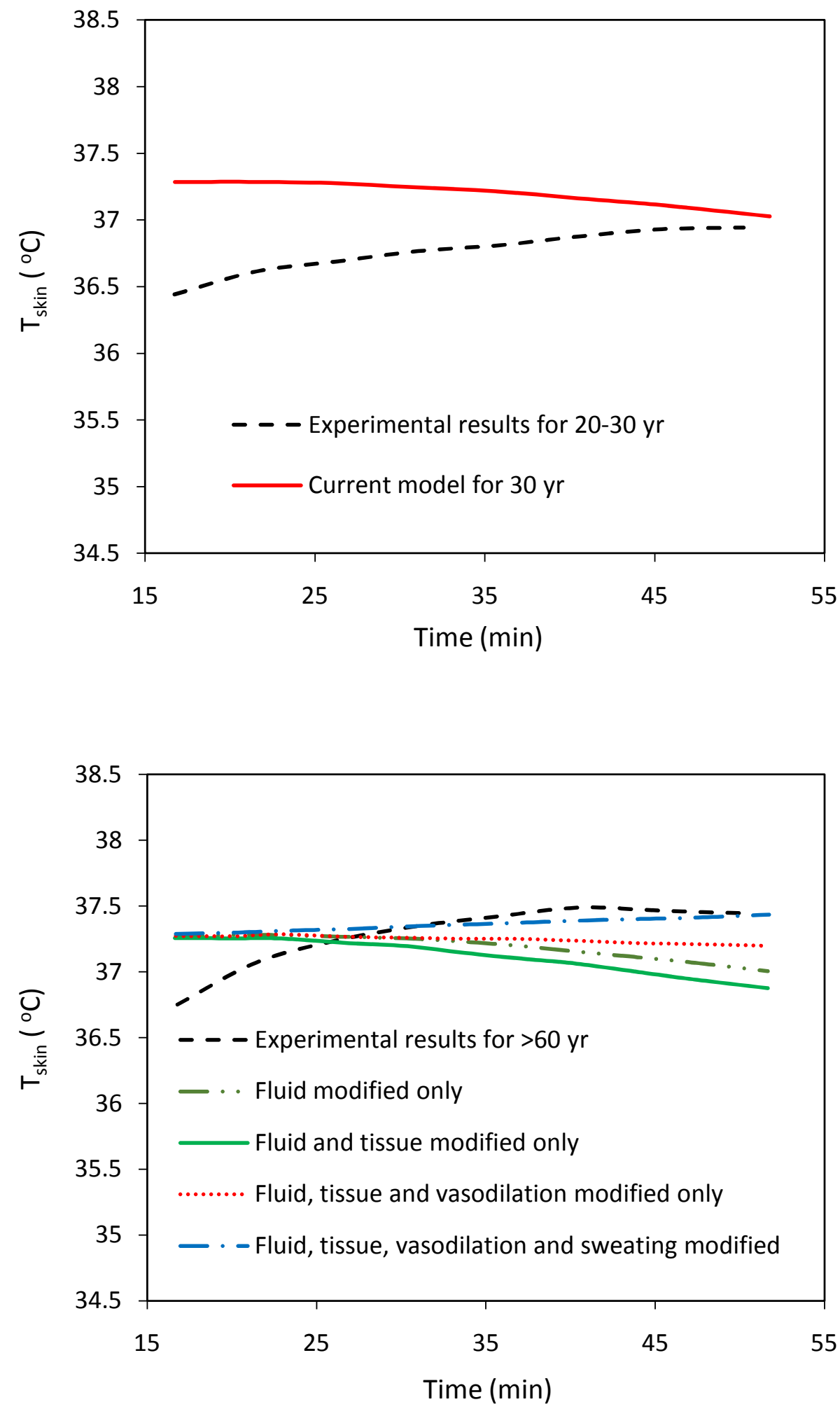

Figure 4.8: Mean skin temperature evolution in time for different modelling assumptions. Experimental results are taken from [5] (skin temperature is assumed to be $34.4{ }^{\circ} \mathrm{C}$ at the beginning of the test). 
Chapter 5

Conclusions and future works 


\subsection{Introduction}

In this chapter, we shall draw the key points of the current work and then followed by some recommendations for future.

\subsection{Conclusions}

A family of three novel semi-implicit locally conservative Galerkin methods (SILCG) has been developed for solving blood flow problem in a human arterial network. Based on Taylor expansion technique SILCG-TE, following to streamline upwind Petrov-Galerkin method SILCG-SUPG and finally backward in time and central in space approach SILCGBTCS. In all proposed methods, no matrix assembly is required and therefore they deal with an element matrix (i.e. $4 \times 4$ in this case). This avoids large matrices that often cost computer memory and slow the calculations. This allows us to use the traditional Newton method that requires evaluation of Jacobian matrix at every single iteration. The new approaches are not unconditionally stable due to the flux continuity over element edges is recalled from previous time step. Also, the boundary conditions are derived from the previous time step (characteristic system method for prescribing the boundaries). The numerical findings show that all proposed schemes are stable and the results are accurate against the reference data given by established explicit LCG. The computational cost per element is significantly lower especially when SILCG-BTCS is used. This is due to the simplified numerical procedure in comparison with SILCG-TE and SILCGSUPG. So, it is the fastest approach. Due to the lack of convection stabilisation terms, the method is more sensitive to increase in time steps with maximum value is limited to $\Delta t=0.1 \mathrm{~ms}$. Other SILCG methods can accept larger time steps up to $\Delta t=0.15 \mathrm{~ms}$. Also, the experience proves that Jacobian matrix calculations can be limited to only once every time step (not every iteration), so more computational cost can be saved.

All previous properties are applied to bioheat transfer problem in a human circulatory 
system. In chapter 3, all new approaches have been extended to solving energy equation. This is another task demonstrates the ability of the new solvers at dealing with different problems. The SILCG method in its three versions has been detailed and the results agree with data in the literature. Similar to flow, SILCG-BTCS is still the fastest and most sensitive one to time step changes for the same reasons mentioned previously (i.e. non presence of any stabilisation terms). Other techniques are stable beyond $\Delta t=0.1 \mathrm{~ms}$. Also, limiting Jacobian matrix solution to one factorization per time step is valid here as have been shown in the outcomes.

Finally, age-related effects on flow and bioheat balance have been modelled in chapter 4. The SILCG-TE is chosen to solve both flow and heat governing equations within a human body. The results have shown that age impacts pressure wave causing an obvious rise in pressure. This is because arteries stiffen with age and this enforces the heart to work harder to overcome the reflected waves. This is reflected in the input pressure wave and elastance that both rise with age. Also, age influence on temperature distribution in body tissues has been analyzed via numerical tests. All fluid, sweating and vasodilation mechanisms are included. The outcomes show that fluid alone has limited impact on the thermal distribution. Other mechanisms related to thermoregulatory system deterioration with age such as sweating and vasodilation have the major impact. The results agree with reference results for both skin and core temperatures, and previously for flow.

Overall, SILCG in its three versions provides an useful tool to solve the non-linear and linear governing equations. Also, it allows us to apply the classical Newton solver that is considered as "a powerful solver". Moreover, SILCG generates only an element matrix which gives the user flexibility to use a finer mesh (smaller element size). The results show that SILCG-BTCS is the best approach among the three techniques presented in this study in terms of computational time and the accuracy as demonstrated before. However, the other methods are able to cope with a larger time step. 


\subsection{Future investigations}

The proposed three schemes, SILCG-TE, SILCG-SUPG, and SILCG-BTCS have shown consistency for both flow and heat problems in a human body. They give a starting point for further investigation that may optimize the work shown here. Further research is required to make the proposed methods fully implicit and more computationally efficient. This could be done by finding special remedies to the fluxes between elements where the accuracy of LCG, in general, depends on the accuracy of these fluxes. This refers to both flow and heat problem solvers. Replacing the boundary conditions approach is also necessary to improve time step size. Those boundaries restrict time step because they are evaluated from previous time step. Further investigation of comprehensive models that account for the effect of the surrounding tissue contraction on the vessel wall will enhance the predictions. More realistic three dimensional solvers is another potential work for improving the accuracy. Although those solvers tend to be computationally expensive, they can be useful for specific regions like boundaries and for coupling with one dimensional solver.

The results have showed that SILCG-BTCS is the best approach among the methods presented in terms of speed and accuracy, and therefore it is recommended for at least circulation kind of problems. However, stability restriction may enforce us to use a smaller time step. In this case, the other two techniques may be chosen as they can go beyond $\mathrm{CFL}=1$. So, it is left to the user to chose what method to use as all proposed approaches are applicable to problems that original LCG is capable of solving. This includes, pure diffusion, convection-diffusion and Navier-Stokes that have been solved using the established explicit LCG, based on Taylor expansion stabilized method like SILCG-TE developed in this work but with semi-implicit time discretization. Moreover, SILC-SUPG and SILCGBTCS are completely new solvers and they can be also used for the above problems. This apparently gives variety of options to the user. In summary, the proposed approaches can be taken to all problems that established LCG is able to solve and considering the 
extension of proposed methods to different problems is recommended.

With respect to ageing, including more realistic elements will obviously enhance the results. This can be done by including the lumen diameter changes with ageing, i.e. through Eq. 4.2. This perhaps requires a further improvement of the empirical correlations that were used for creating Eq. (4.2). Another possible improvement is taking non-linear relation of the arterial stiffness with age to improve predictions. 


\section{Appendix A}

Here, the finite elements discrete procedure details are given. First, rewriting Eq. (2.10) as

$$
\frac{\mathbf{U}^{n+1}-\mathbf{U}^{n}}{\Delta t}=\left[\mathbf{S}-\frac{\partial \mathbf{F}}{\partial x}+\frac{\Delta t}{2} \mathbf{C S}-\frac{\Delta t}{2} \mathbf{C} \frac{\partial \mathbf{F}}{\partial x}-\frac{\Delta t}{2} \mathbf{H} \frac{\partial \mathbf{S}}{\partial x}+\frac{\Delta t}{2} \mathbf{H} \frac{\partial^{2} \mathbf{F}}{\partial x^{2}}\right]^{n+1} .
$$

Rearranging above equation gives

$$
\frac{\mathbf{U}^{n+1}-\mathbf{U}^{n}}{\Delta t}=\left[\left(1+\frac{\Delta t}{2} \mathbf{C}\right) \mathbf{S}-\left(1+\frac{\Delta t}{2} \mathbf{C}\right) \frac{\partial \mathbf{F}}{\partial x}+\frac{\Delta t}{2} \mathbf{H}\left(\frac{\partial^{2} \mathbf{F}}{\partial x^{2}}-\frac{\partial \mathbf{S}}{\partial x}\right)\right]^{n+1} .
$$

Let

$$
a=b=\left(1+\frac{\Delta t}{2} \mathbf{C}\right), \quad c=\frac{\Delta t}{2} \mathbf{H} .
$$

Now reproducing Eq. (2) reads

$$
\frac{\mathbf{U}^{n+1}-\mathbf{U}^{n}}{\Delta t}=\left[a \mathbf{S}-b \frac{\partial \mathbf{F}}{\partial x}+c \frac{\partial^{2} \mathbf{F}}{\partial x^{2}}-c \frac{\partial \mathbf{S}}{\partial x}\right]^{n+1} .
$$

Elements of the matrices $\mathbf{C}$ and $\mathbf{H}$ given in Eqs. (2.20) and (2.21) are averaged over the elements as discussed before. Now, applying linear test function to the above equation generates [107]

$$
\int_{\Omega}[\mathbf{N}]^{T}\left(\frac{\mathbf{U}^{n+1}-\mathbf{U}^{n}}{\Delta t}\right) d \Omega=\int_{\Omega}[\mathbf{N}]^{T}\left[a \mathbf{S}-b \frac{\partial \mathbf{F}}{\partial x}+c \frac{\partial^{2} \mathbf{F}}{\partial x^{2}}-c \frac{\partial \mathbf{S}}{\partial x}\right]^{n+1} d \Omega
$$

where $\Omega$ is the domain. The results of the left hand side of the previous equation is already given in Eq. (2.15 or lumped in Eq. (2.16). The right hand side procedure starts with first 
term as

$$
\begin{array}{r}
a \int_{\Omega}[\mathbf{N}]^{T}[\mathbf{N}]\{\mathbf{S}\}^{n+1} d \Omega=a \int_{\Omega}\left[\begin{array}{l}
N_{1} \\
N_{2}
\end{array}\right]\left[\begin{array}{ll}
N_{1} & N_{2}
\end{array}\right]\left\{\begin{array}{c}
S_{1} \\
S_{2}
\end{array}\right\}^{n+1} d \Omega \\
=a \frac{l_{e}}{6}\left[\begin{array}{ll}
2 & 1 \\
1 & 2
\end{array}\right]\left\{\begin{array}{c}
S_{1} \\
S_{2}
\end{array}\right\}^{n+1}=\left(1+\frac{\Delta t}{2} \mathbf{C}\right) \frac{l_{e}}{6}\left[\begin{array}{ll}
2 & 1 \\
1 & 2
\end{array}\right]\left\{\begin{array}{c}
S_{1} \\
S_{2}
\end{array}\right\}^{n+1},
\end{array}
$$

where $l_{e}$ is the element length. The system above needs to be expanded as there are two unknowns at each node and four unknowns per each element as discussed in subsection 2.2.2. The rest of Eq. (5) follows the usual way and applying Green's lemma as [107, 39]

$$
\begin{gathered}
-b \int_{\Omega}[\mathbf{N}]^{T}[\mathbf{N}] \frac{\partial \mathbf{F}^{n+1}}{\partial x} \quad d \Omega=b \int_{\Omega} \frac{[\partial \mathbf{N}]^{T}}{\partial x}[\mathbf{N}]\{\mathbf{F}\}^{n+1} d \Omega-b \int_{\Gamma}[\mathbf{N}]^{T}\{\mathbf{F}\}^{n+1} \mathbf{n} d \Gamma \\
=\int_{\Omega}\left[\begin{array}{c}
\frac{\partial N_{1}}{\partial x} \\
\frac{\partial N_{2}}{\partial x}
\end{array}\right]\left[\begin{array}{ll}
N_{1} & N_{2}
\end{array}\right]\left\{\begin{array}{c}
F_{1} \\
F_{2}
\end{array}\right\} \quad d \Omega=b \frac{1}{2}\left[\begin{array}{cc}
-1 & -1 \\
1 & 1
\end{array}\right]\left\{\begin{array}{c}
F_{1} \\
F_{2}
\end{array}\right\}{ }^{n+1} \\
=\left(1+\frac{\Delta t}{2} \mathbf{C}\right) \frac{l}{2}\left[\begin{array}{cc}
-1 & -1 \\
1 & 1
\end{array}\right]\left\{\begin{array}{c}
F_{1} \\
F_{2}
\end{array}\right\}^{n+1} .
\end{gathered}
$$

The last term of the above equation represent the boundaries (i.e. $\Gamma$ ) and it is used for enforcing the flux continuity between elements for LCG method as discussed in subsection 2.2.2. Thus, the interest is in the matrices here. Other terms are treated as

$$
\begin{aligned}
& c \int_{\Omega}[\mathbf{N}]^{T}[\mathbf{N}]{\frac{\partial^{2} \mathbf{F}^{n+1}}{\partial x^{2}}}^{n} d \Omega=-c \int_{\Omega} \frac{[\partial \mathbf{N}]^{T}}{\partial x} \frac{[\partial \mathbf{N}]}{\partial x}\{\mathbf{F}\}^{n+1} d \Omega \\
& =-c \int_{\Omega}\left[\begin{array}{c}
\frac{\partial N_{1}}{\partial x} \\
\frac{\partial N_{2}}{\partial x}
\end{array}\right]\left[\begin{array}{ll}
\frac{\partial N_{1}}{\partial x} & \frac{\partial N_{2}}{\partial x}
\end{array}\right]\left\{\begin{array}{c}
F_{1} \\
F_{2}
\end{array}\right\}^{n+1} d \Omega=-c \frac{1}{l_{e}}\left[\begin{array}{cc}
1 & -1 \\
-1 & 1
\end{array}\right]\left\{\begin{array}{c}
F_{1} \\
F_{2}
\end{array}\right\}^{n+1} \\
& =-\frac{\Delta t}{2} \mathbf{H} \frac{l}{l_{e}}\left[\begin{array}{cc}
1 & -1 \\
-1 & 1
\end{array}\right]\left\{\begin{array}{l}
F_{1} \\
F_{2}
\end{array}\right\}^{n+1} \text {, }
\end{aligned}
$$




$$
\begin{array}{r}
-c \int_{\Omega}[\mathbf{N}]^{T}[\mathbf{N}] \frac{\partial \mathbf{S}^{n+1}}{\partial x} d \Omega=c \int_{\Omega} \frac{[\partial \mathbf{N}]^{T}}{\partial x}[\mathbf{N}]\{\mathbf{S}\}^{n+1} d \Omega \\
=c \int_{\Omega}\left[\begin{array}{c}
\frac{\partial N_{1}}{\partial x} \\
\frac{\partial N_{2}}{\partial x}
\end{array}\right]\left[\begin{array}{ll}
N_{1} & N_{2}
\end{array}\right]\left\{\begin{array}{c}
S_{1} \\
S_{2}
\end{array}\right\}^{n+1} d \Omega=c \frac{1}{2}\left[\begin{array}{cc}
-1 & -1 \\
1 & 1
\end{array}\right]\left\{\begin{array}{c}
S_{1} \\
S_{2}
\end{array}\right\}^{n+1} \\
=\frac{\Delta t}{2} \mathbf{H} \frac{l}{2}\left[\begin{array}{cc}
-1 & -1 \\
1 & 1
\end{array}\right]\left\{\begin{array}{c}
S_{1} \\
S_{2}
\end{array}\right\}^{n+1}
\end{array}
$$

As mentioned before that above presentation need to be expanded to $4 \times 4$ matrices as there are four unknowns per each element as discussed in subsection 2.2.2. 


\section{Bibliography}

[1] Pagoulatou S. and Stergiopulos N. Evolution of aortic pressure during normal ageing: A model-based study. PLoS ONE, 12(7):e0182173, 2017.

[2] S. S. Franklin, W. Gustin, N. D. Wong, M. G. Larson, M. A. Weber, and W. B. et al. Kannel. Hemodynamic patterns of age-related changes in blood pressure. the framingham heart study. Circulation, 96:308-15, 1997.

[3] C. M. McEniery, Yasmin, B. McDonnell, M. Munnery, S. M. Wallace, and C. V. et al. Rowe. Central pressure: variability and impact of cardiovascular risk factors: the anglo-cardiff collaborative trial ii. Hypertens Dallas Tex 1979, 51:1476-82, 2008 .

[4] Hirata A., Nomura T., and Laakso I. Computational estimation of body temperature and sweating in the aged during passive heat exposure. International Journal of Thermal Sciences, 89:154-163, 2015.

[5] A. Dufour and V. Candas. Ageing and thermal responses during passive heat exposure: sweating and sensory aspects. Eur. J. Appl. Physiol., 100:19-26, 2007.

[6] A. Coccarelli, E. Boileau, D. Parthimos, and P. Nithiarasu. An advanced computational bioheat transfer model for a human body with an embedded systemic circulation. Biomechanics and Modeling in Mechanobiology, 15(5)(DOI: 10.1007/s10237-015-0751-4):1173-1190, 2016. 
[7] Maksuti E., Westerhof N., Westerhof B. E., Broome' M., and Stergiopulos N. Contribution of the arterial system and the heart to blood pressure during normal aging - a simulation study. PLoS ONE, 11(6):e0157493, 2016.

[8] G. Fu. A transient 3-D mathematical thermal model for the clothed human. Ph.D thesis, Kansas State University, 1995.

[9] D. Fiala. A computer model of human thermoregulation for a wide range of environmental conditions: The passive system. Journal of Applied Physiology, 87:1957-1972, 1999.

[10] H.M. Hasan, A. Coccarelli, and P. Nithiarasu. Novel semi-implicit, locally conservative galerkin (silcg) methods: Application to blood flow in a systemic circulation. Computer Methods in Applied Mechanics and Engineering, 332:217-233, 2018.

[11] A.R. Ghigoa, O. Delestreb, J.-M. Fullanaa, and P.-Y. Lagrée. Low-shapiro hydrostatic reconstruction technique for blood flow simulation in large arteries with varying geometrical and mechanical properties. Journal of Computational Physics, 331:108-136, 2017.

[12] K. Low, R. van Loon, I. Sazonov, R. L. T. Bevan, and P. Nithiarasu. An improved baseline model for a human arterial network to study the impact of aneurysms on pressure-flow waveforms. International Journal for Numerical Methods in Biomedical Engineering, 28:1224-1246, 2012.

[13] I. Sazonov, S.W. Khir, W.S. Hacham, E. Boileau, J.M. Carson, R. van Loon, C. Ferguson, and P. Nithiarasu. A novel method for non-invasively detecting the severity and location of aortic aneurysms. Biomechanics and modeling in mechanobiology, DOI 10.1007/s10237-017-0884-8, Feb 2017.

[14] A. Eken and M. Sahin. A parallel monolithic approach for fluid-structure interaction in a cerebral aneurysm. Computers and Fluids, 153:61-75, 2017. 
[15] A. Antonov, K. Kono, K. Greim-Kuczewski, J. E. Hippelheuser, A. Lauric, and A. M. Malek. Proximal stenosis is associated with rupture status in middle cerebral artery aneurysms. World Neurosurgery, 109:835-844, 2018.

[16] C. Vergara, D.L. Van, M. Quadrio, L. Formaggia, and M. Domanin. Large eddy simulations of blood dynamics in abdominal aortic aneurysms. Medical Engineering and Physics, 47:38-46, 2017.

[17] H. Liu, L. Lan, X. Leng, H. L. Ip, T.W.H. Leung, D. Wang, and K.S Wong. Impact of side branches on the computation of fractional flow in intracranial arterial stenosis using the computational fluid dynamics method. Journal of Stroke and Cerebrovascular Diseases, 27:44-52, 2018.

[18] A. Jonášová and J. Vimmr. Noninvasive assessment of carotid artery stenoses by the principle of multiscale modelling of non-newtonian blood flow in patientspecific models. Applied Mathematics and Computation, 319:598-616, 2018.

[19] E. Boileau and P. Nithiarasu. One-dimensional modelling of the coronary circulation. application to noninvasive quantification of fractional flow reserve (ffr). In J.M.R.S Tarves and R.M.N. Jorge, editors, Computational and experimental biomedical sciences: Methods and Applications, pages 137-156. Springer, 2013.

[20] Etienne Boileau, Sanjay Pant, Carl Roobottom, Igor Sazonov, Jingjing Deng, Xianghua Xie, and Perumal Nithiarasu. Estimating the accuracy of a reduced-order model for the calculation of fractional flow reserve (ffr). International Journal for Numerical Methods in Biomedical Engineering, 10.1002/cnm.2908:e2908.

[21] J. R. Womersley. An elastic tube theory of pulse transmission and oscillatory flow in mammalian arteries. Technical Report TR 56-614, WADC Technical Report, 1957. 
[22] A. Avolio. Multi-branched model of the human arterial system. Medical and Biological Engineering and Computing, 18(6):709-718, 1980.

[23] D.J. Brown. Input impedance and reflection coefficient in fractal-like models of asymmetrically branching compliant tubes. IEEE Transactions on Biomedical Engineering, 43(7):715-722, 1996.

[24] Y. Huo and G.S. Kassab. A hybrid one-dimensional/womersley model of pulsatile blood flow in the entire coronary arterial tree. American Journal of Physiology, 292:2623-2633, 2007.

[25] N. Westerhof, F. Bosman, C.J. De Vries, and A. Noordergraaf. Analog studies of the human systemic arterial tree. Journal of Biomechanics, 2(2):121-134, 1969.

[26] W.E. Bodley. The non-linearities of arterial blood flow. Physics in Medicine and Biology, 16(4):663-672, 1971.

[27] P.J. Reuderink, H.W. Hoogstraten, P. Sipkema, B. Hillen, and N. Westerhof. Linear and nonlinear one-dimensional models of pulse wave transmission at high womersley numbers. Journal of Biomechanics, 22(8-9):819-827, 1989.

[28] S. J. Sherwin, V. Franke, J. Peiró, and K. Parker. One-dimensional modelling of a vascular network in space-time variables. Journal of Engineering Mathematics, 47:217-250, 2003.

[29] J.K. Raines, M.Y. Jaffrin, and A.H. Shapiro. A computer simulation of arterial dynamics in the human leg. Journal of Biomechanics, 7(1):77-91, 1974.

[30] N. Stergiopulos, D.F. Young, and T.R. Rogge. Computer simulation of arterial flow with applications to arterial and aortic stenoses. Journal of Biomechanics, 25(12):1477-1488, 1992. 
[31] C. Sheng, S. Sarwal, K. Watts, and A. Marble. Computational simulation of blood flow in human systemic circulation incorporating an external force field. Medical and Biological Engineering and Computing, 33(1):8-17, 1995.

[32] B.W. Schaaf and P.H. Abbrecht. Digital computer simulation of human systemic arterial pulse wave transmission: A nonlinear model. Journal of Biomechanics, 5(4):345-364, 1972.

[33] S. A. Urquiza, P. J. Blanco, M. J. Vénere, and R. A. Feijóo. Multidimensional modelling for the carotid artery blood flow. Computer Methods in Applied Mechanics and Engineering, 195:4002-4017, 2006.

[34] J. Wan, B. Steele, S.A. Spicer, S. Strohband, G.R. Feijóo, T.J.R. Hughes, and C.A. Taylor. A one-dimensional finite element method for simulation-based medical planning for cardiovascular disease. Computer Methods in Biomechanics and Biomedical Engineering, 5(3):195 - 206, 2002.

[35] L. Formaggia, F. Nobile, and A. Quarteroni. A one-dimensional model for blood flow: application to vascular prosthesis. In I. Babuška and et al., editors, Mathematical Modeling and Numerical Simulation in Continuum Mechanics, volume 19 of Lecture Notes in Computational Science and Engineering, pages 137-153, 2002.

[36] Jason Carson and Raoul Van Loon. An implicit solver for 1d arterial network models. International Journal for Numerical Methods in Biomedical Engineering, 33(e2837), 2017.

[37] Christian Contarino, Eleuterio F. Toro, Gino I. Montecinos, Raul Borsche, and Jochen Kall. Junction-generalized riemann problem for stiff hyperbolic balance laws in networks: An implicit solver and ader schemes. Journal of Computational Physics, 315:409-433, 2016. 
[38] P. J. Blanco, S. M. Watanabe, E. A. Dari, M. A. R. F. Passos, and R. A. Feijóo. Blood flow distribution in an anatomically detailed arterial network model: criteria and algorithms. Biomech Model Mechanobiol, 13:1303-1330, 2014.

[39] J.P. Mynard and P. Nithiarasu. A 1D arterial blood flow model incorporating ventricular pressure, aortic valve and regional coronary flow using locally conservative Galerkin (LCG) method. Communications in Numerical Methods in Engineering, 24:367-417, 2008.

[40] E. Boileau, P. Nithiarasu, J.B. Blanco, L.O. Muller, F. E.E. Fossans, L.R. Helleviks, W.P. Doners, W. Huberts, M. Willemet, and J. Alastruey. A benchmark study of 1-d numerical schemes for arterial blood flow modelling. International Journal for Numerical Methods in Biomedical Engineering, e02732(DOI: 10.1002/cnm.2732), 2015 .

[41] A.N. Brooks and T.J.R. Hughes. Streamline upwind/Petrov-Galerkin formulation for convection dominated flows with particular emphasis on the incompressible navier stokes equation. Computer Methods in Applied Mechanics and Engineering, 32:199-259, 1982.

[42] S. S. Simakov and A. S. Kholodov. Computational study of oxygen concentration in human blood under low frequency disturbances. Mathematical Models and Computer Simulations, 1(DOI:10.1134/S2070048209020112):283-295, 2009.

[43] A. C. I. Malossi, P. J. Blanco, and S. Deparis. A two-level time step technique for the partitioned solution of one-dimensional arterial networks. Computer Methods in Applied Mechanics and Engineering, 237-240:212-226, 2012.

[44] P. J. Blanco, J. S. Leiva, R. A. Feijóo, and G. C. Buscaglia. Black-box decomposing approach for computational hemodynamic: One-dimensional model. Computer Methods in Applied Mechanics and Engineering, 200:1389-1405, 2011. 
[45] W. H. Press, S. A. Teukolsky, W. T. Vetterling, and B. P. Flannery. Numerical Recipes in Fortran 77: the art of scientific computing. Cambridge University Press, second-volume 1 of fortran numerical recipes edition, 1996-1997.

[46] S.V. Craenendonck, L. Lauriks, C. Vuye, and J. Kampen. A review of human thermal comfort experiments in controlled and semicontrolled environments. $R e$ newable and Sustainable Energy Reviews, 82:3365-3378, 2018.

[47] L. Zhu, L. Pang, and L.X. Xu. Simultaneous measurements of local tissue temperature and blood perfusion rate in the canine prostate during radio frequency thermal therapy. Biomechan Model Mechanobiol, 4:1-9, 2005.

[48] P.B. Wells, S. Thomsen, M.A. Jones, S. Baek, and J.D. Humphrey. Histological evidence for the role of mechanical stress in modulating thermal denaturation of collagen. Biomechanics and Modeling in Mechanobiology, 4:201-210, 2005.

[49] T.I. Zohdi. Modeling electrical power absorption and thermally-induced biological tissue damage. Biomechanics and Modeling in Mechanobiology, 13:115-121, 2014.

[50] Y. Yang, R. Yao, B. Li, H. Liu, and L. Jiang. A method of evaluating the accuracy of human body thermoregulation models. Building and Environment, 87:1-9, 2015.

[51] A.P. Gagge, J.A.J. Stolwijk, and J.D. Hardy. Comfort and thermal sensation and associated physiological responses at various ambient temperatures. Environmental Research, 1:1-20, 1967.

[52] J.A.J. Stolwijk and J.D. Hardy. Partitional calorimetric studies of responses of man to thermal transients. Journal of Applied Physiology, 21:967-977, 1966. 
[53] J.A.J. Stolwijk. A mathematical model of physiological temperature regulation in man. Technical Report NASA CR-1855, National Aeronautics and Space Administration, 1971.

[54] J.A.J. Stolwijk. Control of body temperature. In Handbook of Physiology-Reaction to Environmental Agents, pages 45-67. 1977.

[55] M. Al-Othmani, N. Ghaddar, and K. Ghali. A multi-segmented human bioheat model for transient and asymmetric radiative environments. International Journal of Heat and Mass Transfer, 51:5522-5533, 2008.

[56] D. Fiala, K.J. Lomas, and M. Stohrer. Computer prediction of human thermoregulatory and temperature response to a wide range of environmental conditions. International Journal of Biometeorology, 45:143-159, 2001.

[57] C. Huizenga, Z. Hui, and E. Arens. A model of human physiology and comfort for assessing complex thermal environments. Building and Environment, 36:691-699, 2001.

[58] A. Coccarelli and P. Nithiarasu. A robust finite element modelling approach to conjugate heat transfer in flexible elastic tubes and tube networks. Numerical Heat Transfer, Part A Applications, 67:513-530, 2015.

[59] S. S Hajat, R. S. Kovats, and K. Lachowycz. Heat-related and cold-related deaths in england and wales: who is at risk? Occup Environ Med, 64:93-100, 2007.

[60] Kowald A., Dawson M., and Kirkwood T. B. L. Mitochondrial mutations and ageing: Can mitochondrial deletion mutants accumulate via a size based replication advantage? Journal of Theoretical Biology, 340:111-118, 2014.

[61] Miquel J., Economos A. C., Fleming J., and Johnson J. E. Jr. Mitochondrial role in cell aging. Experimental Gerontology, 15:575-591, 1980. 
[62] Harman D. Free radical theory of aging: consequences of mitochondrial aging. Age, 6:86-94, 1983.

[63] Richter C. Do mitochondrial dna fragments promote cancer and aging? FEBS Letters, 241:1-5, 1988.

[64] Linnane A. W., Marzuki S., Ozawa T., and Tanaka M. Mitochondrial dna mutations as an important contributor to ageing and degenerative diseases. Lancet, 1:642645, 1989.

[65] H. Bader. Dependance of wall stress in the human phoracic aorta on age and pressure. Circulation Research, 20:354-361, 1967.

[66] A. Benetos, S. Laurent, and et al. Arterial alterations with aging and high blood pressure. a noninvasive study of carotid and femoral arteries. Arteriosclerosis and Thrombosis, 13 (1):90-97, 1993.

[67] P. Segers, E. R. Rietzschel, M. L. De Buyzere, S. J. Vermeersch, D. De Bacquer, L. M. Van Bortel, and et al. Noninvasive (input) impedance, pulse wave velocity, and wave reflection in healthy middle-aged men and women. Hypertension, 49:1248-55, 2007.

[68] B. H. Lorell and B. A. Carabello. Left ventricular hypertrophy: pathogenesis, detection, and prognosis. Circulation, 102:470-9, 2000.

[69] A. U. Ferrari, A. Radaelli, and M. Centola. Invited review: Aging and the cardiovascular system. J Appl Physiol, 95:2591-7, 2003.

[70] M. F. O’Rourke and J. Hashimoto. Mechanical factors in arterial aging: A clinical perspective. Journal of the American College of Cardiology, 50:1-13, 2007. 
[71] G. F. Mitchell, S.-J. Hwang, R. S. Vasan, M. G. Larson, M. J. Pencina, and N. M. et al. Hamburg. Arterial stiffness and cardiovascular events. Circulation, 121:505$11,2010$.

[72] A. S. Jackson and M. L. Pollock. Generalized equations for predicting body density of men. Br. J. Nufr., 40:497-504, 1978.

[73] A. J. Cruz-Jentoft, J. P. Baeyens, J. M. Bauer, Y. Boirie, T. Cederholm, Francesco Landi, F. C. Martin, J.-P. Michel, Y. Rolland, and Schneider S. M. et al. Sarcopenia: European consensus on definition and diagnosis. Age and Ageing, 39:412-423, 2010.

[74] M. F. Kuczmarski, R. J. Kuczmarski, and M. Najjar. Descriptive anthropometric reference data for older americans. Journal of THE AMERICAN DIETETIC ASSOCIATION, 100:59-66, 2000.

[75] N. Emaus, G. K. R. Berntsen, R. Joakimsen, and V. Fonnebø. Longitudinal changes in forearm bone mineral density in women and men aged 45-84 years: The troms $\varnothing$ study, a population-based study. American Journal of Epidemiology, 163(5):441449, 2006.

[76] M. J. Silva and L. J. Gibson. Modeling the mechanical behavior of vertebral trabecular bone: Effects of age-related changes in microstructure. Bone, 21(2):191-199, 1997.

[77] B. A. Christiansen, D. L. Kopperdahl, D. P. Kiel, T. M. Keaveny, and M. L. Bouxsein. Mechanical contributions of the cortical and trabecular compartments contribute to differences in age-related changes in vertebral body strength in men and women assessed by qct-based finite element analysis. JBMR, 26(5):974-983, 2011. 
[78] Chen H., Zhou X., Fujita H., Onozuka M., 3, and Kubo K.-Y. Age-related changes in trabecular and cortical bone microstructure. International Journal of Endocrinology, 2013:Article ID 213234, 9 pages, 2013.

[79] C. Ruggiero, E. J. Metter, V. Melenovsky, A. Cherubini, S. S. Najjar, A. Ble, U. Senin, D. L. Longo, and A. Ferrucci. High basal metabolic rate is a risk factor for mortality: The baltimore longitudinal study of aging. The Journals of Gerontology: Series A, 63A(7):698-706, 2008.

[80] E. T. Poehlman, E. M. Berke, J. R. Joseph, A. W. Gardner, S. M. Katzman-Rooks, and M. I. Goran. Influence of aerobic capacity, body composition, and thyroid hormones age-related decline in resting metabolic rate. Metabolism, 41:pp 915921, 1992.

[81] Novieto D. T. Adapting a human thermoregulation model for predicting the thermal response of older persons. PhD thesis, Montfort University, 2013.

[82] C. J. K. Henry. Basal metabolic rate studies in humans: measurement and development of new equations. Public Health Nutrition, 8(7A):1133-1152, 2005.

[83] W. L. Kenney and T. A. Munce. Physiology of aging invited review: Aging and human temperature regulation. J Appl Physiol, 95:2598-2603, 2003.

[84] C. Smith. A transient, Three Dimensional Model of the Human Thermal System. $\mathrm{PhD}$ thesis, Kansas State University, 1991.

[85] M. Salloum, N. Ghaddar, and K. Ghali. A new transient bioheat model of the human body and its integration to clothing model. International Journal of Thermal Sciences, 46:371-384, 2007. 
[86] M.S. Ferreira and J.I. Yanagihara. A transient three-dimensional heat transfer model of the human body. International Communications in Heat and Mass Transfer, 36:718-724, 2009.

[87] W. Karaki, N. Ghaddar, K. Ghali, K. Kalev, I. Holmer, and L.L. Vanguard. Human thermal response with improved ava modeling of the digits. Int J Therm Sci, 67:41$52,2013$.

[88] M. Rida, W. Karaki, N. Ghaddar, K. Ghali, and J. Hoballah. A new mathematical model to simulate ava cold-induced vasodilation reaction to local cooling. Int. J. Biometeorol., 58:1905-1918, 2014.

[89] J. A. Taylor, G. A. Hand, D. G. Johnson, and D. R. Seals. Sympathoadrenalcirculatory regulation of arterial pressure during orthostatic stress in young and older men. Am. J. Physiol., 263:R1147-R1155, 1992.

[90] G. Paolisso, A. Gambardella, Balbi V., S. Ammendola, A. D’Amore, and M. Varricchio. Body composition, body fat distribution, and resting metabolic rate in healthy centenarians. Am J Clin Nutr, 62 (4):746-50, 1995.

[91] C. T. Minson, S. L. Wladkowski, A. F. Cardell, J. A. Pawelczyk, and W. L. Kenney. Age alters the cardiovascular response to direct passive heating. J. Appl. Physiol., 84 (4):1323-1332, 1998.

[92] R.W. Ogden. Non-linear Elastic Deformations. Ellis Horwood, Limited (reprinted by Dover, 1997), Chichester, England, 1984.

[93] Rida M., Ghaddar N., Ghali K., and Hoballah J. Elderly bioheat modeling: changes in physiology, thermoregulation, and blood flow circulation. Int. J. Biometeorol., 58:1825-43, 2014. 
[94] A. Guala, C. Camporeale, and L. Ridolfi. Compensatory effect between aortic stiffening and remodelling during ageing. PLOS ONE, 10(10):e0139211, 2015.

[95] M. A. Zulliger and N. Stergiopulos. Structural strain energy function applied to the ageing of the human aorta. Journal of Biomechanics, 40(14):3061-9, 2007.

[96] J.D. Hardy and J.A.J. Stolwijk. Partitional exposures calorimetric studies of man during to thermal transients. Journal of Applied Physiology, 21:1799-1806, 1966.

[97] P. Nithiarasu. A simple locally conservative galerkin (LCG) finite-element method for transient conservation equations. Numerical Heat Transfer Part B - Fundamentals, 46(4):357-370, 2004.

[98] C. G. Thomas, P. Nithiarasu, and R. L. T. Bevan. The locally conservative galerkin (lcg) method for solving the incompressible navier-stokes equations. International Journal for Numerical Methods in Fluids, 57:1771-1792, 2008.

[99] C. G. Thomas and P. Nithiarasu. An element-wise, locally conservative galerkin (lcg) method for solving diffusion and convection-diffusion problems. International Journal for Numerical Methods in Engineering, 73:642-664, 2008.

[100] R.L.T. Bevan, P. Nithiarasu, R. Van Loon, I. Sazonov, H. Luckraz, and A. Garnham. Application of a locally conservative Galerkin (LCG) method for modelling blood flow through a patient-specific carotid bifurcation. International Journal for Numerical Methods in Fluids, 64:1274-1295, 2010.

[101] N. Zhang, Z. Huang, and J. Y. School. Locally conservative galerkin and finite volume methods for two-phase flow in porous media. J. Comput. Phys., 254:39$51,2013$. 
[102] S.J. Sherwin, V. Franke, J. Peiró, and K. Parker. One-dimensional modelling of a vascular network in space-time variables. Journal of Engineering Mathematics, 47(3):217-250, 2003.

[103] L. Formaggia, F. Nobile, and A. Quarteroni. A one dimensional model for blood flow: Application to vascular prosthesis. In Babuska I., Miyoshi T., and Ciarlet P.G., editors, Mathematical Modeling and Numerical Simulation in Continuum Mechanics, Lecture Notes in Computational Science and Engineering. SpringerVerlag, Berlin, 2002.

[104] M.S. Olufsen, C.S. Peskin, W.Y. Kim, E.M. Pedersen, A. Nadim, and J. Larsen. Numerical simulation and experimental validation of blood flow in arteries with structured-tree outflow conditions. Annals of Biomedical Engineering, 28:12811299, 2000.

[105] S. Acosta, C. Puelz, B. Riviere, D. J. Pennya, and C. G. Rusin. Numerical method of characteristics for one-dimensional blood flow. J. Comput. Phys., 294:96-109, 2015 .

[106] P. Nithiarasu, R.W. Lewis, and K.N. Seetharamu. Fundamentas of the finite element method for heat, mass and fluid flow. Wiley, second edition, 2016.

[107] R.W. Lewis, P. Nithiarasu, and K.N. Seetharamu. Fundamentals of the finite element method for heat and fluid flow. John Wiley and Sons, 2004.

[108] O. C. Zienkiewicz, R. L. Taylor, and P. Nithiarasu. The Finite Element Method for Fluid Dynamics. Elsevier, seventh edition, 2014.

[109] N.M. Abbasi. Solving the advection pde in explicit ftcs, lax, implicit ftcs and cranknicolson methods for constant and varying speed. accuracy, stability and software animation. MSc project, Mechanical engineering department University of California, Irvine. 
[110] O.C. Zienkiewicz, R.L. Taylor, and D.D. Fox. The finite element method for solid and structural mechanics. Elsevier, seventh edition, 2014.

[111] R. H. W. Hoppea and C. Linsenmann. An adaptive newton continuation strategy for the fully implicit finite element immersed boundary method. J. Comput. Phys., 231:4676-4693, 2012.

[112] C. D. Meyer. Matrix analysis and applied linear algebra. SIAM, 2000.

[113] S.J. Sherwin, L. Formaggia, J. Peiró, and V. Franke. Computational modelling of $1 \mathrm{D}$ blood flow with variable mechanical properties and its application to the simulation of wave propagation in the human arterial system. International Journal for Numerical Methods in Fluids, 43(6-7):673-700, 2003.

[114] H. Pennes. Analysis of tissue and arterial blood temperatures in the resting human forearm. Journal of applied physiology, 1:91-122, 1948.

[115] S. J. Sherwin, L. Formaggia, J. Peiró, and V. Franke. Computational modelling of $1 \mathrm{D}$ blood flow with variable mechanical properties and its application to the simulation of wave propagation in the human arterial system. International Journal for Numerical Methods in Fluids, 43(6-7):673-700, 2003. b.

[116] R. Holopainen. A human thermal model for improved thermal comfort. $\mathrm{PhD}$ thesis, VTT, Technical Research Centre of Finland, 2012.

[117] Refrigerating American Society of Heating and Air-Conditioning Engineers. Physiological principles and thermal comfort, 1993.

[118] A. Shitzer and R.C. Eberhart. Heat transfer in medicine and biology-analysis and applications. Plenum Press, New York, USA., 1985. 
[119] Pries A. R., Mulvany M. J., and Bakker E. N. T. P. Mbec special issue on microcirculation "engineering principles of vascular networks". Med. Biol. Eng. Comput., 46:407-409, 2008.

[120] B.W. Schaaf and P.H. Abbrecht. Digital computer simulation of human systemic arterial pulse wave transmission: a nonlinear model. Journal of Biomechanics, 5(4):345-364, 1972.

[121] Mynard J. P. and Nithiarasu P. A 1D arterial blood flow model incorporating ventricular pressure, aortic valve and regional coronary flow using the locally conservative Galerkin (LCG) method. Communications in Numerical Methods in Engineering, 24:367-417, 2008.

[122] R. Katori. Normal cardiac output in relation to age and body size. Tohoku J Exp Med, 128:377-87, 1979.

[123] E. G. Lakatta. Changes in cardiovascular function with aging. European Heart Journal, 11:22-29, 1990.

[124] Suga H., Sagawa K., and Shoukas A. A. Load independence of the instantaneous pressure-volume ratio of the canine left ventricle and effects of epinephrine and heart rate on the ratio. Circ. Res., 32:314-322, 1971.

[125] K. Sagawa. The end-systolic pressure-volume relation of the ventricle: definition, modifications and clinical use. Circulation, 63:1223-7, 1981.

[126] Stergiopulos N., Meister J. J., and Westerhof N. Determinants of stroke volume and systolic and diastolic aortic pressure. Am. J. Physiol., 270:H2050-H2059, 1996.

[127] L. Formaggia, D. Lamponi, M. Tuveri, and A. Veneziani. Numerical modeling of $1 \mathrm{~d}$ arterial networks coupled with a lumped parameters description of the heart. 
Computer Methods in Biomechanics and Biomedical Engineering, 9(5):273-288, 2006.

[128] T. Arts, P. Bovendeerd, F. W. Prinzen, and R. S. Reneman. Relation between left ventricular cavity pressure and volume and systolic fiber stress and strain in the wall. Biophys. J., 59:93-102, 1991.

[129] P. Bovendeerd, P. Borsje, T. Arts, and F. van De Vosse. Dependence of intramyocardial pressure and coronary flow on ventricular loading and contractility: a model study. Annals of Biomedical Engineering, 34 (12):1833-1845, 2006.

[130] C .M. McEniery, Yasmin, I. R. Hall, A. Qasem, I. B. Wilkinson, and J. R. et al. Cockcroft. Normal vascular aging: differential effects on wave reflection and aortic pulse wave velocity: the anglo-cardiff collaborative trial (acct). J Am Coll Cardiol, 46:1753-60, 2005.

[131] J. S. Petrofsky, H. J. Suh, S. Gunda, M. Prowse, and J. Batt. Interrelationships between body fat and skin blood flow and the current required for electrical stimulation of human muscle. Medical Engineering \& Physics, 30:931-936, 2008.

[132] H. Kanehisa, M. Miyatani, K. Azuma, S. Kuno, and T. Fukunaga. Influences of age and sex on abdominal muscle and subcutaneous fat thickness. Eur J Appl Physiol, 91:534-537, 2004.

[133] A. C. Looker, H. W. Wahner, W. L. Dunn, M. S. Calvo, T. B. Harris, S. P. Heyse, C. C. Johnston Jr, and R. Lindsay. Updated data on proximal femur bone mineral levels of us adults. Osteoporos Int, 8:468-489, 1998.

[134] K. E. Walker, T. Baldini, and B. G. Lindeque. Thermal conductivity of human bone in cryoprobe freezing as related to density. Orthopedics, 40(2):90-94, 2017. 
[135] C. Ogden, C. D. Fryar, M. D. Carroll, and K. M. Flegal. Mean bodyweight, height, and bodymassindex, united states 1960-2002. U.S. Department Of Health And Human Services, 347:1-18, 2004.

[136] I. P. Herman. Physics of the human body. Springer, second edition, 2016.

[137] D.M. Kerslake. The Stress of Hot Environments. Cambridge: University Press, 1972.

[138] G. S. Anderson, G. S. Meneilly, and I. B. Mekjavic. Passive temperature lability in the elderly. Eur J Appl Physiol, 73:278-286, 1996.

[139] L. A. Holowatz and Kenney W. L. Peripheral mechanisms of thermoregulatory control of skin blood flow in aged humans. J Appl Physiol, 109:1538-1544, 2010.

[140] Y. Tochihara, T. Ohnaka, Y. Nagal, T. Tokuda, and Kawashima Y. Physiological responses adnd thermal sensations of the elderly in cold and hot environments. $J$. therm. Biol., 18 (No. 5/6):355-361, 1993.

[141] D. I. Sessler. Temperature monitoring and perioperative thermoregulation. Anesthesiology, 109:318-38, 2008. 